\title{
Dynamical resource theory of quantum coherence
}

\author{
Gaurav Saxena $\odot^{*}$ \\ Department of Physics and Astronomy, Institute for Quantum Science and Technology, University of Calgary, AB, Canada T2N 1N4 \\ Eric Chitambar $\odot^{\dagger}$ \\ Department of Electrical and Computer Engineering, Coordinated Science Laboratory, \\ University of Illinois at Urbana-Champaign, Urbana, Illinois 61801, USA \\ Gilad Gour \\ Department of Mathematics and Statistics, Institute for Quantum Science and Technology, University of Calgary, AB, Canada T2N 1N4
}

(Received 17 October 2019; accepted 13 May 2020; published 8 June 2020)

\begin{abstract}
Decoherence is all around us. Every quantum system that interacts with the environment is doomed to decohere. While preserving quantum coherence is a major challenge faced in quantum technologies, the potential benefits for information processing are very promising since coherence can lead to various operational advantages, such as in quantum algorithms. Hence, much work has been devoted in recent years to quantify the coherence present in a system. In the present paper, we formulate the quantum resource theory of dynamical coherence. The underlying physical principle we follow is that the free dynamical objects are those that neither store nor output coherence. This leads us to identify classical channels as the free elements in this theory. Consequently, even the quantum identity channel is not free as all physical systems undergo decoherence and hence, the preservation of coherence should be considered a resource. The maximally coherent channel is then the quantum Fourier transform because of its abillity to preserve entanglement and generate maximal coherence from nothing. In our work, we introduce four different types of free superchannels (analogous to MIO, DIO, IO, and SIO) and discuss in detail two of them, namely, dephasing-covariant incoherent superchannels (DISC), maximally incoherent superchannels (MISC). The latter consists of all superchannels that do not generate non-classical channels from classical ones. We quantify dynamical coherence using channel-divergence-based monotones for MISC and DISC. We show that some of these monotones have operational interpretations as the exact, the approximate, and the liberal coherence cost of a quantum channel. Moreover, we prove that the liberal asymptotic cost of a channel is equal to a new type of regularized relative entropy. Finally, we show that the conversion distance between two channels under MISC and DISC can be computed using a semidefinite program (SDP).
\end{abstract}

DOI: 10.1103/PhysRevResearch.2.023298

\section{INTRODUCTION}

All physical systems undergo decoherence. It is an irreversible process, and it can be viewed as the reduction of a general quantum state to an incoherent mixed state due to coupling with the environment [1-3]. Mathematically, decoherence is represented as the vanishing of the off-diagonal terms of a density matrix. It is because of decoherence that we do not observe quantum mechanical behavior in everyday macroscopic objects, and in the context of quantum informa-

\footnotetext{
*gaurav.saxena1@ucalgary.ca

$\dagger$ echitamb@illinois.edu

${ }^{\ddagger}$ gour@ucalgary.ca
}

Published by the American Physical Society under the terms of the Creative Commons Attribution 4.0 International license. Further distribution of this work must maintain attribution to the author(s) and the published article's title, journal citation, and DOI. tion, it can be viewed as the loss of information from a system into the environment [4].

During the past two decades, interest in quantum information science has shifted towards using quantum mechanical phenomena (like entanglement, nonlocality, coherence, etc.) as resources to achieve something that is otherwise not possible through classical physics (e.g., quantum teleportation) [5-14]. Quantum resource theories (QRTs) use this resourcetheoretic approach to exploit the operational advantage of such phenomena and to assess their resource character systematically [15]. The preservation of quantum coherence is crucial for building quantum information devices, since the loss of quantum superposition due to decoherence negates any nonclassical effect in a quantum system $[1,16,17]$. Hence, from a technological perspective, there is increasing interest in developing a resource theory of coherence [15]. In addition, the resource-theoretic study of quantum coherence can provide new operational and quantitative insights into the differences between classical and quantum physics. Some other examples of quantum resource theories include the 
QRT of entanglement, thermodynamics, magic states, Bell nonlocality, etc.

Most quantum resource theories are governed by the constraints arising from physical or practical settings. These constraints then lead to the operations that can be freely performed. For instance, in the static resource theory of quantum entanglement, for any two spatially separated but possibly entangled systems, the spatial separation puts the restriction that only local operations along with classical communications (LOCC) can be performed [5,6,18-20]. Given this restriction, only separable states can be generated using LOCC, which makes them the free states of the theory. But unlike entanglement, whose free states (i.e., separable states) are determined from the a priori fixed set of operations (i.e., LOCC), coherence theory typically begins by fixing a set of free states. In this case, the free states are the physically motivated objects, and the free operations are not unique, only being required to satisfy the basic golden rule of a QRT, that the free operations should be completely resource nongenerating (CRNG) [15].

In the static (or state-based) resource theory of quantum coherence there is a fixed basis, the so-called classical or incoherent basis, and the set of density matrices that are diagonal in this basis form the free states of the theory. Such states are called incoherent states. The free operations are then some set of quantum channels that map the set of incoherent states to itself. The most well-studied classes of free operations are the maximally incoherent operations (MIO), the incoherent operations (IO), the dephasing-covariant incoherent operations (DIO), and the strictly incoherent operations (SIO) [21-26]. However, all of these operations cost coherence to be physically implemented in the sense that they do not always admit a free dilation [23-26]. Therefore, it can be questioned in what sense these operations are truly "free" [23]. However, as argued in Ref. [27], detecting the presence of resource in a given state should be possible using the free operations, and often this detection requires the consumption of resource. If such a detection is not possible, then both resource and nonresource states are equally useful (or useless) to the experimenter since the two cannot be distinguished, thus begging the question in what sense the former is truly a resource. In general then, having free operations with a nonzero resource cost can still lead to an insightful static resource theory. Indeed, even though $\mathrm{MIO} / \mathrm{DIO} / \mathrm{IO} / \mathrm{SIO}$ consume coherence in their implementation, they are still useful for comparing the coherence in two different states based on their convertibility using the given operations. A large amount of fruitful work has been devoted to developing the theory of static coherence under these operations [28].

A consequence of these observations is that the principles for assessing the resourcefulness of quantum states should not necessarily be applied when assessing the resourcefulness of quantum operations. In particular, the well-known approaches to quantifying the resourcefulness of a quantum channel in terms of its resource cost $[29,30]$ or its resource-generating power [31-35] can fall short of fully characterizing its utility in a resource theory. The ability for a channel to generate "resource detectability" is typically something not captured by its resource cost or resource-generation power [27]. Restricting to the resource theory of coherence, a POVM $\left\{P_{m}\right\}_{m}$ can

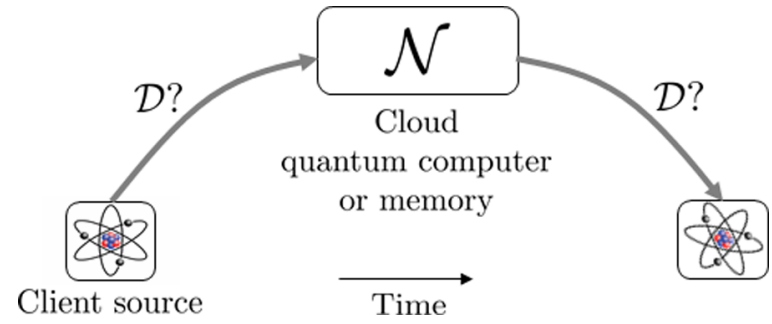

FIG. 1. A cloud quantum computer offers no computational resource if the upload and download channels are completely dephasing $(\mathcal{D})$. Channels having the form $\mathcal{D} \circ \mathcal{N} \circ \mathcal{D}$ are thus identified as free dynamical objects in the resource theory studied here.

detect the coherence in a state $\rho$ if $\operatorname{Tr}\left[\Pi_{m} \rho\right] \neq \operatorname{Tr}\left[\Pi_{m} \mathcal{D}(\rho)\right]$ for some outcome $m$, where $\mathcal{D}(\rho)=\sum_{i=1}^{d}|i\rangle\langle i|\rho| i\rangle\langle i|$ is the completely dephasing map in the incoherent basis $\{|i\rangle\}_{i=1}^{d}$. Since a POVM $\left\{P_{m}\right\}_{m}$ is unable to detect coherence in some state if (and only if) it is incoherent, i.e., $\mathcal{D}\left(P_{m}\right)=P_{m}$ for all $m$, a channel $\mathcal{N}$ fails to generate a detection of coherence if its dual maps any incoherent POVM to an incoherent POVM:

$$
\mathcal{N}^{\dagger} \circ \mathcal{D}\left(P_{m}\right)=\mathcal{D} \circ \mathcal{N}^{\dagger} \circ \mathcal{D}\left(P_{m}\right) \quad \forall\left\{P_{m}\right\}_{m} .
$$

Maps satisfying Eq. (1) have been called detection incoherent in Ref. [27] and nonactivating in Ref. [36], and any map not of this form is a dynamical resource from the coherencedetection perspective. It is not difficult to find channels that satisfy Eq. (1) while having a nonzero coherence cost and coherence-generating power (for instance, consider any replacement channel that outputs a coherent state for any input). A full resource theory can then be worked out on the level of channels in which maps having the form of Eq. (1) are free and the allowed operations are certain superchannels that act invariantly on the set of detection incoherent channels [27].

In this paper, we identify another coherence property of quantum channels that is not captured by coherence cost, coherence-generating power, or coherence detection. We are motivated by the interpretation of a quantum channel as a quantum memory that transmits quantum information from one point in spacetime to another [37-39]. As a concrete pragmatic scenario, we consider a cloud quantum computer in which a client uploads and downloads quantum information to a quantum computing processor and memory (see Fig. 1). Ideally, both the upload and download links are noiseless, and if $\rho$ is the quantum state sent to the cloud computer to perform operation $\mathcal{N}$, the state returned to the client will be $\mathcal{N}(\rho)$. However, in practice the channels connecting client to cloud will be noisy. In the extreme cases, a completely dephasing upload channel has the form $\mathcal{N}=\mathcal{N} \circ \mathcal{D}$ and can be interpreted as a cloud process $\mathcal{N}$ in which the coherences of the input state are not registered and stored, while a completely dephasing download channel has the form $\mathcal{N}=\mathcal{D} \circ \mathcal{N}$ and can be interpreted as a cloud process that fails to output any coherence. Here we consider the worst-case scenario in which both channels channels are completely dephasing. More precisely, we identify a channel $\mathcal{N}_{A}$ with input/output space $A_{0} / A_{1}$ to be free if

$$
\mathcal{N}_{A}=\mathcal{D}_{A_{1}} \circ \mathcal{N}_{A} \circ \mathcal{D}_{A_{0}},
$$


TABLE I. Different classes of free channels in dynamical resource theories of coherence.

\begin{tabular}{lc}
\hline \hline Channel type & \multicolumn{1}{c}{ Definition } \\
\hline Detection Incoherent [27,36] & $\mathcal{D} \circ \mathcal{N}=\mathcal{D} \circ \mathcal{N} \circ \mathcal{D}$ \\
Creation Incoherent (MIO) [21] & $\mathcal{N} \circ \mathcal{D}=\mathcal{D} \circ \mathcal{N} \circ \mathcal{D}$ \\
Detection and Creation & $\mathcal{D} \circ \mathcal{N}=\mathcal{N} \circ \mathcal{D}$ \\
Incoherent (DIO) [23,26] & \\
Incoherent Storage & $\mathcal{N}=\mathcal{N} \circ \mathcal{D}$ \\
Incoherent Output & $\mathcal{N}=\mathcal{D} \circ \mathcal{N}$ \\
Classical [studied here] & $\mathcal{N}=\mathcal{D} \circ \mathcal{N} \circ \mathcal{D}$ \\
\hline \hline
\end{tabular}

where $\mathcal{D}_{A_{0}}$ and $\mathcal{D}_{A_{1}}$ are completely dephasing channels for systems $A_{0}$ and $A_{1}$ in their respective incoherent bases. Note the similarity between the dynamical free objects defined in Eq. (2) and the static free objects in coherence theory. On the level of states, a density operator $\rho$ is incoherent with respect to the fixed basis if

$$
\rho=\mathcal{D}_{A_{1}}(\rho) .
$$

In fact, this can be seen as a special case of Eq. (2) when system $A_{0}$ is one-dimensional.

Channels satisfying Eq. (2) we refer to as classical channels since their action is described entirely by transition probabilities $p(i \mid j)$ from incoherent states $|j\rangle\left\langle\left. j\right|_{A_{0}}\right.$ to incoherent states $|i\rangle\left\langle\left. i\right|_{A_{1}}\right.$. We will denote the set of classical channels that take system $A_{0}$ to $A_{1}$ by $\mathfrak{C}\left(A_{0} \rightarrow A_{1}\right)$,

$$
\mathcal{N}_{A} \in \mathfrak{C}\left(A_{0} \rightarrow A_{1}\right) \Longleftrightarrow \mathcal{N}_{A}=\mathcal{D}_{A_{1}} \circ \mathcal{N}_{A} \circ \mathcal{D}_{A_{0}} .
$$

In particular, the identity channel $\mathrm{id}_{A_{0} \rightarrow A_{1}}$ is not classical as it does not satisfy the above condition (here, $A_{0}$ and $A_{1}$ correspond to the same system in two different temporal or spatial locations and so, $\left.\left|A_{0}\right|=\left|A_{1}\right|\right)$. Physically, the identity channel corresponds to the preservation of coherence for a certain given amount of time. Even though we refer to the our free channels as being "classical," they are still quantum objects. That is, they represent physical processes acting on quantum systems. A summary of different types of channels relevant to different dynamical QRTs of coherence are given in Table I.

Why study a resource theory of classical channels? Here we describe three different motivations. First, some of the most basic nonclassical channels are true resources for quantum information processing. For instance, diagonal unitaries such as the $T$-gate are essential for universal quantum computing. Even the identity channel can be considered as a resource since all physical systems undergo decoherence, and the preservation of coherence in a quantum memory (for some given time or some specified distance [40]) should thus be considered a resource. While both the $T$ gate and the identity are detection incoherent, they are identified as dynamical resources when limiting the free channels to be classical. In this regard, all the nonclassical channels form the dynamical resources of our theory. The quantum Fourier transform (QFT) channel that can generate maximal coherence from free states (due to its unitarity) and preserve entanglement is the maximally coherent channel. (We have shown this analytically in Appendix C by proving that the QFT channel attains the upper bound of log-robustness of coherence of channels which is a monotone in our resource theory.)
Second, quantum cloud computing scenarios like that depicted in Fig. 1 are soon to be physically realized [41]. Having large amounts of noise between the client and cloud computer is to be expected, especially as the spatial separation increases. A highly practical question is then what advantages are possible in the very noisy regime. From this perspective all but the completely dephasing upload/download channels should be deemed as yielding a potential resource for quantum cloud computing. The dynamical QRT we propose here embodies this perspective.

Third, a resource theory in which classical channels constitute the free objects is simple enough that entropic resourcetheoretic measures can be analytically derived. Compared to static resource theories, a plethora of new resource measures arise in dynamical theories, and the abstract theory of these measures has been recently developed [27,29,30,35,37,39,4251]. Unfortunately, the application of this abstract theory to concrete resource theories is usually quite challenging. Here we provide a rare example of a physically motivated resource theory in which, for example, channel-divergence resource measures can actually be computed.

It may be challenged that since the set of classical channels is so small, almost all quantum channels are resources and thus the resource theory considered here offers little physical insight into coherence. However, almost all quantum states are not diagonal in the incoherent basis, and so the same argument could be alleged toward the static resource theory of coherence. Arguably some insight into static quantum coherence has been gained by its recent resource-theoretic development, and so we initiate an effort to attain a similar insight into dynamical quantum coherence. With the free dynamical "states" identified in this resource theory, we now turn to the free operations. This will be some collection of superchannels, which are linear maps that map a quantum channel to another quantum channel even if acting on part of the channel. A superchannel can be realized using a preand a post-processing channel. The details of supermaps and superchannels have been presented in preliminary Sec. II B. For the case of dynamical coherence considered here, the set of free superchannels must map the set of classical channels to itself. Since there are many different superchannels having this property, which ones should be identified as being free? In previous works on dynamical coherence [27,35], the free superchannels were constructed by concatenation of free channels in series or in parallel. In general, this is the most common approach for constructing free superchannels [27,29,30,35,49,52-54]. However, as argued above in the case of static coherence, a free implementation of the allowed operations should not necessarily be required to detect or learn about the resource contained in a state. We now apply this principle on the level of superchannels.

For example, like MIO in the QRT of static coherence, we define as one class of free superchannels the set of maximally incoherent superchannels (MISC), which is the entirety of all superchannnels that do not generate nonclassical channels from classical ones. Similar to MIO in the static case, MISC cannot be implemented without coherence-generating channels. Indeed, if we take the preprocessing channel to be any detection-incoherent channel [as defined in Eq. (1)] and the post-processing channel to be any maximally incoherent 


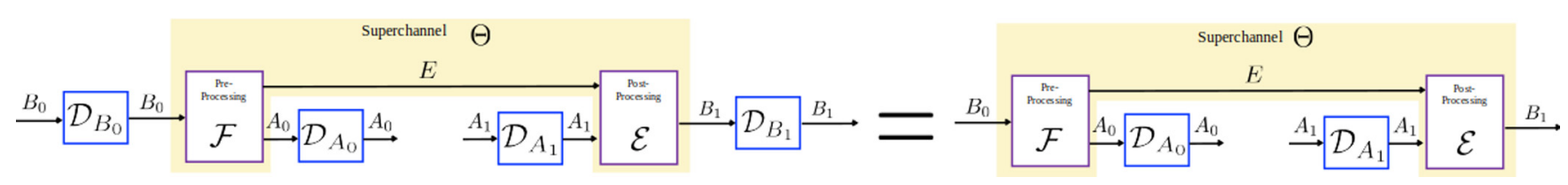

FIG. 2. MISC.

channel, then we obtain a superchannel that belongs to MISC but its pre- and post-processing channels are nonclassical. Nonetheless, much like the argument in static coherence, since we are interested in quantifying the coherence of a channel (as opposed to the coherence of a superchannel), we can use such superchannels as they cannot generate coherence at the channel level, even if it is tensored with the identity superchannel(i.e., it is CRNG).

The bulk of this paper is devoted to developing a resource theory of dynamical coherence based on the ideas just described. This requires borrowing a few mathematical tools, like the concept of log-robustness, the concept of channel divergence, liberal smoothing, etc., from references like Refs. [44,45] that establish the formal structure of dynamical QRTs. Since our work presented here is more mathematical in nature, we would now like to briefly summarize the broad ideas and problems addressed in various sections of this article. This will also serve to highlight the main results of our work.

In Sec. III, we define four different sets of free superchannels: maximally incoherent superchannels (MISC), dephasing-covariant incoherent superchannels (DISC), incoherent superchannels (ISC), and strictly incoherent superchannel (SISC), which are the analog of MIO, DIO, IO, and $\mathrm{SIO}$, in the static case. We focus specifically on the QRTs of MISC and DISC. Similar to how MIO is defined with respect to the dephasing channel, we define MISC with respect to dephasing superchannel, $\Delta$ (whose pre- and post-processing channels are dephasing channels) in the following way:

$\Theta \in \operatorname{MISC}(A \rightarrow B) \Longleftrightarrow \Delta_{B} \circ \Theta_{A \rightarrow B} \circ \Delta_{A}=\Theta_{A \rightarrow B} \circ \Delta_{A}$,

where $\operatorname{MISC}(A \rightarrow B)$ means that the superchannel $\Theta$ converts a quantum channel that takes system $A_{0}$ to $A_{1}$ to another quantum channel that takes system $B_{0}$ to $B_{1}$. Its illustration is given in Fig. 2.

DISC is defined analogously to how DIO is defined in static coherence, i.e.

$$
\Theta \in \operatorname{DISC}(A \rightarrow B) \Longleftrightarrow \Delta_{B} \circ \Theta_{A \rightarrow B}=\Theta_{A \rightarrow B} \circ \Delta_{A},
$$

and its illustration is given in Fig. 3. In our work, we provide simple characterization of MISC and DISC in terms of their Choi matrices in Eqs. (76) and (84), respectively.
In Sec. IV, we study the quantification of dynamical coherence using techniques from QRT of quantum processes $[35,45,53]$ and Sec. $V$ is dedicated to the study the interconversion of channels (i.e., simulation of one channel with another) under MISC and DISC.

In Sec. IV, we first discuss a complete family of monotones under MISC and DISC, and show that these functions can be computed using a semidefinite program. A semidefinite program or an SDP is a subfield of convex optimization. These optimization problems require the variable to be a symmetric matrix which is positive-semidefinite. Section IV B discusses the relative entropies for the quantification of dynamical coherence, which are relevant information quantities to consider for quantum information tasks. Section IV C talks about the montones that have an operational interpretation when we discuss the interconversion of quantum channels. We now list below a few key definitions used in the paper. First, we define the relative entropy of dynamical coherence under MISC to be [for any quantum channel $\mathcal{N}_{A} \in \operatorname{CPTP}\left(A_{0} \rightarrow A_{1}\right)$ ]

$$
\begin{aligned}
C\left(\mathcal{N}_{A}\right):= & \min _{\mathcal{M} \in \mathfrak{C}\left(A_{0} \rightarrow A_{1}\right)} D\left(\mathcal{N}_{A} \| \mathcal{M}_{A}\right) \\
:= & \min _{\mathcal{M} \in \mathfrak{C}\left(A_{0} \rightarrow A_{1}\right)} \max _{\phi \in \mathfrak{D}\left(R_{0} A_{0}\right)} D\left[\mathcal{N}_{A_{0} \rightarrow A_{1}}\left(\phi_{R_{0} A_{0}}\right)\right. \\
& \left.\| \mathcal{M}_{A_{0} \rightarrow A_{1}}\left(\phi_{R_{0} A_{0}}\right)\right],
\end{aligned}
$$

where $\mathfrak{C}\left(A_{0} \rightarrow A_{1}\right)$ denotes the set of all classical channels, $\mathfrak{D}\left(R_{0} A_{0}\right)$ denotes the set of density matrices on system $R_{0} A_{0}$, and $D(\rho \| \sigma)=\operatorname{Tr}[\rho \log \rho-\rho \log \sigma]$ is the quantum relative entropy. This monotone is faithful, i.e., zero iff $\mathcal{N}_{A} \in \mathfrak{C}\left(A_{0} \rightarrow\right.$ $\left.A_{1}\right)$, and does not increase under MISC. For DISC, we define the relative entropy of dynamical coherence to be the function $D_{\Delta}$, given by

$$
D_{\Delta}\left(\mathcal{N}_{A}\right):=D\left(\mathcal{N}_{A} \| \Delta_{A}\left[\mathcal{N}_{A}\right]\right) .
$$

We show that it is a faithful monotone under DISC.

Similarly, the log-robustness of dynamical coherence is defined as

$$
L R_{\mathfrak{C}}\left(\mathcal{N}_{A}\right):=\min _{\mathcal{E} \in \mathfrak{C}\left(A_{0} \rightarrow A_{1}\right)} D_{\max }\left(\mathcal{N}_{A} \| \mathcal{E}_{A}\right)
$$

and the dephasing log-robustness of dynamical coherence as

$$
L R_{\Delta}\left(\mathcal{N}_{A}\right):=D_{\max }\left(\mathcal{N}_{A} \| \Delta_{A}\left[\mathcal{N}_{A}\right]\right) \quad \forall \mathcal{N} \in \operatorname{CPTP}\left(A_{0} \rightarrow A_{1}\right),
$$

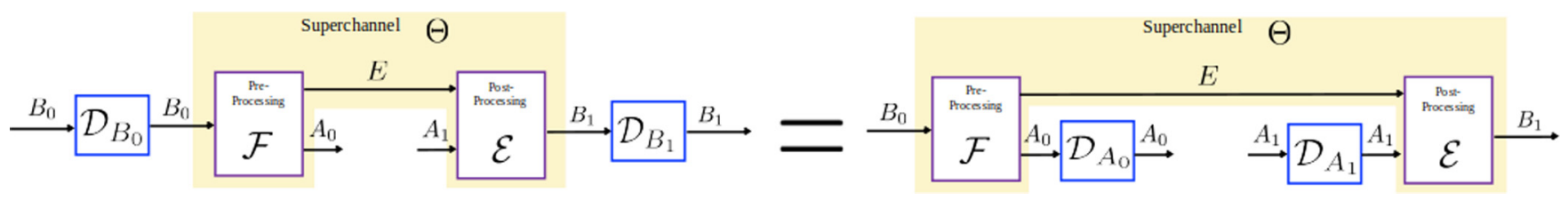

FIG. 3. DISC. 
where $D_{\max }\left(\mathcal{E}_{A} \| \mathcal{F}_{A}\right)$ is the max-relative entropy between two $\mathrm{CP}$ maps $\mathcal{E}_{A}$ and $\mathcal{F}_{A}$ and is discussed in detail in the preliminary Sec. II D. We prove that both these quantities are additive under tensor product and have operational interpretations as the exact dynamical coherence costs in the MISC and DISC cases, respectively. We also compute numerically the logrobustness of coherence for qubit channels and show that in the qubit case, the Hadamard channel attains the maximum value.

In Sec. V, the first subsection, Sec. VA, discusses the general conditions of conversion of one channel using another. This is done by constructing functions called conversion distance such that if the conversion distance from a channel, say $\mathcal{N}_{A}$ to another channel, say $\mathcal{M}_{B}$, is zero, then one can perfectly simulate $\mathcal{M}_{B}$, using $\mathcal{N}_{A}$ and the free superchannels. Moreover, we use a diamond norm to define the interconversion distance, $d_{\mathfrak{F}}\left(\mathcal{N}_{A} \rightarrow \mathcal{M}_{B}\right):=\min _{\Theta \in \mathfrak{F}(A \rightarrow B)} \frac{1}{2}\left\|\Theta_{A \rightarrow B}\left[\mathcal{N}_{A}\right]-\mathcal{M}_{B}\right\|_{\diamond}$ between two quantum channels, $\mathcal{N}_{A} \in \operatorname{CPTP}\left(A_{0} \rightarrow\right.$ $\left.A_{1}\right)$ and $\mathcal{M}_{B} \in \operatorname{CPTP}\left(B_{0} \rightarrow B_{1}\right)$ and show that if the set of free superchannels $\mathfrak{F}=$ MISC or DISC, then $d_{\mathfrak{F}}\left(\mathcal{N}_{A} \rightarrow \mathcal{M}_{B}\right)$ can be computed using a semidefinite program (SDP). The diamond norm [see Eq. (56)] used in the equation of the interconversion distance is used to measure the closeness or the distance between two quantum channels. We use this function to find the interconversion distance in the qubit case, numerically. We find that a maximally coherent replacement channel (a channel that outputs the maximally coherent state $\phi_{B_{1}}^{+}$) can be simulated by a Hadamard channel using the maximally incoherent superchannels. This was expected because any coherent channel can be simulated using the maximally coherent channel and the free superchannels. More interestingly, we also found that we can simulate a Hadamard channel using two maximal replacement channels. Apart from these numerical calculations, we show in Appendix $\mathrm{C}$ that the ratio between the log-robustness of coherence of a maximally coherent channel and the log-robustness of coherence of maximally coherent replacement channel for any dimension is always 2 , i.e., using two maximally replacement channels and the free superchannels, we can simulate the maximally coherent channel which is the quantum Fourier transform channel. Using this fact that we just need two copies of the maximal replacement channel (or two maximally coherent states) to simulate the maximally coherent channel, we can find the coherence cost of a channel using the maximally coherent state. Sections VB, V C, and VD discuss various types of coherence costs of channels. We define the coherence cost of a channel as the minimum amount of the maximally coherent state to be used to simulate the given channel. In Sec. V B, we calculate the exact coherence cost of a channel under MISC and DISC, i.e., when the free superchannel acts on the maximally coherent state the output is the desired channel. Similarly, in Sec. VC, we consider the problem of finding the approximate coherence cost of a channel (which we also refer to as the coherence cost of a channel), i.e., the amount of the maximally coherent state used to convert it to a channel that is very close to the desired channel. This is interesting because experimentally it is extremely difficult to convert one channel into another perfectly. We then, in Sec. VD, compute the liberal asymptotic cost of dynamical coherence (which is the dynamical coherence cost of a channel when the smoothing is "liberal" [45]) under MISC, and show that it is equal to a variant of the regularized relative entropy given by

$$
\begin{aligned}
D_{\mathfrak{C}}^{(\infty)}\left(\mathcal{N}_{A}\right):= & \lim _{n \rightarrow \infty} \frac{1}{n} \sup _{\varphi \in \mathfrak{D}\left(R A_{0}\right)} \min _{\mathcal{E} \in \mathfrak{C}\left(A_{0}^{n} \rightarrow A_{1}^{n}\right)} \\
& \times D\left[\mathcal{N}_{A_{0} \rightarrow A_{1}}^{\otimes n}\left(\varphi_{R A_{0}}^{\otimes n}\right) \| \mathcal{E}_{A_{0}^{n} \rightarrow A_{1}^{n}}\left(\varphi_{R A_{0}}^{\otimes n}\right)\right] .
\end{aligned}
$$

Last, in Sec. VE, we formulate the one-shot distillable dynamical coherence and compute its value for a few specific channels.

\section{PRELIMINARIES}

\section{A. Notations}

In this article, we will denote all the dynamical systems and their corresponding Hilbert spaces by $A, B, C$, etc., and all the static systems and their corresponding Hilbert spaces by $A_{1}, B_{1}, C_{1}$, etc. In this setting, the notation for a dynamical system, say $A$, indicates a pair of systems such that $A=$ $\left(A_{0}, A_{1}\right)=\left(A_{0} \rightarrow A_{1}\right)$ where $A_{0}$ and $A_{1}$ represent the input and output systems, respectively. The choice of notation for the static systems is because all the states can be viewed as channels with trivial input. For a composite system, the notation like $A_{1} B_{1}$ will be used to mean $A_{1} \otimes B_{1}$. To represent the dimension of a system, two vertical lines will be used. For example, the dimension of system $A_{1}$ is $\left|A_{1}\right|$. A replica of the same system would be represented by using a tilde symbol. For instance, system $\tilde{A}_{1}$ is a replica of system $A_{1}$, and system $\tilde{A}_{1} \tilde{B}_{1}$ is a replica of system $A_{1} B_{1}$, i.e., $\left|\tilde{A}_{1}\right|=\left|A_{1}\right|$ and $\left|\tilde{A}_{1} \tilde{B}_{1}\right|=\left|A_{1} B_{1}\right|$.

The set of bounded operators, Hermitian operators, positive operators and density matrices on system $A_{1}$ would be denoted by $\mathfrak{B}\left(A_{1}\right)$, Herm $\left(A_{1}\right), \operatorname{Pos}\left(\mathrm{A}_{1}\right)$, and $\mathfrak{D}\left(A_{1}\right)$, respectively. Note that $\mathfrak{D}\left(A_{1}\right) \subset \operatorname{Pos}\left(\mathrm{A}_{1}\right) \subset \operatorname{Herm}\left(\mathrm{A}_{1}\right) \subset \mathfrak{B}\left(\mathrm{A}_{1}\right)$. Density matrices would be represented by lowercase Greek letters $\rho$, $\sigma$, $\tau$, etc. We will denote the maximally coherent state (or the plus state) by $\phi_{B_{1}}^{+}$for a system $B_{1}$ and the unnormalized maximally entangled states by $\phi_{A_{1} B_{1}}^{+}$for a bipartite system $A_{1} B_{1}$ (note the subscripts in both). The maximally mixed state for a system $B_{1}$ will be denoted by $u_{B_{1}}$. The set of all linear maps from $\mathfrak{B}\left(A_{0}\right)$ to $\mathfrak{B}\left(A_{1}\right)$ would be denoted by $\mathfrak{L}\left(A_{0} \rightarrow A_{1}\right)$, the set of all completely positive maps from $\mathfrak{B}\left(A_{0}\right) \rightarrow \mathfrak{B}\left(A_{1}\right)$ would be denoted by $\mathrm{CP}\left(A_{0} \rightarrow A_{1}\right)$ and the set of quantum channels would be denoted by $\mathrm{CPTP}\left(A_{0} \rightarrow\right.$ $\left.A_{1}\right)$ with $\mathrm{CPTP}\left(A_{0} \rightarrow A_{1}\right) \subset \mathrm{CP}\left(A_{0} \rightarrow A_{1}\right) \subset \mathfrak{L}\left(A_{0} \rightarrow A_{1}\right)$. Throughout this article, we would use calligraphic letters like $\mathcal{E}, \mathcal{F}, \mathcal{M}, \mathcal{N}$, etc., to represent quantum channels. For simplicity, we will denote a quantum channel with a subscript $A$, like $\mathcal{E}_{A}$, to denote an element of $\operatorname{CPTP}\left(A_{0} \rightarrow A_{1}\right)$. The identity map in $\mathfrak{L}\left(A_{0} \rightarrow A_{0}\right)$ will be denoted by $\mathrm{id}_{A_{0}}$.

The notation $\mathfrak{L}(A \rightarrow B)$ will be used to denote the set of all maps from $\mathfrak{L}\left(A_{0} \rightarrow A_{1}\right)$ to $\mathfrak{L}\left(B_{0} \rightarrow B_{1}\right)$. Similarly, the set of all maps from $\operatorname{Herm}\left(A_{0} \rightarrow A_{1}\right)$ to $\operatorname{Herm}\left(B_{0} \rightarrow B_{1}\right)$ would be denoted by $\operatorname{Herm}(A \rightarrow B) \subset \mathfrak{L}(A \rightarrow B)$. All linear maps in $\mathfrak{L}(A \rightarrow B)$ and $\operatorname{Herm}(A \rightarrow B)$ are known as supermaps. We will use capital Greek letters like $\Theta, \Sigma, \Omega$, etc., to denote supermaps. Square brackets will be used to denote the action of supermaps on linear maps. For instance, $\Theta_{A \rightarrow B}\left[\mathcal{E}_{A}\right]$ is 
a linear map in $\mathfrak{L}\left(B_{0} \rightarrow B_{1}\right)$ obtained by the action of a supermap $\Theta \in \mathfrak{L}(A \rightarrow B)$ on a map $\mathcal{E} \in \mathfrak{L}\left(A_{0} \rightarrow A_{1}\right)$. The set of supermaps that map quantum channels to quantum channels (even when tensored with the identity supermap, i.e., even when acting on part of quantum channels) are called superchannels [44,55] and would be represented by $\mathfrak{S}(A \rightarrow$ $B)$. Identity superchannel in $\mathfrak{S}(A \rightarrow A)$ would be denoted by $\mathbb{1}_{A}$. Last, we reserve the symbol $\Delta$ to represent a dephasing superchannel. Such a superchannel converts any channel to a classical channel, and has the dephasing channels as its preand post-processing channels. More detailed description of supermaps and superchannels is provided in the next subsection.

\section{B. Supermaps and superchannels}

The space $\mathfrak{L}\left(A_{0} \rightarrow A_{1}\right)$ is equipped with the following inner product:

$$
\left\langle\mathcal{N}_{A}, \mathcal{M}_{A}\right\rangle:=\sum_{i, j}\left\langle\mathcal { N } _ { A } \left(|i\rangle\left\langle\left. j\right|_{A_{0}}\right), \mathcal{M}_{A}\left(|i\rangle\left\langle\left. j\right|_{A_{0}}\right)\right\rangle_{\mathrm{HS}}\right.\right.
$$

where $\langle X, Y\rangle_{\mathrm{HS}}:=\operatorname{Tr}\left[X^{*} Y\right]$ is the Hilbert-Schmidt inner product between the matrices $X, Y \in \mathfrak{B}\left(A_{1}\right)$. The above inner product is independent of the choice of the orthonormal basis $\{|i\rangle\langle j|\} \in \mathfrak{B}\left(A_{0}\right)$, and can be expressed in terms of Choi matrices. The Choi matrix of a channel $\mathcal{N}_{A}$ is given by

$$
J_{A_{0} A_{1}}^{\mathcal{N}}=\mathcal{N}_{\tilde{A}_{0} \rightarrow A_{1}}\left(\phi_{A_{0} \tilde{A}_{0}}^{+}\right),
$$

where $\phi_{A_{0} \tilde{A}_{0}}^{+} \equiv\left|\phi^{+}\right\rangle\left\langle\left.\phi^{+}\right|_{A_{0} \tilde{A}_{0}}\right.$ is an unnormalized maximally entangled state where $\left|\phi^{+}\right\rangle_{A_{0} \tilde{A}_{0}} \equiv \sum_{i=1}^{\left|A_{0}\right|}|i\rangle_{A_{0} .}|i\rangle_{\tilde{A}_{0}}$. With this notation, the inner product of two channels $\mathcal{N}_{A}$ and $\mathcal{M}_{A}$ can be expressed as the inner product of their Choi matrices, i.e.,

$$
\left\langle\mathcal{N}_{A}, \mathcal{M}_{A}\right\rangle=\left\langle J_{A}^{\mathcal{N}}, J_{A}^{\mathcal{M}}\right\rangle=\operatorname{Tr}\left[\left(J_{A}^{\mathcal{N}}\right)^{*} J_{A}^{\mathcal{M}}\right] .
$$

The canonical orthonormal basis of $\mathfrak{L}(A)$ (relative to the above inner product) is given by $\left\{\mathcal{E}_{A}^{i j k l}\right\}$, where

$$
\mathcal{E}_{A}^{i j k l}\left(\rho_{A_{0}}\right)=\left\langle i\left|\rho_{A_{0}}\right| j\right\rangle|k\rangle\left\langle\left. l\right|_{A_{1}} \quad \forall \rho_{A_{0}} \in \mathfrak{B}\left(A_{0}\right) .\right.
$$

The space $\mathfrak{L}(A \rightarrow B)$ [where $A=\left(A_{0}, A_{1}\right)$ and $B=$ $\left.\left(B_{0}, B_{1}\right)\right]$ is equipped with the following inner product:

$$
\left\langle\Theta_{A \rightarrow B}, \Omega_{A \rightarrow B}\right\rangle:=\sum_{i, j, k, l}\left\langle\Theta_{A \rightarrow B}\left[\mathcal{E}_{A}^{i j k l}\right], \Omega_{A \rightarrow B}\left[\mathcal{E}_{A}^{i j k l}\right]\right\rangle,
$$

where $\Theta_{A \rightarrow B}, \Omega_{A \rightarrow B} \in \mathfrak{L}(A \rightarrow B)$ and the inner product on the right-hand side is the inner product between maps as defined in Eq. (12). The dual of a linear map $\Theta \in \mathfrak{L}(A \rightarrow B)$ is a linear map $\Theta^{*} \in \mathfrak{L}(B \rightarrow A)$ with the property

$$
\left\langle\mathcal{N}_{B}, \Theta\left[\mathcal{M}_{A}\right]\right\rangle=\left\langle\Theta^{*}\left[\mathcal{N}_{B}\right], \mathcal{M}_{A}\right\rangle,
$$

for all $\mathcal{M}_{A} \in \mathfrak{L}(A)$ and for all $\mathcal{N}_{A} \in \mathfrak{L}(B)$.

Similar to how we can express the inner product of two maps by the inner product of their Choi matrices, we can define the inner product of two supermaps as the inner product of their Choi matrices as well. The Choi matrix of a supermap $\Theta_{A \rightarrow B}$ is defined as [44]

$$
\mathbf{J}_{A B}^{\Theta}=\sum_{i, j, k, l} J_{A}^{\mathcal{E}^{i j k l}} \otimes J_{B}^{\Theta\left[\mathcal{E}^{i j k l}\right]},
$$

where $J_{A}^{\mathcal{E}^{i j k l}}$ and $J_{B}^{\Theta\left[\mathcal{E}^{i j k l}\right]}$ are the Choi matrices of $\mathcal{E}_{A}^{i j k l}$ and $\Theta_{A \rightarrow B}\left[\mathcal{E}_{A}^{i j k l}\right]$, respectively. With this notation, the inner product between two supermaps $\Theta_{A \rightarrow B}$ and $\Omega_{A \rightarrow B}$ can be expressed as

$$
\left\langle\Theta_{A \rightarrow B}, \Omega_{A \rightarrow B}\right\rangle=\left\langle\mathbf{J}_{A B}^{\Theta}, \mathbf{J}_{A B}^{\Omega}\right\rangle_{\mathrm{HS}}=\operatorname{Tr}\left[\left(\mathbf{J}_{A B}^{\Theta}\right)^{*} \mathbf{J}_{A B}^{\Omega}\right] .
$$

We now give three alternate expressions of the Choi matrix of the supermap $\Theta \in \mathfrak{L}(A \rightarrow B)$ [44]. First, from its definition, the Choi matrix of a supermap uses the CP map analog of entangled states which we represent as $\mathcal{P}_{A \tilde{A}}^{+}$and is given by

$$
\mathcal{P}_{A \tilde{A}}^{+}=\sum_{i, j, k, l} \mathcal{E}_{A_{0} \rightarrow A_{1}}^{i j k l} \otimes \mathcal{E}_{\tilde{A}_{0} \rightarrow \tilde{A}_{1}}^{i j k l} .
$$

Similar to the properties of the maximally entangled state, the channel $\mathcal{P}_{A \tilde{A}}^{+}$satisfies the following relation for any $\Theta \in$ $\mathfrak{L}(A \rightarrow B)$ :

$$
\Theta_{\tilde{A} \rightarrow B}\left[\mathcal{P}_{A \tilde{A}}^{+}\right]=\Theta_{\tilde{B} \rightarrow A}^{T}\left[\mathcal{P}_{A \tilde{A}}^{+}\right],
$$

where $\Theta^{T} \in \mathfrak{L}(B \rightarrow A)$ is the transpose of the supermap $\Theta$ which is defined by its components,

$$
\begin{aligned}
& \left\langle\mathcal{E}_{A}^{i j k l}, \Theta^{T}\left[\mathcal{E}_{B}^{i^{\prime} j^{\prime} k^{\prime} l^{\prime}}\right]\right\rangle \\
& \quad=\left\langle\mathcal{E}_{B}^{i^{\prime} j^{\prime} k^{\prime} l^{\prime}}, \Theta\left[\mathcal{E}_{A}^{i j k l}\right]\right\rangle \quad \forall i, j, k, l, i^{\prime}, j^{\prime}, k^{\prime}, l^{\prime},
\end{aligned}
$$

where $\left\{\mathcal{E}_{A}^{i j k l}\right\}$ and $\left\{\mathcal{E}_{B}^{i^{\prime} j^{\prime} k^{\prime} l^{\prime}}\right\}$ are the canonical orthonormal basis of $\mathfrak{L}(A)$ and $\mathfrak{L}(B)$, respectively. Then, the Choi matrix of a superchannel $\Theta \in \mathfrak{L}(A \rightarrow B)$ can be expressed as

$$
\mathbf{J}_{A B}^{\Theta}=\Theta\left[\mathcal{P}_{A \tilde{A}}^{+}\right]\left(\phi_{A_{0} \tilde{A}_{0}}^{+} \otimes \phi_{B_{0} \tilde{B}_{0}}^{+}\right) \text {. }
$$

The second way of defining the Choi matrix of a supermap is by noticing its action on the Choi matrices of channels. Lets consider a linear map $\Theta_{A \rightarrow B} \in \mathfrak{L}(A \rightarrow B)$ such that $\mathcal{N}_{B}=$ $\Theta_{A \rightarrow B}\left[\mathcal{M}_{A}\right]$ for some $\mathcal{M}_{A} \in \mathfrak{L}(A)$ and $\mathcal{N}_{B} \in \mathfrak{L}(B)$. Then the Choi matrices of $\mathcal{M}_{A}$ and $\mathcal{N}_{B}$ are related via

$$
J_{B}^{\mathcal{N}}=\operatorname{Tr}_{A}\left[\mathbf{J}_{A B}^{\Theta}\left(\left(J_{A}^{\mathcal{M}}\right)^{T} \otimes I_{B}\right)\right] .
$$

From the above equation, the Choi matrix of the supermap $\Theta$ can be interpreted as the Choi matrix of a linear map $\mathcal{R}_{A \rightarrow B}^{\Theta}$ that takes $J_{A}^{\mathcal{M}}$ to $J_{B}^{\mathcal{N}}$, i.e.

$$
\mathcal{R}_{A \rightarrow B}^{\Theta}\left(J_{A}^{\mathcal{M}}\right)=J_{B}^{\mathcal{N}} .
$$

For the last representation of the Choi matrix of a supermap, we can view it as a linear map $\mathcal{Q}^{\Theta}: \mathfrak{B}\left(A_{1} B_{0}\right) \rightarrow$ $\mathfrak{B}\left(A_{0} B_{1}\right)$ which is defined by the map satisfying

$$
\mathbf{J}_{A B}^{\Theta}:=\mathcal{Q}_{\tilde{A}_{1} \tilde{B}_{0} \rightarrow A_{0} B_{1}}^{\Theta}\left(\phi_{A_{1} \tilde{A}_{1}}^{+} \otimes \phi_{B_{0} \tilde{B}_{0}}^{+}\right) .
$$

We will see that the three representations play a useful role in our study of dynamical resource theory of coherence.

Now let us define a superchannel. A superchannel is a supermap that takes quantum channels to quantum channels even when tensored with identity supermap [44,55-60]. For a linear map $\Theta \in \mathfrak{L}(A \rightarrow B)$, the following are equivalent [44,55]:

(1) $\Theta$ is a superchannel

(2) The Choi matrix $\mathbf{J}_{A B}^{\Theta} \geqslant 0$ with marginals

$$
J_{A_{1} B_{0}}^{\Theta}=I^{A_{1} B_{0}} ; \quad J_{A B_{0}}^{\Theta}=J_{A_{0} B_{0}}^{\Theta} \otimes u_{A_{1}},
$$

where $u_{A_{1}}=\frac{I_{A_{1}}}{\left|A_{1}\right|}$ is the maximally mixed state for system $A_{1}$. 


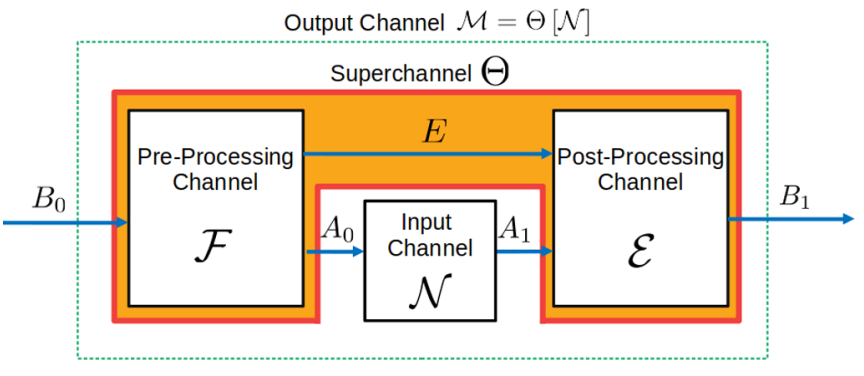

FIG. 4. Realization of a superchannel in terms of pre- and postprocessing channels and its action on an input channel $\mathcal{N}$.

(3) The map $\mathcal{R}_{A \rightarrow B}^{\Theta}$ in Eq. (25) is CP, and there exists a unital CP map $\mathcal{R}_{A_{0} \rightarrow B_{0}}^{\Theta}$ such that the map $\mathcal{R}_{A \rightarrow B_{0}}^{\Theta} \equiv \operatorname{Tr}_{B_{1}} \circ$ $\mathcal{R}_{A \rightarrow B}^{\Theta}$ satisfies

$$
\mathcal{R}_{A \rightarrow B_{0}}^{\Theta}=\mathcal{R}_{A_{0} \rightarrow B_{0}}^{\Theta} \circ \operatorname{Tr}_{A_{1}} .
$$

(4) There exists a Hilbert space $E$, with $|E| \leqslant\left|A_{0} B_{0}\right|$, and two CPTP maps $\mathcal{F} \in \operatorname{CPTP}\left(B_{0} \rightarrow A_{0} E\right)$ and $\mathcal{E} \in$ $\operatorname{CPTP}\left(A_{1} E \rightarrow B_{1}\right)$ such that for all $\mathcal{N}_{A} \in \mathfrak{L}\left(A_{0} \rightarrow A_{1}\right)$,

$$
\Theta\left[\mathcal{N}_{A}\right]=\mathcal{E}_{A_{1} E \rightarrow B_{1}} \circ \mathcal{N}_{A_{0} \rightarrow A_{1}} \circ \mathcal{F}_{B_{0} \rightarrow A_{0} E}
$$

(see Fig. 4).

\section{QRT of static coherence}

Coherence of a state is a basis-dependent concept. Hence, a basis is fixed first in the resource theory of static coherence. The density matrices that are diagonal in this basis form the free states of the theory. These states are also called incoherent states. Let us denote this set by $\mathcal{I}_{A_{1}} \subset \mathfrak{B}\left(A_{1}\right)$ for any system $A_{1}$. Hence, all the incoherent density operators $\rho_{A_{1}} \in \mathcal{I}_{A_{1}}$ have the following form:

$$
\rho_{A_{1}}=\sum_{i=0}^{\left|A_{1}\right|-1} p_{i}|i\rangle\left\langle\left. i\right|_{A_{1}},\right.
$$

with probabilities $p_{i}$ and obey

$$
\mathcal{D}_{A_{1}}\left(\rho_{A_{1}}\right)=\rho_{A_{1}},
$$

where $\mathcal{D}_{A_{1}}$ is the dephasing channel for the system $A_{1}$ and is defined as

$$
\mathcal{D}_{A_{1}}\left(\sigma_{A_{1}}\right)=\sum_{i=0}^{\left|A_{1}\right|-1}|i\rangle\left\langle i\left|\sigma_{A_{1}}\right| i\right\rangle\langle i|
$$

for any $\sigma_{A_{1}} \in \mathfrak{D}\left(A_{1}\right)$. For multipartite systems, the preferred basis is the tensor product of the preferred basis of each subsystem [61-63].

From the golden rule of QRT, the free operations are the set of channels that take the set of incoherent states to itself in the complete sense, i.e., they are completely resource nongenerating. Such operations are called incoherent operations. In literature, several types of incoherent operations have been studied. The largest set of incoherent operations is known as the maximally incoherent operations (MIO) [21]. Other incoherent operations include incoherent operations (IO) [22], dephasing-covariant incoherent operations (DIO)
[23-26], strictly incoherent operations (SIO) [63,64], physically incoherent operations (PIO) [23-25], translationally invariant operations (TIO) [65], genuinely incoherent operations (GIO) [66], fully incoherent operations (FIO) [66], etc. In this section, we will briefly discuss about MIO, DIO, IO, and SIO, as we will be defining four sets of free superchannels in the next section taking their analogy.

The maximally incoherent operations (or MIO) [21] are defined as the set of CPTP and nonselective maps $\mathcal{E} \in \mathfrak{L}\left(A_{0} \rightarrow\right.$ $A_{1}$ ) such that

$$
\mathcal{E}\left(\rho_{A_{0}}\right) \in \mathcal{I}_{A_{1}} \quad \forall \rho_{A_{0}} \in \mathcal{I}_{A_{0}} .
$$

Let us denote the set of all channels that follow the above property by $\mathrm{MIO}\left(\mathrm{A}_{0} \rightarrow \mathrm{A}_{1}\right)$. Any $\mathrm{CPTP}$ map $\mathcal{M}_{A_{0} \rightarrow A_{1}} \in$ $\operatorname{MIO}\left(\mathrm{A}_{0} \rightarrow \mathrm{A}_{1}\right)$ can be characterized using the dephasing channels in the following way

$$
\begin{aligned}
& \mathcal{M}_{A_{0} \rightarrow A_{1}} \\
& \quad \in \operatorname{MIO}\left(\mathrm{A}_{0} \rightarrow \mathrm{A}_{1}\right) \\
& \quad \Longleftrightarrow \mathcal{D}_{A_{1}} \circ \mathcal{M}_{A_{0} \rightarrow A_{1}} \circ \mathcal{D}_{A_{0}}=\mathcal{M}_{A_{0} \rightarrow A_{1}} \circ \mathcal{D}_{A_{0}} .
\end{aligned}
$$

Despite the fact that MIO cannot create coherence, these operations do not have a free dilation, i.e., they cost coherence to be implemented [23-26].

A smaller class of free operations, the incoherent operations (or IO) [22] are defined as the set of CPTP maps $\mathcal{E} \in \operatorname{CPTP}\left(A_{0} \rightarrow A_{1}\right)$ having a Kraus operator representation $\left\{K_{n}\right\}$ such that

$$
\frac{K_{n} \rho_{A_{0}} K_{n}^{\dagger}}{\operatorname{Tr}\left[K_{n} \rho_{A_{0}} K_{n}^{\dagger}\right]} \in \mathcal{I}_{A_{1}} \quad \forall n \text { and } \rho_{A_{0}} \in \mathcal{I}_{A_{0}} .
$$

This class of operations also do not have a free dilation [23-26].

The next class of free operations, the strictly incoherent operations (or SIO) $[63,64]$ are defined as the set of CPTP maps $\mathcal{E} \in \operatorname{CPTP}\left(A_{0} \rightarrow A_{1}\right)$ having a Kraus operator representation $\left\{K_{n}\right\}$ such that

$$
K_{n} \mathcal{D}_{A_{0}}\left(\rho_{A_{0}}\right) K_{n}^{\dagger}=\mathcal{D}_{A_{1}}\left(K_{n} \rho_{A_{0}} K_{n}^{\dagger}\right) \quad \forall n .
$$

This class of operations also do not have a free dilation [23-25].

The last class of free operations that is useful to us is the dephasing-covariant incoherent operations (or DIO) [23-26]. A CPTP map $\mathcal{E}_{A}$ is said to be DIO if

$$
\left[\mathcal{D}, \mathcal{E}_{A}\right]=0
$$

which is equivalent to

$$
\mathcal{D}_{A_{1}}\left[\mathcal{E}_{A_{0} \rightarrow A_{1}}\left(\rho_{A_{0}}\right)\right]=\mathcal{E}_{A_{0} \rightarrow A_{1}}\left[\mathcal{D}_{A_{0}}\left(\rho_{A_{0}}\right)\right] \quad \forall \rho_{A_{0}} \in \mathfrak{D}\left(A_{0}\right) .
$$

\section{Max-relative entropy for channels}

The max-relative entropy is defined on a pair $(\rho, \sigma)$ with $\rho \in \mathfrak{D}\left(A_{1}\right)$ and $\sigma \in \operatorname{Pos}\left(\mathrm{A}_{1}\right)$ of a state $\rho$ with respect to a positive operator $\sigma$ is given by

$$
D_{\max }(\rho \| \sigma):=\log \min \{t: t \sigma \geqslant \rho\},
$$

where the inequality sign means that the difference between the left-hand side (1.h.s.) and right-hand side (r.h.s.) is a 
positive operator. Similarly for channels, the maximum relative entropy between two CP maps $\mathcal{N}$ and $\mathcal{E}$ is given by

$$
D_{\max }\left(\mathcal{N}_{A} \| \mathcal{E}_{A}\right):=\log \min \left\{t: t \mathcal{E}_{A} \geqslant \mathcal{N}_{A}\right\},
$$

where the inequality sign means that the difference between 1.h.s. and r.h.s. is a CP map. Denoting the Choi matrix of $\mathcal{E}_{A}$ by $J_{A}^{\mathcal{E}}$ and that of $\mathcal{N}_{A}$ by $J_{A}^{\mathcal{N}}$, Eq. (40) can be rewritten as

$$
D_{\max }\left(\mathcal{N}_{A} \| \mathcal{E}_{A}\right)=\log \min \left\{t: t J_{A}^{\mathcal{E}} \geqslant J_{A}^{N}, t \geqslant 0\right\} .
$$

The channel max-relative entropy $\left[D_{\max }\left(\mathcal{N}_{A} \| \mathcal{E}_{A}\right)\right]$ can be expressed in a simple closed form as a function of the Choi matrices of the maps $\mathcal{N}_{A}$ and $\mathcal{E}_{A}[48,67]$. This implies that it is also additive under tensor products. For completeness, we give the following proof.

Lemma 1. The max-relative entropy for channels is additive under tensor product, i.e.,

$$
\begin{aligned}
& D_{\max }\left(\mathcal{N}_{A} \otimes \mathcal{M}_{A^{\prime}} \| \mathcal{E}_{A} \otimes \mathcal{F}_{A^{\prime}}\right) \\
& \quad=D_{\max }\left(\mathcal{N}_{A} \| \mathcal{E}_{A}\right)+D_{\max }\left(\mathcal{M}_{A^{\prime}} \| \mathcal{F}_{A^{\prime}}\right) .
\end{aligned}
$$

Proof. For the proof of the inequality $D_{\max }\left(\mathcal{N}_{A} \otimes\right.$ $\left.\mathcal{M}_{A^{\prime}} \| \mathcal{E}_{A} \otimes \mathcal{F}_{A^{\prime}}\right) \leqslant D_{\max }\left(\mathcal{N}_{A} \| \mathcal{E}_{A}\right)+D_{\max }\left(\mathcal{M}_{A^{\prime}} \| \mathcal{F}_{A^{\prime}}\right)$, let

$$
\begin{gathered}
D_{\max }\left(\mathcal{N}_{A} \| \mathcal{E}_{A}\right)=\log \left\{t_{1}: t_{1} \mathcal{E}_{A} \geqslant \mathcal{N}_{A}\right\}, \\
D_{\max }\left(\mathcal{M}_{A^{\prime}} \| \mathcal{F}_{A^{\prime}}\right)=\log \left\{t_{2}: t_{2} \mathcal{F}_{A^{\prime}} \geqslant \mathcal{M}_{A^{\prime}}\right\} .
\end{gathered}
$$

We can rewrite $D_{\max }\left(\mathcal{N}_{A} \otimes \mathcal{M}_{A^{\prime}} \| \mathcal{E}_{A} \otimes \mathcal{F}_{A^{\prime}}\right)$ as

$$
\begin{aligned}
& D_{\max }\left(\mathcal{N}_{A} \otimes \mathcal{M}_{A^{\prime}} \| \mathcal{E}_{A} \otimes \mathcal{F}_{A^{\prime}}\right) \\
& \quad=\log \min \left\{t: t\left(\mathcal{E}_{A} \otimes \mathcal{F}_{A^{\prime}}\right) \geqslant \mathcal{N}_{A} \otimes \mathcal{M}_{A^{\prime}}\right\} \\
& \quad=\log \min \left\{t: \frac{t}{t_{1} t_{2}}\left(t_{1} \mathcal{E}_{A} \otimes t_{2} \mathcal{F}_{A^{\prime}}\right) \geqslant \mathcal{N}_{A} \otimes \mathcal{M}_{A^{\prime}}\right\} .
\end{aligned}
$$

From this, we can clearly see

$$
\log \min \left\{t: t\left(\mathcal{E}_{A} \otimes \mathcal{F}_{A^{\prime}}\right) \geqslant \mathcal{N}_{A} \otimes \mathcal{M}_{A^{\prime}}\right\} \leqslant \log \left(t_{1} t_{2}\right)
$$

Hence,

$$
\begin{aligned}
& D_{\max }\left(\mathcal{N}_{A} \otimes \mathcal{M}_{A^{\prime}} \| \mathcal{E}_{A} \otimes \mathcal{F}_{A^{\prime}}\right) \\
& \quad \leqslant D_{\max }\left(\mathcal{N}_{A} \| \mathcal{E}_{A}\right)+D_{\max }\left(\mathcal{M}_{A^{\prime}} \| \mathcal{F}_{A^{\prime}}\right) .
\end{aligned}
$$

For the proof of $D_{\max }\left(\mathcal{N}_{A} \otimes \mathcal{M}_{A^{\prime}} \| \mathcal{E}_{A} \otimes \mathcal{F}_{A^{\prime}}\right) \geqslant$ $D_{\max }\left(\mathcal{N}_{A} \| \mathcal{E}_{A}\right)+D_{\max }\left(\mathcal{M}_{A^{\prime}} \| \mathcal{F}_{A^{\prime}}\right)$, note that $D_{\max }$ in Eqs. (40) and (41) can be computed using an SDP and its dual is given by

$$
\begin{aligned}
& D_{\max }\left(\mathcal{N}_{A} \| \mathcal{E}_{A}\right) \\
& \quad=\log \max \left\{\operatorname{Tr}\left[\beta_{A} J_{A}^{\mathcal{N}}\right]: \operatorname{Tr}\left[\beta_{A} J_{A}^{\mathcal{E}}\right] \leqslant 1, \beta_{A} \geqslant 0\right\} .
\end{aligned}
$$

Now let $\beta_{A}^{1}$ and $\beta_{A^{\prime}}^{2}$ be optimal for $D_{\max }\left(\mathcal{N}_{A} \| \mathcal{E}_{A}\right)$ and $D_{\max }\left(\mathcal{M}_{A^{\prime}} \| \mathcal{F}_{A^{\prime}}\right)$, respectively. Therefore,

$$
\begin{aligned}
2^{D_{\max }\left(\mathcal{N}_{A} \| \mathcal{E}_{A}\right)} & =\operatorname{Tr}\left[\beta_{A}^{1} J_{A}^{\mathcal{N}}\right], \\
2^{D_{\max }\left(\mathcal{M}_{A^{\prime}} \| \mathcal{F}_{A^{\prime}}\right)} & =\operatorname{Tr}\left[\beta_{A^{\prime}}^{2} J_{A^{\prime}}^{\mathcal{M}}\right] .
\end{aligned}
$$

Using Eq. (48), we can express $2^{D_{\max }\left(\mathcal{N}_{A} \otimes \mathcal{M}_{A^{\prime}} \| \mathcal{E}_{A} \otimes \mathcal{F}_{A^{\prime}}\right)}$ as

$$
2^{D_{\max }\left(\mathcal{N}_{A} \otimes \mathcal{M}_{A^{\prime}} \mid \mathcal{E}_{A} \otimes \mathcal{F}_{A^{\prime}}\right)}=\max \left\{\operatorname{Tr}\left[\beta_{A A^{\prime}}\left(J_{A}^{\mathcal{N}} \otimes J_{A^{\prime}}^{\mathcal{M}}\right)\right]: \operatorname{Tr}\left[\beta_{A A^{\prime}}\left(J_{A}^{\mathcal{E}} \otimes J_{A^{\prime}}^{\mathcal{F}}\right)\right] \leqslant 1, \beta_{A A^{\prime}} \geqslant 1\right\} .
$$

Since the choice of $\beta_{A A^{\prime}}=\beta_{A}^{1} \otimes \beta_{A^{\prime}}^{2}$ satisfies the above constraint, we can say

$$
\begin{aligned}
2^{D_{\max }\left(\mathcal{N}_{A} \otimes \mathcal{M}_{A^{\prime}} \| \mathcal{E}_{A} \otimes \mathcal{F}_{A^{\prime}}\right)} & \geqslant \operatorname{Tr}\left[\beta_{A}^{1} J_{A}^{\mathcal{N}}\right] \operatorname{Tr}\left[\beta_{A^{\prime}}^{2} J_{A^{\prime}}^{\mathcal{M}}\right] \\
& \geqslant 2^{D_{\max }\left(\mathcal{N}_{A} \| \mathcal{E}_{A}\right)} 2^{D_{\max }\left(\mathcal{M}_{A^{\prime}} \| \mathcal{F}_{A^{\prime}}\right)},
\end{aligned}
$$

which implies

$$
\begin{aligned}
& D_{\max }\left(\mathcal{N}_{A} \otimes \mathcal{M}_{A^{\prime}} \| \mathcal{E}_{A} \otimes \mathcal{F}_{A^{\prime}}\right) \\
& \quad \geqslant D_{\max }\left(\mathcal{N}_{A} \| \mathcal{E}_{A}\right)+D_{\max }\left(\mathcal{M}_{A^{\prime}} \| \mathcal{F}_{A^{\prime}}\right) .
\end{aligned}
$$

From Eqs. (47) and (53), we can conclude that the max rel-entropy for channels is additive under tensor products, $\quad$ i.e., $\quad D_{\max }\left(\mathcal{N}_{A} \otimes \mathcal{M}_{A^{\prime}} \| \mathcal{E}_{A} \otimes \mathcal{F}_{A^{\prime}}\right)=$ $D_{\max }\left(\mathcal{N}_{A} \| \mathcal{E}_{A}\right)+D_{\max }\left(\mathcal{M}_{A^{\prime}} \| \mathcal{F}_{A^{\prime}}\right)$.

Last, we would be using $\epsilon$-smooth max-relative entropy in our work which is discussed in detail in $[30,53,68]$ and is defined as (explain the equation below in words) in the following way:

$$
D_{\max }^{\epsilon}\left(\mathcal{N}_{A} \| \mathcal{M}_{A}\right):=\inf _{\mathcal{N}_{A}^{\prime} \in B_{\epsilon}\left(\mathcal{N}_{A}\right)} D_{\max }\left(\mathcal{N}_{A}^{\prime} \| \mathcal{M}_{A}\right),
$$

where

$$
B_{\epsilon}\left(\mathcal{N}_{A}\right)=\left\{\mathcal{N}_{A}^{\prime} \in \operatorname{CPTP}\left(A_{0} \rightarrow A_{1}\right): \frac{1}{2}\left\|\mathcal{N}_{A}^{\prime}-\mathcal{N}_{A}\right\|_{\diamond} \leqslant \epsilon\right\} .
$$

The diamond norm measures the distance between two quantum channels and for any two given channels $\mathcal{E}_{A}$ and $\mathcal{F}_{A}$, it is defined as

$$
\left\|\mathcal{E}_{A}-\mathcal{F}_{A}\right\|_{\diamond}=\max _{\rho}\left\|\mathcal{E}_{\tilde{A_{0}} \rightarrow A_{1}}\left(\rho_{A_{0} \tilde{A_{0}}}\right)-\mathcal{F}_{\tilde{A_{0}} \rightarrow A_{1}}\left(\rho_{A_{0} \tilde{A_{0}}}\right)\right\|_{1},
$$

where $\|\cdot\|_{1}$ represents the trace norm.

\section{THE SET OF FREE SUPERCHANNELS}

As discussed in the introduction, the set of free channels in the theory of dynamical coherence are classical channels. Therefore, a free superchannel consists of a preprocessing classical channel and a post-processing classical channel (see Fig. 5). However, such a free superchannel always destroy completely any resource; that is, it converts all channels (even coherent ones) into classical channels. This means that the resource theory is in a sense "degenerate" and no interesting consequences can be concluded from such a theory.

This above type of degeneracy also occurs with the resource theory of coherence in the state domain. There, the only free operations that are physically consistent are PIO [23], which are very restricted and cannot provide much insight into the phenomenon of coherence in quantum systems. Therefore, almost all the enormous amount of work in recent 


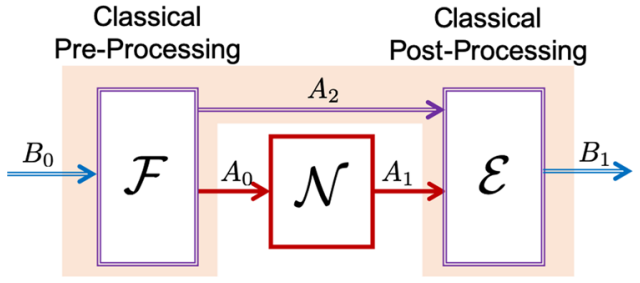

FIG. 5. The action of a classical superchannel on a quantum channel.

years on the QRT of coherence was devoted to the study of coherence under much larger sets of operations, such as MIO, DIO, IO, and SIO. While these larger sets of operations cannot be implemented without a coherence cost, they do not generate coherence, and as such they can be used for the study of coherence of states. However, since MIO, DIO, IO, and SIO, all have a coherence cost, they cannot be used as the "free operations" in a resource theory that aims to quantify the coherence of quantum channels.

Instead, for a dynamical QRT of coherence, one can define free superchannels that form a larger set than classical superchannels. Similar to what happens in the state domain, there is a coherent cost to implement such superchannels; however, they do not generate dynamical coherence, and therefore can be used in a dynamical resource theory of coherence. As it happens in the state domain, there are several natural sets of free superchannels that we can define.

\section{A. Maximally incoherent superchannels (MISC)}

In any quantum resource theory, free operations cannot generate a resource. Taking this principle to the level of superchannels, we define the maximal incoherent superchannels (MISC) as follows.

Definition. Given two dynamical systems $A$ and $B$, a superchannel $\Theta \in \mathfrak{S}(A \rightarrow B)$ is said to be MISC if

$$
\Theta_{A \rightarrow B}\left[\mathcal{N}_{A}\right] \in \mathfrak{C}\left(B_{0} \rightarrow B_{1}\right) \quad \forall \mathcal{N}_{A} \in \mathfrak{C}\left(A_{0} \rightarrow A_{1}\right) .
$$

In other words, a superchannel is said to be MISC if for every input classical channel, the output is also a classical channel. We denote the set of all superchannels that have the above property by $\operatorname{MISC}(A \rightarrow B)$.

Remark. Similar to the characterization of MIO channels with the dephasing channel, the condition that $\Theta$ is in $\operatorname{MISC}(A \rightarrow B)$ can be characterized with the dephasing superchannels $\Delta_{A}$ and $\Delta_{B}$. (A dephasing superchannel can be realized using dephasing channels as pre- and post-processing channels.) Specifically, we have that

$$
\Theta \in \operatorname{MISC}(A \rightarrow B) \Longleftrightarrow \Delta_{B} \circ \Theta_{A \rightarrow B} \circ \Delta_{A}=\Theta_{A \rightarrow B} \circ \Delta_{A} .
$$

That is, for any input quantum channel $\mathcal{E}_{A} \in \operatorname{CPTP}\left(A_{0} \rightarrow\right.$ $A_{1}$ ), if a superchannel $\Theta_{A \rightarrow B}$ obeys the equation on r.h.s. $\left(\Delta_{B} \circ \Theta_{A \rightarrow B} \circ \Delta_{A}\left[\mathcal{E}_{A}\right]=\Theta_{A \rightarrow B} \circ \Delta_{A}\left[\mathcal{E}_{A}\right]\right)$ then, that superchannel belongs to MISC and vice-versa. Refer to Fig. 2 for an illustrative diagram. To explain it further, notice that the dephasing superchannel converts any input to a classical channel. So, for any input quantum channel, say $\mathcal{E}_{A}$, the dephasing superchannel $\Delta_{A}$ first converts it to a classical

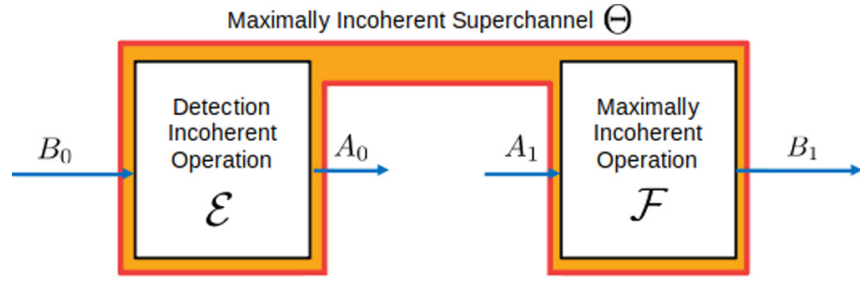

FIG. 6. An example of realization of maximally incoherent superchannel (MISC).

channel, $\Delta_{A}\left[\mathcal{E}_{A}\right]=\mathcal{N}_{A} \in \mathfrak{C}\left(A_{0} \rightarrow A_{1}\right)$ which goes as input to the superchannel $\Theta$. So for a classical channel $\mathcal{N}_{A}$, the r.h.s. of the above condition can be written as $\Delta_{B} \circ \Theta_{A \rightarrow B}\left[\mathcal{N}_{A}\right]=$ $\Theta_{A \rightarrow B}\left[\mathcal{N}_{A}\right]$. The 1.h.s. of this equation again has a dephasing channel which implies that whatever the output is after the action of the superchannel $\Theta$ on the classical channel $\mathcal{N}$, the output would still be classical.

As stated earlier, the maximally incoherent superchannel need not be realized using classical pre- and post-processing channel. For instance, if we use the detection incoherent channels (as defined in Ref. [27]) and maximally incoherent operations (MIO) as the pre- and post-processing channels, then the resultant superchannel is a maximally incoherent superchannel or MISC. Refer to Fig. 6 below as an illustration.

To show that the above realization is really a MISC, we use the fact that an operation $\mathcal{E}_{A} \in \operatorname{CPTP}\left(A_{0} \rightarrow A_{1}\right)$ is called detection incoherent operation iff $\mathcal{D}_{A_{1}} \circ \mathcal{E}_{A}=\mathcal{D}_{A_{1}} \circ \mathcal{E}_{A} \circ \mathcal{D}_{A_{0}}$ [27] where $\mathcal{D}$ is the completely dephasing channel for the given system. Recall that an operation $\mathcal{F}_{A} \in \operatorname{CPTP}\left(A_{0} \rightarrow A_{1}\right)$ is called maximally incoherent operation if it follows Eq. (34). Using these definitions, we can see that the above realization follows Eq. (58) as illustrated in Fig. 7. One of the key properties of any resource theory is that the free operations are "completely free." This is a physical requirement that a free channel (or superchannel) can act on a subsystem. In the following theorem we show that $\operatorname{MISC}(A \rightarrow B)$ is completely free. That is, in the QRT we consider here, there is no difference between RNG and completely RNG.

Theorem 1. Let $A$ and $B$ be two dynamical systems, and let $\Theta \in \operatorname{MISC}(A \rightarrow B)$. Then, for any dynamical system $R$, the superchannel $\mathbb{1}_{R} \otimes \Theta$ is free; i.e. $\mathbb{1}_{R} \otimes \Theta \in \operatorname{MISC}(R A \rightarrow$ $R B)$.

Proof. Let $\mathcal{N}_{R A} \in \mathfrak{C}\left(R_{0} A_{0} \rightarrow R_{1} A_{1}\right)$ be a classical channel satisfying

$$
\Delta_{R A}\left[\mathcal{N}_{R A}\right]=\Delta_{R} \otimes \Delta_{A}\left[\mathcal{N}_{R A}\right]=\mathcal{N}_{R A}
$$

Then,

$$
\begin{aligned}
& \Delta_{R B} \circ\left(\mathbb{1}_{R} \otimes \Theta_{A \rightarrow B}\right)\left[\mathcal{N}_{R A}\right] \\
& \quad=\Delta_{R} \otimes\left(\Delta_{B} \circ \Theta_{A \rightarrow B}\right)\left[\mathcal{N}_{R A}\right] \\
& \quad=\mathbb{1}_{R} \otimes\left(\Delta_{B} \circ \Theta_{A \rightarrow B}\right)\left[\mathcal{N}_{R A}\right] \\
& =\mathbb{1}_{R} \otimes\left(\Delta_{B} \circ \Theta_{A \rightarrow B} \circ \Delta_{A}\right)\left[\mathcal{N}_{R A}\right] \\
& =\mathbb{1}_{R} \otimes\left(\Theta_{A \rightarrow B} \circ \Delta_{A}\right)\left[\mathcal{N}_{R A}\right] \\
& =\mathbb{1}_{R} \otimes \Theta_{A \rightarrow B}\left[\mathcal{N}_{R A}\right],
\end{aligned}
$$

where the first equality follows from the equality $\Delta_{R A}=$ $\Delta_{R} \otimes \Delta_{A}$, the second equality from the fact that $\mathcal{N}_{R A}$ is 


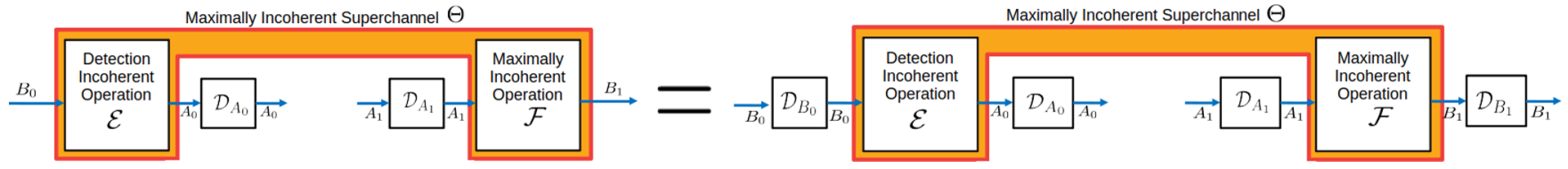

FIG. 7. Illustration to show that this particular realization is a maximally incoherent superchannel (MISC).

classical and in particular $\Delta_{R}\left[\mathcal{N}_{R A}\right]=\mathcal{N}_{R A}$, the third equality from the similar equality $\Delta_{A}\left[\mathcal{N}_{R A}\right]=\mathcal{N}_{R A}$, the fourth equality from Eq. (58), and the last equality follows again from $\Delta_{A}\left[\mathcal{N}_{R A}\right]=\mathcal{N}_{R A}$. Hence, $\mathbb{1}_{R} \otimes \Theta_{A \rightarrow B}\left[\mathcal{N}_{R A}\right]$ is classical so that $\mathbb{1}_{R} \otimes \Theta \in \operatorname{MISC}(R A \rightarrow R B)$. This completes the proof.

The theorem above indicates that MISC can be viewed as the set of completely resource nongenerating superchannels in the theory of dynamical coherence. We next consider the characterization of the set MISC. Recall that in the state domain, we can determine if a channel $\mathcal{E}_{A}$ belongs to $\operatorname{MIO}\left(A_{0} \rightarrow A_{1}\right)$ simply by checking if all the states $\mathcal{E}_{A}\left(|x\rangle\left\langle\left. x\right|_{A_{0}}\right)\right.$ are diagonal for all $x=1, \ldots,\left|A_{0}\right|$. This simplicity of MIO implies that all state conversions in the single-shot regime can be determined with SDP. In the channel domain, however, the characterization of MISC is slightly more complex.

The Choi matrix of any classical channel $\mathcal{N}_{A} \in$ $\operatorname{CPTP}\left(A_{0} \rightarrow A_{1}\right)$ can be expressed as a column stochastic matrix. Recall that the action of this classical channel $\mathcal{N}_{A}$ on any input quantum state $\rho_{A_{0}}$ can be viewed as the action of this column stochastic matrix on a vector whose components are the diagonal entries of the input quantum state $\rho_{A_{0}}$. The output vector's components then form the diagonal entries of the output state and the off-diagonal entries of this output state are zero. The set of all extreme points of the set of $\left|A_{0}\right| \times\left|A_{1}\right|$ column stochastic matrices consists of matrices that in each column has $\left|A_{0}\right|-1$ zeros and 1 one. Therefore, the number of extreme points is given by $\left|A_{0}\right|^{\left|A_{1}\right|}$. This may give the impression that to check if $\Theta \in \operatorname{MISC}\left(A_{0} \rightarrow A_{1}\right)$ one has to check if the channel $\Theta\left[\mathcal{E}_{A}\right]$ is classical for all the $\left|A_{0}\right|^{\left|A_{1}\right|}$ extreme classical channels. Since the number of conditions is exponential in $\left|A_{1}\right|$ it may give the impression that the problem of deciding if a superchannel belongs to MISC cannot be solved with SDP. However, we show now that this problem can be solved with polynomial (in $\left|A_{0} A_{1}\right|$ ) number of constraints. It can be seen from the relationship between the Choi matrix of $\Theta_{A \rightarrow B}$ and that of $\Theta_{A \rightarrow B} \circ \Delta_{A}$ and $\Delta_{B} \circ \Theta_{A \rightarrow B}$.

Lemma 2. Let $A$ and $B$ be two dynamical systems, $\Theta \in$ $\mathfrak{S}(A \rightarrow B)$ be a superchannel, and $\Delta_{A} \in \mathfrak{S}(A \rightarrow A)$ and $\Delta_{B} \in \mathfrak{S}(B \rightarrow B)$ be the completely dephasing superchannels. Then, the Choi matrices of $\Theta_{A \rightarrow B}, \Theta_{A \rightarrow B} \circ \Delta_{A}$, and $\Delta_{B} \circ$ $\Theta_{A \rightarrow B}$, satisfy the relations

$$
\mathbf{J}_{A B}^{\Theta \circ \Delta_{A}}=\mathcal{D}_{A}\left(\mathbf{J}_{A B}^{\Theta}\right) \quad \text { and } \quad \mathbf{J}_{A B}^{\Delta_{B} \odot \Theta}=\mathcal{D}_{B}\left(\mathbf{J}_{A B}^{\Theta}\right) .
$$

Proof. The Choi matrix of a superchannel $\Theta$ can be expressed as the Choi matrix of the bipartite channel $\Theta_{\tilde{A} \rightarrow B}\left[\mathcal{P}^{+}\right]$ [44]. Similarly, the Choi matrix of the superchannel $\Theta \circ \Delta_{A}$ can be expressed as the Choi matrix of the bipartite channel $\Theta_{\tilde{A} \rightarrow B} \circ \Delta_{\tilde{A}}\left[\mathcal{P}_{A \tilde{A}}^{+}\right]$and that of the superchannel $\Delta_{B} \circ \Theta$ as the Choi matrix of $\Delta_{B} \circ \Theta_{\tilde{A} \rightarrow B}\left[\mathcal{P}_{A \tilde{A}}^{+}\right]$.
Denoting $\Theta_{\tilde{A} \rightarrow B}\left[\mathcal{P}_{A \tilde{A}}^{+}\right]$as $\mathcal{N}_{A B}$, the Choi matrix of the superchannel $\Delta_{B} \circ \Theta_{A \rightarrow B}$ can be written as

$$
\begin{aligned}
\mathbf{J}_{A B}^{\Delta_{B} \circ \Theta} & =J_{A B}^{\Delta_{B}\left[\mathcal{N}_{A B}\right]} \\
& =\mathcal{D}_{B_{1}} \circ \mathcal{N}_{\tilde{A}_{0} \tilde{B}_{0} \rightarrow A_{1} B_{1}} \circ \mathcal{D}_{\tilde{B}_{0}}\left(\phi_{A_{0} \tilde{A}_{0}}^{+} \otimes \phi_{B_{0} \tilde{B}_{0}}^{+}\right),
\end{aligned}
$$

where the second equality follows from the definition of the Choi matrix of a channel. Now using the fact that for a given channel $\mathcal{M} \in \operatorname{CPTP}\left(R_{0} \rightarrow R_{1}\right)$, we have $\mathcal{M}_{\tilde{R}_{0} \rightarrow R_{1}}\left|\phi_{R_{0} \tilde{R}_{0}}^{+}\right\rangle=$ $\mathcal{M}_{\tilde{R}_{1} \rightarrow R_{0}}^{T}\left|\phi_{\tilde{R}_{1} R_{1}}^{+}\right\rangle$, we can rewrite Eq. (67) as

$$
\begin{aligned}
\mathbf{J}_{A B}^{\Delta_{B} \circ \Theta} & =\left(\mathcal{D}_{B_{0}} \otimes \mathcal{D}_{B_{1}}\right) \circ \mathcal{N}_{\tilde{A}_{0} \tilde{B}_{0} \rightarrow A_{1} B_{1}}\left(\phi_{A_{0} \tilde{A}_{0}}^{+} \otimes \phi_{B_{0} \tilde{B}_{0}}^{+}\right) \\
& =\mathcal{D}_{B}\left(\mathbf{J}_{A B}^{\Theta}\right) .
\end{aligned}
$$

To find $\mathbf{J}^{\Theta \circ \Delta_{A}}$, note that for any superchannel $\Omega \in \mathfrak{S}(A \rightarrow B)$ we have [44]

$$
\mathbb{1}_{A} \otimes \Omega_{\tilde{A} \rightarrow B}\left[\mathcal{P}_{A \tilde{A}}^{+}\right]=\Omega_{\tilde{B} \rightarrow A}^{T} \otimes \mathbb{1}_{B}\left[\mathcal{P}_{\tilde{B} B}^{+}\right] .
$$

From this, it can be calculated that for the dephasing superchannel, $\Delta^{T}=\Delta$. Therefore, we have

$$
\begin{aligned}
\Theta_{\tilde{A} \rightarrow B} \circ \Delta_{\tilde{A}}\left[\mathcal{P}_{A \tilde{A}}^{+}\right] & =\Theta_{\tilde{A} \rightarrow B} \circ \Delta_{A}^{T}\left[\mathcal{P}_{A \tilde{A}}^{+}\right] \\
& =\Theta_{\tilde{A} \rightarrow B} \circ \Delta_{A}\left[\mathcal{P}_{A \tilde{A}}^{+}\right] \\
& =\Delta_{A} \circ \Theta_{\tilde{A} \rightarrow B}\left[\mathcal{P}_{A \tilde{A}}^{+}\right] \\
& =\Delta_{A} \circ \mathcal{N}_{A B},
\end{aligned}
$$

where the third equality arises because the superchannels $\Theta$ and $\Delta$ are acting on different subsystems (notice the subscripts with and without tilde). So, the Choi matrix of $\Theta_{\tilde{A} \rightarrow B}$ o $\Delta_{\tilde{A}}\left[\mathcal{P}_{A \tilde{A}}^{+}\right]$is equal to finding the Choi matrix of $\Delta_{A} \circ \mathcal{N}_{A B}$. From the calculation of the Choi matrix of $\Delta_{B} \circ \mathcal{N}_{A B}$ above, we can conclude that

$$
\mathbf{J}_{A B}^{\Theta \circ \Delta_{A}}=\mathcal{D}_{A}\left(\mathbf{J}_{A B}^{\Theta}\right) .
$$

With this lemma at hand we get the following characterization for the set $\operatorname{MISC}(A \rightarrow B)$.

Theorem 2. Let $A$ and $B$ be two dynamical systems, and $\Theta \in \mathfrak{S}(A \rightarrow B)$ be a superchannel. Then, $\Theta \in \operatorname{MISC}(A \rightarrow$ $B$ ) if and only if

$$
\mathcal{D}_{A B}\left(\mathbf{J}_{A B}^{\Theta}\right)=\mathcal{D}_{A} \otimes \operatorname{id}_{B}\left(\mathbf{J}_{A B}^{\Theta}\right) .
$$

Proof. The characterization of MISC in Eq. (58) can be equivalently written in terms of the Choi matrices of the channels in 1.h.s. and r.h.s. Using this property and the lemma above we have that

$$
\mathbf{J}_{A B}^{\Delta_{B} \circ \Theta \circ \Delta_{A}}=\mathbf{J}_{A B}^{\Theta^{\circ} \Delta_{A}}=\mathcal{D}_{A} \otimes \operatorname{id}_{B}\left(\mathbf{J}_{A B}^{\Theta}\right) .
$$

Similarly, using both results from Lemma 2, we can write

$$
\mathbf{J}_{A B}^{\Delta_{B} \circ \Theta \circ \Delta_{A}}=\mathrm{id}_{A} \otimes \mathcal{D}_{B}\left(\mathbf{J}_{A B}^{\Theta \circ \Delta_{A}}\right)=\mathcal{D}_{A B}\left(\mathbf{J}_{A B}^{\Theta}\right) .
$$


Equating Eqs. (77) and (78), we get

$$
\mathcal{D}_{A B}\left(\mathbf{J}_{A B}^{\Theta}\right)=\mathcal{D}_{A} \otimes \mathrm{id}_{B}\left(\mathbf{J}_{A B}^{\Theta}\right) .
$$

This completes the proof. have

Note that for any Hermitian matrix $Z_{A B} \in \operatorname{Herm}(A B)$ we

$$
\begin{aligned}
\operatorname{Tr} & {\left[\left(\mathcal{D}_{A B}\left(\mathbf{J}_{A B}^{\Theta}\right)-\mathcal{D}_{A} \otimes \mathrm{id}_{B}\left(\mathbf{J}_{A B}^{\Theta}\right)\right) Z_{A B}\right] } \\
& =\operatorname{Tr}\left[\mathbf{J}_{A B}^{\Theta}\left(\mathcal{D}_{A B}\left(Z_{A B}\right)-\mathcal{D}_{A} \otimes \operatorname{id}_{B}\left(Z_{A B}\right)\right)\right] .
\end{aligned}
$$

Therefore, the theorem above implies that $\Theta \in \operatorname{MISC}(A \rightarrow$ $B$ ) if and only if

$$
\operatorname{Tr}\left[\mathbf{J}_{A B}^{\Theta} X_{A B}\right]=0 \quad \forall X_{A B} \in \mathfrak{K}_{\mathrm{MISC}},
$$

where $\mathfrak{K}_{\text {MISC }}$ is a subspace of $\operatorname{Herm}(A B)$ defined as

$$
\mathfrak{K}_{\mathrm{MISC}}:=\left\{\mathcal{D}_{A B}\left(Z_{A B}\right)-\mathcal{D}_{A} \otimes \operatorname{id}_{B}\left(Z_{A B}\right): Z_{A B} \in \operatorname{Herm}(A B)\right\} .
$$

Since the dimension of the subspace $\mathfrak{K}_{\mathrm{MISC}}$ is $|A B|(|B|-1)$, it is sufficient to restrict $X_{A B}$ in Eq. (81) to the $|A B|(|B|-1)$

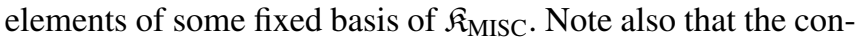
dition above is equivalent to the inclusion $\mathbf{J}_{A B}^{\Theta} \in \mathfrak{K}_{\text {MISC }}^{\perp}$, where $\mathfrak{K}_{\mathrm{MISC}}^{\perp}$ is the orthogonal complement of $\mathfrak{K}_{\text {MISC }}$ in $\operatorname{Herm}(A B)$.

\section{B. Dephasing incoherent superchannels (DISC)}

In the QRT of static coherence, the dephasing channel plays a major role, and in particular, leading to the definition of DIO. Here, the dephasing superchannel defined by $\Delta_{A}\left[\mathcal{N}_{A}\right]=\mathcal{D}_{A_{1}} \circ \mathcal{N}_{A} \circ \mathcal{D}_{A_{0}}$ plays a similar role, as we have already seen in the definition of MISC. We use here the dephasing superchannel to define the set of dephasing incoherent superchannels.

Definition. Let $A$ and $B$ be two dynamical systems, and let $\Theta \in \mathfrak{S}(A \rightarrow B)$ be a superchannel. Then, $\Theta$ is said to be a dephasing incoherent superchannel (DISC) if and only if

$$
\Delta_{B} \circ \Theta_{A \rightarrow B}=\Theta_{A \rightarrow B} \circ \Delta_{A} .
$$

Moreover, the set of all such superchannels that satisfy the above relation is denoted by $\operatorname{DISC}(A \rightarrow B)$.

Clearly, from its definition $\operatorname{DISC}(A \rightarrow B)$ is a subset of $\operatorname{MISC}(A \rightarrow B)$, and in particular, it is completely free. Now, from Lemma 2 it follows that a superchannel $\Theta \in \operatorname{DISC}(A \rightarrow$ $B$ ) if and only if

$$
\mathcal{D}_{A} \otimes \mathrm{id}_{B}\left(\mathbf{J}_{A B}^{\Theta}\right)=\mathrm{id}_{A} \otimes \mathcal{D}_{B}\left(\mathbf{J}_{A B}^{\Theta}\right) .
$$

Moreover, similar to the considerations above, since the map $\mathcal{D}_{A} \otimes \mathrm{id}_{B}-\mathrm{id}_{A} \otimes \mathcal{D}_{B}$ is self adjoint, it follows that $\Theta \in$ $\operatorname{DISC}(A \rightarrow B)$ if and only if

$$
\operatorname{Tr}\left[\mathbf{J}_{A B}^{\Theta} Y_{A B}\right]=0 \quad \forall Y_{A B} \in \mathfrak{K}_{\mathrm{DISC}},
$$

where

$$
\begin{aligned}
\mathfrak{K}_{\mathrm{DISC}}:= & \left\{\operatorname{id}_{A} \otimes \mathcal{D}_{B}\left(Z_{A B}\right)-\mathcal{D}_{A} \otimes \operatorname{id}_{B}\left(Z_{A B}\right)\right. \\
& \left.: Z_{A B} \in \operatorname{Herm}(A B)\right\} .
\end{aligned}
$$

Since the dimension of the subspace $\mathfrak{K}_{\text {DISC }}$ is $|A B|(|A|+|B|-$ 1) it is sufficient to restrict $Y_{A B}$ in Eq. (85) to the $|A B|(|A|+$ $|B|-1)$ elements of some fixed basis of $\mathfrak{K}_{\text {DISC }}$. Note also that the condition above is equivalent to the inclusion $\mathbf{J}_{A B}^{\Theta} \in$ $\mathfrak{K}_{\text {DISC }}^{\perp}$, where $\mathfrak{K}_{\text {DISC }}^{\perp}$ is the orthogonal complement of $\mathfrak{K}_{\text {DISC }}$ in $\operatorname{Herm}(A B)$.

\section{Incoherent superchannels (ISC) and strictly incoherent superchannels (SISC)}

Any superchannel $\Theta \in \mathfrak{S}(A \rightarrow B)$ has a Kraus decomposition, i.e., an operator sum representation,

$$
\Theta_{A \rightarrow B}=\sum_{x=1}^{n} \Theta_{A \rightarrow B}^{x},
$$

where the Choi matrix of each $\Theta_{A \rightarrow B}^{x} \in \mathfrak{L}(A \rightarrow B)$ has rank one. We use this property to define two other sets of free operations that we call incoherent superchannels (ISC) and strictly incoherent superchannels (SISC).

Definition. Let $A$ and $B$ be two dynamical systems, and let $\Theta \in \mathfrak{S}(A \rightarrow B)$ be a superchannel. Then, $\Theta$ is said to be an incoherent superchannel (ISC) if and only if it has a Kraus decomposition $\left\{\Theta_{A \rightarrow B}^{x}\right\}_{x=1}^{n}$ as in Eq. (87) that satisfies

$$
\Delta_{B} \circ \Theta_{A \rightarrow B}^{x} \circ \Delta_{A}=\Theta_{A \rightarrow B}^{x} \circ \Delta_{A} \quad \forall x=1, \ldots, n .
$$

Moreover, the set of all such superchannels that satisfy the above relation is denoted by $\operatorname{ISC}(A \rightarrow B)$.

Definition. Let $A$ and $B$ be two dynamical systems, and let $\Theta \in \mathfrak{S}(A \rightarrow B)$ be a superchannel. Then, $\Theta$ is said to be a strictly incoherent superchannel (SISC) if and only if it has a Kraus decomposition $\left\{\Theta_{A \rightarrow B}^{x}\right\}_{x=1}^{n}$ as in Eq. (87) that satisfies

$$
\Delta_{B} \circ \Theta_{A \rightarrow B}^{x}=\Theta_{A \rightarrow B}^{x} \circ \Delta_{A} \quad \forall x=1, \ldots, n .
$$

Moreover, the set of all such superchannels that satisfy the above relation is denoted by $\operatorname{SISC}(A \rightarrow B)$.

\section{QUANTIFICATION OF DYNAMICAL COHERENCE}

Before we discuss the conversion of one quantum channel into another using free superchannels, it is important to talk quantitatively about the coherence present in quantum channels. This is done by defining functions on quantum channels that map a channel to a real number. A function is called a monotone if it follows the condition of monotonicity which means that the value of the channel should not increase after the channel is acted upon by a free superchannel. To make this function meaningful one more condition is added such that the free channels have the least value. This condition is faithfulness, i.e., the value of such a function should be zero iff the input is a free channel (in our case, the value should be zero for all classical channels). In the problem of interconversion of two resources (using the free superchannels), a resource monotone is specifically useful to tell if one resource can or cannot be converted to another.

Many types of monotones have been defined in literature [15], including general distance-based monotones, entropic monotones, etc. In this section we find the set of monotones to quantify the coherence present in quantum channels. Our work is confined to the case of MISC and DISC. We have divided this section into three subsections. The first subsection gives a complete family of monotones, i.e., to check for the convertibility of one channel to another, it is sufficient to check if the value obtained from all the monotones of this set 
is greater for one channel than other. The second subsection is based on the relative entropies for channels that form a monotone under the free superchannels of our theory. We show that, of the six relative entropies for channels mentioned in [45], only three relative entropies form a monotone in the case of dynamical coherence. The last subsection is dedicated to the monotones that have an operational interpretation in the resource theory of dynamical coherence, i.e., these monotones become meaningful when we find the coherence cost of a channel. One such monotone is known as the log-robustness of coherence of quantum channels. We show that this function can be formulated as an SDP. Using this SDP formulation, we find the upper bound of the log-robustness of coherence of channels in Appendix $\mathrm{C}$ and show that it is achieved by the quantum Fourier transform channel.

\section{A. A complete family of monotones}

In recent works [35,42,44,47,49,51,53,69], various resource measures have been formulated for a general resource theory of channels and for the dynamical resource theory of entanglement. A complete set of monotones for both the general resource theory of channels and the resource theory of entanglement of channels was presented in Ref. [51]. For the conversion of one quantum channel to another using the free superchannels of the theory, it is sufficient to check if all the monotones of this (complete) set acting on one channel are greater than the other. It was shown that the complete family of monotones for the dynamical resource theory of NPT entanglement can be computed using an SDP (which otherwise for LOCC-based entanglement is known to be NP-hard [70]). In general, for a given quantum resource theory, it is not obvious if these functions are computable. In our work, we find a complete set of monotones under the free superchannels MISC and DISC, and show that for the dynamical resource theory of coherence, these functions can be computed using an SDP.

For a general convex quantum resource theory, we can define the following complete set of nonnegative resource measures using any quantum channel $\mathcal{P}_{B} \in \operatorname{CPTP}\left(B_{0} \rightarrow B_{1}\right)$ such that these measures take the value zero on free channels [51],

$$
\begin{aligned}
G_{\mathcal{P}} & \left(\mathcal{N}_{A}\right) \\
:= & \max _{\Theta \in \operatorname{FREE}(A \rightarrow B)}\left\langle\mathcal{P}_{B}, \Theta\left[\mathcal{N}_{A}\right]\right\rangle \\
& -\max _{\mathcal{M}_{B} \in \mathfrak{G}\left(B_{0} \rightarrow B_{1}\right)}\left\langle\mathcal{P}_{B}, \mathcal{M}_{B}\right\rangle \quad \forall \mathcal{N}_{A} \in \operatorname{CPTP}\left(A_{0} \rightarrow A_{1}\right),
\end{aligned}
$$

where $\mathfrak{G}\left(B_{0} \rightarrow B_{1}\right)$ denotes the set of free channels for the given resource theory. Recall that using the free superchannels, one can transform a free channel to any other free channel. Therefore, using some free superchannel $\Theta \in$ $\operatorname{FREE}(A \rightarrow B)$, a given free channel $\mathcal{N}_{A}$ can be converted to the optimal free channel $\mathcal{M}_{B}^{\prime}$ that gives the maximum value for the inner product $\left\langle\mathcal{P}_{B}, \mathcal{M}_{B}^{\prime}\right\rangle$ and, hence, the value of $G_{\mathcal{P}}\left(\mathcal{N}_{A}\right)$ is zero for all free channels.

To construct the complete set of resource monotones for the dynamical resource theory of coherence, we first define a function $f_{\mathcal{P}}\left(\mathcal{M}_{A}\right)$ using a quantum channel $\mathcal{P}_{B} \in$
$\operatorname{CPTP}\left(B_{0} \rightarrow B_{1}\right)$ and superchannel $\Theta \in \mathfrak{F}(A \rightarrow B)$ where $\mathfrak{F}=$ MISC or DISC, as

$$
\begin{aligned}
& f_{\mathcal{P}}\left(\mathcal{M}_{A}\right) \\
& \quad=\max _{\Theta \in \mathfrak{F}(A \rightarrow B)}\left\langle\mathcal{P}_{B}, \Theta\left[\mathcal{M}_{B}\right]\right\rangle \quad \forall \mathcal{M}_{A} \in \operatorname{CPTP}\left(A_{0} \rightarrow A_{1}\right) .
\end{aligned}
$$

Note that Eq. (91) can be expressed as an SDP for a given channel $\mathcal{M}_{A} \in \operatorname{CPTP}\left(A_{0} \rightarrow A_{1}\right)$ in the following manner:

$$
f_{\mathcal{P}}\left(\mathcal{M}_{A}\right)=\max \left\{\operatorname{Tr}\left[\mathbf{J}_{A B}^{\Theta}\left(\left(J_{A}^{\mathcal{M}}\right)^{T} \otimes J_{B}^{\mathcal{P}}\right)\right]\right\},
$$

where the maximum is subject to

$$
\begin{gathered}
\mathbf{J}_{A B}^{\Theta} \geqslant 0, \quad \mathbf{J}_{A B_{0}}^{\Theta}=\mathbf{J}_{A_{0} B_{0}}^{\Theta} \otimes u_{A_{1}}, \quad \mathbf{J}_{A_{1} B_{0}}^{\Theta}=I_{A_{1} B_{0}}, \\
\\
\operatorname{Tr}\left[\mathbf{J}_{A B}^{\Theta} X_{A B}^{i}\right]=0 \forall i=1, \ldots, n .
\end{gathered}
$$

The conditions in Eq. (93) are the conditions on the Choi matrix of the linear map $\Theta$ to be a superchannel. The set of matrices $\left\{X_{A B}^{i}\right\}_{i=1}^{n}$ in Eq. (94) denote the basis of the subspace $\mathfrak{K}_{\mathfrak{F}}$ as defined in Eqs. (82) and (86) for $\mathfrak{F}=$ MISC and DISC, respectively. Accordingly, for MISC, $n \equiv|A B|(|B|-1)$ and for DISC, $n \equiv|A B|(|A|+|B|-1)$.

Similar to Eq. (90) and using any channel $\mathcal{P}_{B} \in$ $\operatorname{CPTP}\left(B_{0} \rightarrow B_{1}\right)$, we can define the complete family of monotones for dynamical coherence in the following way:

$$
G_{\mathcal{P}}\left(\mathcal{N}_{A}\right):=\max \operatorname{Tr}\left[\mathbf{J}_{A B}^{\Theta}\left(\left(J_{A}^{\mathcal{N}}\right)^{T} \otimes J_{B}^{\mathcal{P}}\right)\right]-\max \operatorname{Tr}\left[J_{B}^{\mathcal{M}} J_{B}^{\mathcal{P}}\right]
$$

where we have expressed the inner product between channels as the inner product of the Choi matrices of the respective channels. The first maximum in Eq. (95) is subject to the constraints given in Eqs. (93) and (94) and the second maximum is over all classical channels $\mathcal{M}_{B} \in \mathfrak{C}\left(B_{0} \rightarrow B_{1}\right)$. The family $\left\{G_{\mathcal{P}}\right\}$ over all $\mathcal{P} \in \operatorname{CPTP}\left(B_{0} \rightarrow B_{1}\right)$ form a complete set of monotones, that is, there exists a $\Theta \in \mathfrak{F}(A \rightarrow B)$ where $\mathfrak{F}=$ MISC or DISC, that can convert a channel $\mathcal{E}_{A} \in \operatorname{CPTP}\left(A_{0} \rightarrow\right.$ $\left.A_{1}\right)$ to another channel $\mathcal{F}_{B} \in \operatorname{CPTP}\left(B_{0} \rightarrow B_{1}\right)$ if and only if

$$
G_{\mathcal{P}}\left(\mathcal{E}_{A}\right) \geqslant G_{\mathcal{P}}\left(\mathcal{F}_{B}\right)
$$

Remark 1. For the qubit case we calculated the values of the monotone $G_{\mathcal{P}}\left(\mathcal{N}_{A}\right)$ under MISC for a few channels (or a class of channels) by plugging into CVX. This required construction of 48 basis elements [Eq. (82)]. The value of $G_{\mathcal{P}}\left(\mathcal{N}_{A}\right)$ for all classical channels is 0 for all $\mathcal{P}_{B}$. We found that for a fixed $\mathcal{P}_{B}$, the value of all unitary channels is the same and they attain the maximum value of 2 when $\mathcal{P}_{B}$ is the identity channel. If we fix $\mathcal{P}_{B}$ to be the identity channel, then we see that for a replacement channel that outputs a plus state $\left(|+\rangle=\frac{1}{\sqrt{n}} \sum_{i=0}^{n-1}|i\rangle\right)$, the value of $G_{\text {id }}\left(\mathcal{N}_{A}\right)$ is equal to 2 . For any other replacement channel and any depolarizing channel, $G_{\text {id }}\left(\mathcal{N}_{A}\right)$ is less than 2 .

Remark 2. Since there are an infinite number of monotones in the above complete set $G_{\mathcal{P}}$, it might give an impression that the conversion of a channel $\mathcal{N}_{A} \in \operatorname{CPTP}\left(A_{0} \rightarrow A_{1}\right)$ to another channel $\mathcal{M}_{B} \in \operatorname{CPTP}\left(B_{0} \rightarrow B_{1}\right)$ using a superchannel $\Theta \in$ MISC or DISC, is very hard or impractical, but in Sec. V we show that the problem of interconversion of two quantum 
channels using a superchannel belonging to MISC or DISC can be computed using an SDP.

\section{B. Relative entropies of dynamical coherence}

A measure of distinguishability or divergence $D(\cdot \| \cdot)$ of two states is a function $D: \mathfrak{D}\left(A_{1}\right) \times \mathfrak{D}\left(B_{1}\right) \rightarrow \mathbb{R}$ such that it obeys data-processing inequality and is zero on the set of free states. One example of such a function is Rényi divergence [71]. Its two generalizations which have been given an operational interpretation are "Sandwiched" Rényi relative entropy (also known as quantum Rényi divergence) and Petz-Rényi relative entropy. "Sandwiched" Rényi relative entropy (or quantum Rényi divergence) was introduced and discussed in Refs. [72-75]. It is based on a parameter $\alpha \in$ $[0, \infty]$ and reduces to the relative von Neumann entropy for $\alpha=1$, to the relative max entropy for $\alpha=\infty$, and closely related to the fidelity $\operatorname{Tr}\left(\sigma^{1 / 2} \rho \sigma^{1 / 2}\right)^{1 / 2}$ for $\alpha=1 / 2$, where $\rho$ and $\sigma$ are two density matrices which are input to the entropy function. Also, the "Sandwiched" Rényi relative entropy is a monotone under quantum operations, i.e., satisfies the dataprocessing inequality for $\alpha \in[1 / 2, \infty]$ [75]. The Petz-Rényi relative entropy was introduced and studied in Refs. [76-78] and it finds an operational interpretation in the context of quantum hypothesis testing. Therefore, these two are relevant information quantities to consider for quantum information theory. Other generalizations of the Rényi divergence and the quantum Rényi relative entropies are discussed in Ref. [79] but their operational meaning is not clear.

For the channel case (i.e., dynamical resources), the relative entropies and divergence have been generalized from the state case (i.e., static resources) and were discussed in Refs. [35,44,45,48,53,80,81]. The channel divergence for two given channels $\mathcal{N}_{A}, \mathcal{M}_{A} \in \operatorname{CPTP}\left(A_{0} \rightarrow A_{1}\right)$ is defined as Refs. [44,80,81],

$D\left(\mathcal{N}_{A} \| \mathcal{M}_{A}\right)=\max _{\phi \in \mathfrak{D}\left(R_{0} A_{0}\right)} D\left[\mathcal{N}_{A_{0} \rightarrow A_{1}}\left(\phi_{R_{0} A_{0}}\right) \| \mathcal{M}_{A_{0} \rightarrow A_{1}}\left(\phi_{R_{0} A_{0}}\right)\right]$,

where $D(\rho \| \sigma)=\operatorname{Tr}[\rho \log \rho-\rho \log \sigma]$ is the relative entropy. We take the relative entropies listed in [45] and find the following three relative entropies to be clearly forming a monotone under MISC

$$
\begin{aligned}
C_{1}\left(\mathcal{N}_{A}\right)= & \min _{\mathcal{M} \in \mathfrak{C}\left(A_{0} \rightarrow A_{1}\right)} \max _{\phi \in \mathfrak{D}\left(R_{0} A_{0}\right)} \\
& \times D\left[\mathcal{N}_{A_{0} \rightarrow A_{1}}\left(\phi_{R_{0} A_{0}}\right) \| \mathcal{M}_{A_{0} \rightarrow A_{1}}\left(\phi_{R_{0} A_{0}}\right)\right], \\
C_{2}\left(\mathcal{N}_{A}\right)= & \min _{\mathcal{M} \in \mathfrak{C}\left(A_{0} \rightarrow A_{1}\right)} \sup _{\rho, \sigma \in \mathfrak{D}\left(R_{0} A_{0}\right)} D\left[\mathcal{N}_{A}\left(\rho_{R_{0} A_{0}}\right) \| \mathcal{M}_{A}\left(\sigma_{R_{0} A_{0}}\right)\right] \\
& -D\left(\rho_{R_{0} A_{0}} \| \sigma_{R_{0} A_{0}}\right), \\
C_{3}\left(\mathcal{N}_{A}\right)= & \max _{\rho \in \mathfrak{D}\left(R_{0} A_{0}\right)} D\left[\mathcal{N}_{A}\left(\rho_{R_{0} A_{0}}\right)\right]-D\left(\rho_{R_{0} A_{0}}\right),
\end{aligned}
$$

where $D(\rho)=\min _{\mathcal{D}(\sigma)=\sigma} D(\rho \| \sigma)$. The proof that the above relative entropies form a monotone under MISC is similar to the proof for relative entropies forming a monotone for a general resource theory of quantum processes as given in Ref. [45] and are quite straightforward. For completeness, we give the proof of monotonicity for one of the above monotones $C_{1}$ and the proof for the other two follows likewise. Let $\Theta_{A \rightarrow B} \in \operatorname{MISC}(A \rightarrow B)$. Then the relative entropy of the channel $\Theta\left[\mathcal{N}_{A}\right] \in \operatorname{CPTP}\left(B_{0} \rightarrow B_{1}\right)$ for some channel $\mathcal{N}_{A} \in$ $\operatorname{CPTP}(A \rightarrow B)$ can be written as

$$
\begin{aligned}
C_{1}\left(\Theta\left[\mathcal{N}_{A}\right]\right) & =\min _{\mathcal{M}} \max _{\mathcal{C}\left(B_{0} \rightarrow B_{1}\right)} D\left[\max _{\phi \in \mathfrak{D}\left(R_{0} B_{0}\right)} D\left[\Theta_{A \rightarrow B}\left[\mathcal{N}_{A}\right]\left(\phi_{R_{0} B_{0}}\right) \| \mathcal{M}_{B_{0} \rightarrow B_{1}}^{\prime}\left(\phi_{R_{0} B_{0}}\right)\right]\right. \\
& \leqslant \min _{\mathcal{M} \in \mathfrak{C}\left(A_{0} \rightarrow A_{1}\right)} \max _{\phi \in \mathfrak{D}\left(R_{0} B_{0}\right)} D\left[\Theta_{A \rightarrow B}\left[\mathcal{N}_{A}\right]\left(\phi_{R_{0} B_{0}}\right) \| \Theta_{A \rightarrow B}\left[\mathcal{M}_{A}\right]\left(\phi_{R_{0} B_{0}}\right)\right] \\
& \leqslant \min _{\mathcal{M} \in \mathfrak{C}\left(A_{0} \rightarrow A_{1}\right)} \max _{\rho \in \mathfrak{D}\left(R_{0} A_{0}\right)} D\left[\mathcal{N}_{A}\left(\rho_{R_{0} A_{0}}\right) \| \mathcal{M}_{A}\left(\rho_{R_{0} A_{0}}\right)\right] \\
& =C_{1}\left(\mathcal{N}_{A}\right),
\end{aligned}
$$

where the first inequality follows because the relative entropy of the channel $\Theta\left[\mathcal{N}_{A}\right]$ is the minimum taken over all classical channels in system $B$ and the second inequality follows because of the data-processing inequality. Note that the relative entropies $C_{1}\left(\mathcal{N}_{A}\right)$ and $C_{2}\left(\mathcal{N}_{A}\right)$ are faithful, i.e., they take the value zero iff $\mathcal{N}_{A} \in \mathfrak{C}\left(A_{0} \rightarrow A_{1}\right)$. The relative entropy $C_{3}\left(\mathcal{N}_{A}\right)$ is a state-based relative entropy and involves no optimization over the classical channels.

In Ref. [45], there are three other relative entropies that are defined similar to $C_{1}, C_{2}$, and $C_{3}$, where the optimization is taken over the set of free states instead of all density matrices. There, the proof relies on the preprocessing channel to be completely resource nongenerating. Since, we cannot make this assumption (because in the dynamical resource theory of quantum coherence, such a channel would completely destroy any resource), hence, we cannot say anything about the mono- tonicity of the relative entropies where the optimization is over the incoherent states.

To define the resource monotones for DISC, we first define the function $D_{\Delta}:$ CPTP $\rightarrow \mathbb{R}_{+}$as follows for any channel divergence $D$ :

$$
D_{\Delta}\left(\mathcal{N}_{A}\right):=D\left(\mathcal{N}_{A} \| \Delta_{A}\left[\mathcal{N}_{A}\right]\right),
$$

and for the choice $D=D_{\max }$ we call it the dephasing logrobustness and denote it by $D_{\Delta} \equiv L R_{\Delta}$.

Lemma 3. The function $D_{\Delta}$ is a dynamical resource monotones under DISC.

Proof. Lets $\Theta \in \operatorname{DISC}(A \rightarrow B)$ and $\mathcal{N} \in \operatorname{CPTP}\left(A_{0} \rightarrow\right.$ $\left.A_{1}\right)$. Then,

$$
\begin{aligned}
D_{\Delta}\left(\Theta_{A \rightarrow B}\left[\mathcal{N}_{A}\right]\right) & =D\left(\Theta_{A \rightarrow B}\left[\mathcal{N}_{A}\right] \| \Delta_{B} \circ \Theta_{A \rightarrow B}\left[\mathcal{N}_{A}\right]\right) \\
& =D\left(\Theta_{A \rightarrow B}\left[\mathcal{N}_{A}\right] \| \Theta_{A \rightarrow B} \circ \Delta_{A}\left[\mathcal{N}_{A}\right]\right)
\end{aligned}
$$




$$
\begin{aligned}
& \leqslant D\left(\mathcal{N}_{A} \| \Delta_{A}\left[\mathcal{N}_{A}\right]\right) \\
& =D_{\Delta}\left(\mathcal{N}_{A}\right) .
\end{aligned}
$$

This completes the proof.

For the case that $D(\rho \| \sigma)=\operatorname{Tr}[\rho \log \rho]-\operatorname{Tr}[\rho \log \sigma]$ is the relative entropy, we call $D_{\Delta}$ the dephasing relative entropy of coherence.

\section{Operational monotones}

This subsection is dedicated to the discussion of the operationally relevant monotones for the resource theory of dynamical coherence. The monotones discussed here are operationally meaningful in a sense that these monotones are useful for calculating various costs of coherent channels. Hence, this subsection can be treated as a preliminary to the next section where we talk about the interconversion of two coherent channels. We will see that the monotones which are based on $D_{\max }$, like various types of log-robustness, play a major role in the calculation of coherence cost of channels.

The log-robustness of entanglement for states was introduced and investigated in [82-85]. It was shown that it is an entanglement monotone and its operational significance for the manipulation of entanglement was also discussed. The log-robustness of coherence for states was similarly defined in [86] and it was shown that it is a measure of coherence. For a state $\rho_{A_{1}} \in \mathfrak{D}\left(A_{1}\right)$, it is defined as follows:

$$
L R_{\mathcal{I}}\left(\rho_{A_{1}}\right):=\min _{\sigma_{A_{1}} \in \mathcal{I}_{A_{1}}} D_{\max }\left(\rho_{A_{1}} \| \sigma_{A_{1}}\right),
$$

where $\mathcal{I}_{A_{1}}$ is the set of incoherent states for system $A_{1}$. The log-robustness of channels for a general resource theory was introduced and discussed in Refs. [35,45,53]. It was shown that the log-robustness of channels satisfy necessary conditions for the resource measure of channels, i.e., it is both faithful (means, it gives the value zero for free channels, and is greater than zero otherwise) and a monotone under tensoring, and left and right compositions with free channels [53]. Likewise, we can define the log-robustness of coherence of channels, which is a monotone under MISC, in the following way:

$$
L R_{\mathfrak{C}}\left(\mathcal{N}_{A}\right):=\min _{\mathcal{E} \in \mathfrak{C}\left(A_{0} \rightarrow A_{1}\right)} D_{\max }\left(\mathcal{N}_{A} \| \mathcal{E}_{A}\right),
$$

where the minimum is taken over all the classical channels. The proof that it is a monotone is very similar to the proof presented for the relative entropy $C_{1}$ [see Eq. (101)] and follows easily using the data-processing inequality. Besides, it can be computed with an SDP. To see why, note that

$$
\begin{aligned}
L R_{\mathfrak{C}}\left(\mathcal{N}_{A}\right)= & \log \min \left\{t \geqslant 0: t \mathcal{E}_{A} \geqslant \mathcal{N}_{A},\right. \\
& \left.\Delta_{A}\left[\mathcal{E}_{A}\right]=\mathcal{E}_{A}, \quad \mathcal{E} \in \operatorname{CPTP}\left(A_{0} \rightarrow A_{1}\right)\right\},
\end{aligned}
$$

where the first condition arises from the definition of $D_{\max }$ for channels, and the second and third arise from the requirement of $\mathcal{E}$ to be a classical channel. Denoting by $\omega_{A}$ the Choi matrix of $t \mathcal{E}_{A}$ we get that (recall that we are using $u$ to denote the maximally mixed state)

$$
\begin{gathered}
L R_{\mathfrak{C}}\left(\mathcal{N}_{A}\right)=\log \min \left\{\frac{1}{\left|A_{0}\right|} \operatorname{Tr}\left[\omega_{A}\right]: \omega_{A} \geqslant J_{A}^{\mathcal{N}}, \mathcal{D}_{A}\left[\omega_{A}\right]=\omega_{A},\right. \\
\left.\omega_{A_{0}}=\operatorname{Tr}\left[\omega_{A}\right] u_{A_{0}}, \omega_{A} \geqslant 0\right\},
\end{gathered}
$$

which is an SDP optimization problem. As such it has a dual given by (see the Appendix for details)

$$
\begin{aligned}
L R_{\mathfrak{C}}\left(\mathcal{N}_{A}\right)= & \log \max \left\{\operatorname{Tr}\left[\eta_{A} J_{A}^{\mathcal{N}}\right]: \mathcal{D}_{A}\left(\eta_{A}\right)=\mathcal{D}_{A_{0}}\left(\eta_{A_{0}}\right) \otimes u_{A_{1}},\right. \\
& \left.\mathcal{D}_{A_{1}}\left[\eta_{A_{1}}\right]=I_{A_{1}}, \quad \eta_{A} \geqslant 0\right\} .
\end{aligned}
$$

Remark. For the qubit case, we calculated the logrobustness of coherence of few channels. For any classical channel, the log-robustness of coherence is equal to 0 . For the identity channel it is equal to 1 . For any replacement channel and depolarizing channel, its value is between 0 and 1 . If the replacement channel is the one that outputs the plus state $\left(|+\rangle=\frac{1}{\sqrt{n}} \sum_{i=0}^{n-1}|i\rangle\right)$, then the log-robustness is equal to 1 . For any unitary channel, we found that the value of log-robustness of coherence is between 1 and 2 . We found that the value obtained for the Hadamard gate is the maximum and is equal to 2 . In these examples, we can see that a quantum channel can have the ability to preserve and/or generate coherence. For instance, replacement channels cannot preserve coherence but can only generate coherence whereas the identity channel can only preserve coherence but not generate coherence. The unitary channels can do both, i.e., they can preserve coherence as well as they can generate coherence. This fact explains the higher value of the log-robustness of coherence of the unitary channels as compared to the replacement channels. Also, as calculated in Appendix $\mathrm{C}$, the quantum Fourier transform channel has the maximum value for this monotone. This can be explained from the fact that a QFT channel can generate the maximally coherent state from a free state and has the ability to preserve entanglement. (Note that the replacement channels are entanglement-breaking channels.)

Next, we show the additivity of log-robustness of coherence of channels under tensor products. This result is useful when we go to the asymptotic limit.

Lemma 4. The log-robustness of coherence of a channel is additive under tensor products, i.e.,

$$
L R_{\mathfrak{C}}\left(\mathcal{N}_{A} \otimes \mathcal{M}_{A^{\prime}}\right)=L R_{\mathfrak{C}}\left(\mathcal{N}_{A}\right)+L R_{\mathfrak{C}}\left(\mathcal{M}_{A^{\prime}}\right) .
$$

Proof. For the proof of the inequality $L R_{\mathfrak{C}}\left(\mathcal{N}_{A} \otimes \mathcal{M}_{A^{\prime}}\right) \leqslant$ $L R_{\mathfrak{C}}\left(\mathcal{N}_{A}\right)+L R_{\mathfrak{C}}\left(\mathcal{M}_{A^{\prime}}\right)$, let $L R_{\mathfrak{C}}\left(\mathcal{N}_{A}\right)=D_{\max }\left(\mathcal{N}_{A} \| \mathcal{E}_{A}\right)$ and $L R_{\mathfrak{C}}\left(\mathcal{M}_{A^{\prime}}\right)=D_{\max }\left(\mathcal{M}_{A^{\prime}} \| \mathcal{E}_{A^{\prime}}\right)$ for some optimal $\mathcal{E}_{A}$ and $\mathcal{E}_{A^{\prime}}$. Then, we have

$$
\begin{aligned}
L R_{\mathfrak{C}}\left(\mathcal{N}_{A} \otimes \mathcal{M}_{A^{\prime}}\right) & \leqslant D_{\max }\left(\mathcal{N}_{A} \otimes \mathcal{M}_{A^{\prime}} \| \mathcal{E}_{A} \otimes \mathcal{E}_{A^{\prime}}\right) \\
& =D_{\max }\left(\mathcal{N}_{A} \| \mathcal{E}_{A}\right)+D_{\max }\left(\mathcal{M}_{A^{\prime}} \| \mathcal{E}_{A^{\prime}}\right) \\
& =L R_{\mathfrak{C}}\left(\mathcal{N}_{A}\right)+L R_{\mathfrak{C}}\left(\mathcal{M}_{A^{\prime}}\right)
\end{aligned}
$$

The first inequality follows trivially from the definition of logrobustness and the second equality follows from the additivity of $D_{\max }$.

To prove the converse, i.e., $L R_{\mathfrak{C}}\left(\mathcal{N}_{A} \otimes \mathcal{M}_{A^{\prime}}\right) \geqslant$ $L R_{\mathfrak{C}}\left(\mathcal{N}_{A}\right)+L R_{\mathfrak{C}}\left(\mathcal{M}_{A^{\prime}}\right)$, we will use the dual of the log-robustness as given in Eq. (108). Let $\eta_{A}$ and $\eta_{A^{\prime}}$ be the optimal matrices for the dual of $L R_{\mathfrak{C}}\left(\mathcal{N}_{A}\right)$ and $L R_{\mathfrak{C}}\left(\mathcal{M}_{A^{\prime}}\right)$, respectively. Then we have

$$
2^{L R_{\mathfrak{C}}\left(\mathcal{N}_{A}\right)}=\operatorname{Tr}\left[\eta_{A} J_{A}^{\mathcal{N}_{A}}\right], \quad 2^{L R_{\mathfrak{C}}\left(\mathcal{M}_{A^{\prime}}\right)}=\operatorname{Tr}\left[\eta_{A^{\prime}} J_{A^{\prime}}^{\mathcal{M}_{A^{\prime}}}\right] .
$$


Since, $L R_{\mathfrak{C}^{(}}\left(\mathcal{N}_{A} \otimes \mathcal{M}_{A^{\prime}}\right)=\log \max \operatorname{Tr}\left[\eta_{A A^{\prime}}^{\prime}\left(J_{A A^{\prime}}^{\mathcal{N}_{A} \otimes \mathcal{M}_{A^{\prime}}}\right)\right]$ where the maximum is over all $\eta_{A A^{\prime}}^{\prime} \geqslant 0$, satisfying

$$
\mathcal{D}_{A A^{\prime}}\left(\eta_{A A^{\prime}}^{\prime}\right)=\mathcal{D}_{A_{0} A_{0}^{\prime}}\left(\eta_{A_{0} A_{0}^{\prime}}^{\prime}\right) \otimes u_{A_{1} A_{1}^{\prime}}, \quad \mathcal{D}_{A_{1} A_{1}^{\prime}}\left[\eta_{A_{1} A_{1}^{\prime}}^{\prime}\right]=I_{A_{1} A_{1}^{\prime}} .
$$

For the particular choice of $\eta_{A A^{\prime}}=\eta_{A} \otimes \eta_{A^{\prime}}$, it is easy to verify that it satisfies the above conditions and therefore for this choice of $\eta_{A A^{\prime}}$ we have

$$
2^{L R_{\mathfrak{C}}\left(\mathcal{N}_{A} \otimes \mathcal{M}_{A^{\prime}}\right)} \geqslant \operatorname{Tr}\left[\eta_{A A^{\prime}}\left(J_{A A^{\prime}}^{\mathcal{N}_{A} \otimes \mathcal{M}_{A^{\prime}}}\right)\right]=2^{L R_{\mathfrak{C}}\left(\mathcal{N}_{A}\right)} 2^{L R_{\mathfrak{C}}\left(\mathcal{M}_{A^{\prime}}\right)} .
$$

In the above equation, the first inequality follows from the dual of the log-robustness of channels and the second equality is because $\eta_{A}$ and $\eta_{A^{\prime}}$ are optimal for $L R_{\mathfrak{C}}\left(\mathcal{N}_{A}\right)$ and $L R_{\mathfrak{C}}\left(\mathcal{M}_{A^{\prime}}\right)$. Hence, the above equation implies

$$
L R_{\mathfrak{C}}\left(\mathcal{N}_{A} \otimes \mathcal{M}_{A^{\prime}}\right) \geqslant L R_{\mathfrak{C}}\left(\mathcal{N}_{A}\right)+L R_{\mathfrak{C}}\left(\mathcal{M}_{A^{\prime}}\right)
$$

This establishes the additivity of the log-robustness of a quantum channel, i.e., $L R_{\mathfrak{C}}\left(\mathcal{N}_{A} \otimes \mathcal{M}_{A^{\prime}}\right)=L R_{\mathfrak{C}}\left(\mathcal{N}_{A}\right)+$ $L R_{\mathfrak{C}}\left(\mathcal{M}_{A^{\prime}}\right)$.

Another type of log-robustness, the dephasing logrobustness, which will be used to find the exact cost under DISC, is defined by

$$
L R_{\Delta}\left(\mathcal{N}_{A}\right):=D_{\max }\left(\mathcal{N}_{A} \| \Delta_{A}\left[\mathcal{N}_{A}\right]\right) \quad \forall \mathcal{N} \in \operatorname{CPTP}\left(A_{0} \rightarrow A_{1}\right)
$$

The dephasing log-robustness is a monotone under DISC which is easy to show using Eq. (83) and data-processing inequality. While the log-robustness behaves monotonically under any superchannel in MISC, the dephasing log-robustness is in general not monotonic under MISC. Instead, it is monotonic under DISC.

Lemma 5. For any $\mathcal{N} \in \operatorname{CPTP}\left(A_{0} \rightarrow A_{1}\right)$ and $\Theta \in$ $\operatorname{DISC}(A \rightarrow B)$ we have

$$
L R_{\Delta}\left(\Theta_{A \rightarrow B}\left[\mathcal{N}_{A}\right]\right) \leqslant L R_{\Delta}\left(\mathcal{N}_{A}\right) .
$$

Proof.

$$
\begin{aligned}
L_{\Delta}\left(\Theta_{A \rightarrow B}\left[\mathcal{N}_{A}\right]\right) & =D_{\max }\left(\Theta_{A \rightarrow B}\left[\mathcal{N}_{A}\right] \| \Delta_{A} \circ \Theta_{A \rightarrow B}\left[\mathcal{N}_{A}\right]\right) \\
& =D_{\max }\left(\Theta_{A \rightarrow B}\left[\mathcal{N}_{A}\right] \| \Theta_{A \rightarrow B} \circ \Delta_{A}\left[\mathcal{N}_{A}\right]\right) \\
& \leqslant D_{\max }\left(\mathcal{N}_{A} \| \Delta_{A}\left[\mathcal{N}_{A}\right]\right) \\
& =L R_{\Delta}\left(\mathcal{N}_{A}\right),
\end{aligned}
$$

where the second equality follows from the commutativity of $\Theta$ and $\Delta$, and the inequality follows from the data processing inequality of the channel divergence $D_{\max }$ [44].

We prove here that the dephasing log-robustness is also additive.

Lemma 6. Let $\mathcal{N} \in \operatorname{CPTP}\left(A_{0} \rightarrow A_{1}\right) \quad$ and $\quad \mathcal{M} \in$ $\operatorname{CPTP}\left(B_{0} \rightarrow B_{1}\right)$ be two channels. Then,

$$
L R_{\Delta}\left(\mathcal{N}_{A} \otimes \mathcal{M}_{B}\right)=L R_{\Delta}\left(\mathcal{N}_{A}\right)+L R_{\Delta}\left(\mathcal{M}_{B}\right)
$$

Proof.

$$
\begin{aligned}
& L R_{\Delta}\left(\mathcal{N}_{A} \otimes \mathcal{M}_{B}\right) \\
& \quad=D_{\max }\left(\mathcal{N}_{A} \otimes \mathcal{M}_{B} \| \Delta_{A B}\left[\mathcal{N}_{A} \otimes \mathcal{M}_{B}\right]\right) \\
& \quad=D_{\max }\left(\mathcal{N}_{A} \otimes \mathcal{M}_{B} \| \Delta_{A}\left[\mathcal{N}_{A}\right] \otimes \Delta_{B}\left[\mathcal{M}_{B}\right]\right)
\end{aligned}
$$

$$
\begin{aligned}
= & D_{\max }\left(\mathcal{N}_{A} \| \Delta_{A}\left[\mathcal{N}_{A}\right]\right) \\
& +D_{\max }\left(\mathcal{M}_{B} \| \Delta_{B}\left[\mathcal{M}_{B}\right]\right) \\
= & L R_{\Delta}\left(\mathcal{N}_{A}\right)+L R_{\Delta}\left(\mathcal{M}_{B}\right),
\end{aligned}
$$

where the third equality follows from the additivity of $D_{\max }$ for channels.

We also define smoothed log-robustness and asymptotic log-robustness which would be useful in finding the approximate and liberal coherence costs of a channel whose meanings are discussed in detail in the next section. From Ref. [45], we know that smoothing maintains monotonicity. Thus, the smoothed log-robustness which is defined below is also a monotone

$$
L R_{\mathfrak{C}}^{\epsilon}\left(\mathcal{N}_{A}\right):=\min _{\mathcal{N}^{\prime} \in B_{\epsilon}\left(\mathcal{N}_{A}\right)} L R_{\mathfrak{C}}\left(\mathcal{N}_{A}^{\prime}\right),
$$

where the minima is taken over the log-robustness of all channels that lie within the $\epsilon$-ball given by the diamond norm. This $\epsilon$-ball, $B_{\epsilon}\left(\mathcal{N}_{A}\right)$, around the channel $\mathcal{N}_{A}$ is defined as

$$
B_{\epsilon}\left(\mathcal{N}_{A}\right)=\left\{\mathcal{N}^{\prime} \in \operatorname{CPTP}\left(A_{0} \rightarrow A_{1}\right): \frac{1}{2}\left\|\mathcal{N}_{A}^{\prime}-\mathcal{N}_{A}\right\|_{\diamond} \leqslant \epsilon\right\} .
$$

To obtain the asymptotic log-robustness, we first regularize the smoothed log-robustness and then take the limit $\epsilon \rightarrow 0^{+}$. Thus, the asymptotic log-robustness is defined as

$$
L R_{\mathfrak{C}}^{\infty}\left(\mathcal{N}_{A}\right)=\lim _{\epsilon \rightarrow 0^{+}} \liminf _{n \rightarrow \infty} \frac{1}{n} L R_{\mathfrak{C}^{(}}^{\epsilon}\left(\mathcal{N}_{A}^{\otimes n}\right) .
$$

Similarly we define the smoothed dephasing logrobustness and asymptotic dephasing log-robustness, both of which are monotones under DISC. The smoothed dephasing log-robustness is defined by

$$
L R_{\Delta}^{\epsilon}\left(\mathcal{N}_{A}\right):=\min _{\mathcal{N}^{\prime} \in B_{\epsilon}\left(\mathcal{N}_{A}\right)} L R_{\Delta}\left(\mathcal{N}_{A}^{\prime}\right)
$$

and the asymptotic dephasing log-robustness as

$$
L R_{\Delta}^{\infty}\left(\mathcal{N}_{A}\right)=\lim _{\epsilon \rightarrow 0^{+}} \lim _{n \rightarrow \infty} \frac{1}{n} L R_{\Delta}^{\epsilon}\left(\mathcal{N}_{A}^{\otimes n}\right) .
$$

Now we define the log-robustness with "liberal" smoothing [45] which we find to have an operational meaning. Let

$$
L R_{\mathfrak{C}}^{\epsilon, \varphi}\left(\mathcal{N}_{A}\right):=\min _{\mathcal{N}^{\prime} \in B_{\epsilon}^{\varphi}\left(\mathcal{N}_{A}\right)} L R_{\mathfrak{C}}\left(\mathcal{N}_{A}^{\prime}\right)
$$

where

$$
\begin{aligned}
B_{\epsilon}^{\varphi}\left(\mathcal{N}_{A}\right):= & \left\{\mathcal{N}^{\prime} \in \operatorname{CP}\left(A_{0} \rightarrow A_{1}\right):\right. \\
& \left.\left\|\mathcal{N}_{A}^{\prime}\left(\varphi_{R A_{0}}\right)-\mathcal{N}_{A}\left(\varphi_{R A_{0}}\right)\right\|_{1} \leqslant \epsilon\right\},
\end{aligned}
$$

and consider its "liberal" smoothing

$$
L R_{\mathfrak{C}^{\mathfrak{C}}}^{\epsilon}\left(\mathcal{N}_{A}\right):=\max _{\varphi \in \mathcal{D}\left(R A_{0}\right)} L R_{\mathfrak{C}}^{\epsilon, \varphi}\left(\mathcal{N}_{A}\right) .
$$

Define also

$$
L R_{\mathfrak{C}}^{\epsilon, n}\left(\mathcal{N}_{A}\right):=\frac{1}{n} \max _{\varphi \in \mathcal{D}\left(R A_{0}\right)} L R_{\mathfrak{C}}^{\epsilon, \varphi^{\otimes n}}\left(\mathcal{N}_{A}^{\otimes n}\right)
$$

and

$$
L R_{\mathfrak{C}}^{(\infty)}\left(\mathcal{N}_{A}\right):=\lim _{\epsilon \rightarrow 0^{+}} \liminf _{n \rightarrow \infty} L R_{\mathfrak{C}}^{\epsilon, n}\left(\mathcal{N}_{A}\right)
$$


In Ref. [45], a new type of regularized relative entropy of a resource given by

$$
\begin{aligned}
D_{\mathfrak{C}}^{(\infty)}\left(\mathcal{N}_{A}\right):= & \lim _{n \rightarrow \infty} \frac{1}{n} \sup _{\varphi \in \mathfrak{D}\left(R A_{0}\right)} \min _{\mathcal{E} \in \mathfrak{C}\left(A_{0}^{n} \rightarrow A_{1}^{n}\right)} \\
& \times D\left[\mathcal{N}_{A_{0} \rightarrow A_{1}}^{\otimes n}\left(\varphi_{R A_{0}}^{\otimes n}\right) \| \mathcal{E}_{A_{0}^{n} \rightarrow A_{1}^{n}}\left(\varphi_{R A_{0}}^{\otimes n}\right)\right] .
\end{aligned}
$$

The quantity $D_{\mathfrak{C}}^{(\infty)}\left(\mathcal{N}_{A}\right)$ behaves monotonically under completely RNG superchannels and satisfies the following asymptotic equipartition property (AEP),

$$
L R_{\mathfrak{C}}^{(\infty)}\left(\mathcal{N}_{A}\right)=D_{\mathfrak{C}}^{(\infty)}\left(\mathcal{N}_{A}\right) .
$$

\section{INTERCONVERSIONS}

As the name suggests, in this section we discuss the conversion of one channel into another. Conversion of one resource to another using the set of free operations is one of the major tasks of resource theory [15]. This conversion can be of various types like single shot (exact or approximate) interconversion, asymptotic interconversion, catalytic interconversion, etc. Addressing this leads to two very interesting questions. The first is the problem of finding the cost, i.e., minimum amount of maximal resource necessary to output a given resource using free operations, and the second is distillation which is the inverse problem of cost, i.e., asking how much maximal resource can be extracted from a given resource using the free operations. We answer these questions in this section for the resource theory of dynamical coherence. Note that since we just require two copies of the maximally coherent replacement channel to simulate the maximally coherent channel (refer to Appendix C), we use the maximally coherent replacement channel to compute the cost and distillation.

This section is broadly divided into three parts namely, the conversion distance of coherence, cost of a channel, and the problem of distilling an arbitrary channel into pure-state coherence. The first part is discussed in Sec. V A where we take up the task of conversion of one channel into another using MISC or DISC. For this task, we form a function called the conversion distance, and claim that if the conversion distance is very small, then, we can simulate one channel using the other with the help of free superchannels. We show that for the dynamical resource theory of coherence, the conversion distance $d_{\mathfrak{F}}\left(\mathcal{N}_{A} \rightarrow \mathcal{M}_{B}\right)$ for two given channels $\mathcal{N}_{A}$ and $\mathcal{M}_{B}$ can be computed with an SDP. We then take up the problem of finding various costs of coherence which are discussed in Secs. V B, V C, and VD. In these three subsections, we calculate the exact, approximate and "liberal" coherence cost of a channel and show that the "liberal" cost of coherence is equal to a variant of the regularized relative entropy. In the last Sec. VE, we calculate one-shot distillable rates and end the subsection by providing examples of the distillable rates for partial depolarizing channel and partial dephasing channel.

\section{A. The conversion distance of coherence}

The conversion distance from a channel $\mathcal{N}_{A} \in$ $\operatorname{CPTP}\left(A_{0} \rightarrow A_{1}\right)$ to a channel $\mathcal{M}_{B} \in \operatorname{CPTP}\left(B_{0} \rightarrow B_{1}\right)$ is defined as (with $\mathfrak{F}$ standing for either one of the four operations MISC, DISC, ISC, and SISC)

$$
d_{\mathfrak{F}}\left(\mathcal{N}_{A} \rightarrow \mathcal{M}_{B}\right):=\min _{\Theta \in \mathfrak{F}(A \rightarrow B)} \frac{1}{2}\left\|\Theta_{A \rightarrow B}\left[\mathcal{N}_{A}\right]-\mathcal{M}_{B}\right\|_{\diamond} .
$$

Recall that the diamond norm is used to measure the distance of two quantum channels and is defined in Eq. (56). Therefore, if the conversion distance from a channel $\mathcal{N}_{A}$ to another channel $\mathcal{M}_{B}$ is very small, then $\Theta_{A \rightarrow B}\left[\mathcal{N}_{A}\right]$ is very close to $\mathcal{M}_{B}$, which implies that $\mathcal{N}_{A}$ can be used to simulate $\mathcal{M}_{B}$ using free superchannels. We now show that for $\mathfrak{F}=$ MISC or $\mathfrak{F}=$ DISC, this conversion distance can be computed with a semidefinite program (SDP).

Theorem 3. Let $\left\{X_{A B}^{i}\right\}_{i=1}^{n}$ be the basis of the subspace $\mathfrak{K}_{\mathfrak{F}}$ as defined in (82) where $n \equiv|A B|(|B|-1)$ for the case $\mathfrak{F}=$ MISC and $n \equiv|A B|(|A|+|B|-1)$ for the case $\mathfrak{F}=$ DISC. Let $\alpha_{A B}$ denote the Choi matrix of the superchannel $\Theta$. Then, $d_{\mathfrak{F}}\left(\mathcal{N}_{A} \rightarrow \mathcal{M}_{B}\right)$, can be expressed as the following SDP:

$$
d_{\mathfrak{F}}\left(\mathcal{N}_{A} \rightarrow \mathcal{M}_{B}\right)=\min \lambda,
$$

where the minimum is subject to

$$
\begin{gathered}
\lambda I_{B_{0}} \geqslant \omega_{B_{0}}, \quad \omega_{B} \geqslant 0, \quad \alpha_{A B} \geqslant 0, \\
\omega_{B} \geqslant \operatorname{Tr}_{A}\left[\alpha_{A B}\left(\left(J_{A}^{\mathcal{N}}\right)^{T} \otimes I_{B}\right)\right]-J_{B}^{\mathcal{M}}, \\
\alpha_{A B_{0}}=\alpha_{A_{0} B_{0}} \otimes u_{A_{1}}, \alpha_{A_{1} B_{0}}=I_{A_{1} B_{0}}, \\
\operatorname{Tr}\left[\alpha_{A B} X_{A B}^{i}\right]=0 \forall i=1, \ldots, n .
\end{gathered}
$$

Proof. The proof of the above theorem depends on the fact that the diamond norm can be expressed as an SDP [87] and is presented in detail in Appendix B.

Remark 1. The code to calculate the interconversion distance from one channel to another is given in Ref. [88].

Remark 2. For MISC, the interconversion distance from the Hadamard channel to the maximal replacement channel (i.e., the channel that outputs a maximally coherent state $\phi_{B_{1}}^{+}$ for any input) is 0 , i.e., it is possible to construct a protocol to convert the Hadamard channel to maximal replacement channel using the maximally incoherent superchannel. We also found that the interconversion distance from two maximal replacement channels to a Hadamard channel is also 0 which was expected from the results of Appendix C.

\section{B. Exact asymptotic coherence cost}

The exact single-shot coherence cost of a channel $\mathcal{N}_{A} \in$ $\operatorname{CPTP}\left(A_{0} \rightarrow A_{1}\right)$ is defined as the log of the minimum dimension of the maximally coherent state which can be used to convert to the given channel using a free superchannel and can be expressed as

$$
\begin{aligned}
C_{\mathfrak{F}}^{0}\left(\mathcal{N}_{A}\right):= & \min \left\{\log \left|R_{1}\right|: \exists \Theta \in \mathfrak{F}\left(R_{1} \rightarrow A\right) \quad\right. \text { s.t. } \\
& \left.\Theta_{R_{1} \rightarrow A}\left[\phi_{R_{1}}^{+}\right]=\mathcal{N}_{A}\right\},
\end{aligned}
$$

where the zero on the superscript of $C$ on 1.h.s. means that the conversion is exact. In our work, we consider the two cases of $\mathfrak{F}=$ MISC and $\mathfrak{F}=$ DISC. The exact asymptotic coherence cost is defined by regularizing the exact single-shot cost which 
is given as

$$
C_{\mathfrak{F}}^{\text {exact }}\left(\mathcal{N}_{A}\right)=\lim _{n \rightarrow \infty} \frac{1}{n} C_{\mathfrak{F}}^{0}\left(\mathcal{N}_{A}^{\otimes n}\right) .
$$

We now compute this exact coherence cost for both MISC and DISC.

\section{Exact cost under MISC}

Theorem 4. The exact coherence cost of a channel $\mathcal{N}_{A} \in$ $\operatorname{CPTP}\left(A_{0} \rightarrow A_{1}\right)$ under $\mathfrak{F}=$ MISC is given by

$$
C_{\mathfrak{F}}^{\text {exact }}\left(\mathcal{N}_{A}\right)=L R_{\mathfrak{C}}\left(\mathcal{N}_{A}\right) .
$$

Proof. To prove the above theorem we first prove that

$$
L R_{\mathfrak{C}}\left(\mathcal{N}_{A}\right) \leqslant C_{\mathfrak{F}}^{0}\left(\mathcal{N}_{A}\right) \leqslant L R_{\mathfrak{C}}\left(\mathcal{N}_{A}\right)+1,
$$

and then we regularize the above condition and use the additivity of $L R_{\mathfrak{C}}\left(\mathcal{N}_{A}\right)$ to find the exact asymptotic cost of a channel.

For the proof of $L R_{\mathfrak{C}}(\mathcal{N}) \leqslant C_{\mathfrak{F}}^{0}(\mathcal{N})$, let $\Theta \in \operatorname{MISC}\left(R_{1} \rightarrow\right.$ $A)$ be an optimal superchannel satisfying $\Theta_{R_{1} \rightarrow A}\left[\phi_{R_{1}}^{+}\right]=\mathcal{N}_{A}$ such that $C_{\text {MISC }}^{0}\left(\mathcal{N}_{A}\right)=\log _{2}\left|R_{1}\right|$. Therefore,

$$
\begin{aligned}
L R_{\mathfrak{C}}\left(\mathcal{N}_{A}\right) & =D_{\max }\left(\mathcal{N}_{A} \| \mathcal{E}_{A}\right) \\
& =D_{\max }\left(\Theta_{R_{1} \rightarrow A}\left[\phi_{R_{1}}^{+}\right] \| \mathcal{E}_{A}\right) \\
& \leqslant D_{\max }\left(\Theta_{R_{1} \rightarrow A}\left[\phi_{R_{1}}^{+}\right] \| \Theta_{R_{1} \rightarrow A}\left[\mathcal{D}\left(\phi_{R_{1}}^{+}\right)\right]\right) \\
& \leqslant D_{\max }\left(\phi_{R_{1}}^{+} \| \mathcal{D}\left(\phi_{R_{1}}^{+}\right)\right) \\
& =\log _{2}\left|R_{1}\right| \\
& =C_{\mathfrak{F}}^{0}\left(\mathcal{N}_{A}\right),
\end{aligned}
$$

where the first equality follows from the definition of logrobustness of a channel [see Eq. (105)] for some optimal classical channel $\mathcal{E}_{A}$. The first inequality above arises because we have chosen the optimal $\mathcal{E}_{A}$ such that $D_{\max }\left(\mathcal{N}_{A} \| \mathcal{E}_{A}\right)$ is minimum. The second inequality, i.e., Eq. (146) follows from the data-processing inequality. The equality in Eq. (147) can be be easily computed following Eq. (39).

To prove $C_{\mathfrak{F}}^{0}\left(\mathcal{N}_{A}\right) \leqslant L R_{\mathfrak{C}}\left(\mathcal{N}_{A}\right)+1$, first let

$$
L R_{\mathfrak{C}}\left(\mathcal{N}_{A}\right)=D_{\max }\left(\mathcal{N}_{A} \| \mathcal{E}_{A}\right)=\log _{2} t
$$

for some optimal $t$ satisfying $t \mathcal{E}_{A} \geqslant \mathcal{N}_{A}$. Also, let $m=\lceil t\rceil$, so that $m \mathcal{E}_{A} \geqslant \mathcal{N}_{A}$ still holds. Let $R_{1}$ be a static system such that $\left|R_{1}\right|=m$. We now define the following supermap. For any state $\rho_{R_{1}} \in \mathfrak{D}\left(R_{1}\right)$,

$$
\begin{aligned}
\Omega_{R_{1} \rightarrow A}\left[\rho_{R_{1}}\right]:= & \frac{m}{m-1}\left(\operatorname{Tr}\left[\phi_{R_{1}}^{+} \rho_{R_{1}}\right]-\frac{1}{m}\right) \mathcal{N}_{A} \\
& +\frac{m}{m-1}\left(1-\operatorname{Tr}\left[\phi_{R_{1}}^{+} \rho_{R_{1}}\right]\right) \mathcal{E}_{A} .
\end{aligned}
$$

Note that the supermap $\Omega_{R_{1} \rightarrow A} \in \mathfrak{F}\left(R_{1} \rightarrow A\right)$ as it can be expressed as

$$
\begin{aligned}
\Omega_{R_{1} \rightarrow A}\left[\rho_{R_{1}}\right]:= & \operatorname{Tr}\left[\phi_{R_{1}}^{+} \rho_{R_{1}}\right] \mathcal{N}_{A} \\
& +\frac{1}{m-1}\left(1-\operatorname{Tr}\left[\phi_{R_{1}}^{+} \rho_{R_{1}}\right]\right)\left(m \mathcal{E}_{A}-\mathcal{N}_{A}\right),
\end{aligned}
$$

where $m \mathcal{E}_{A}-\mathcal{N}_{A} \geqslant 0$. Also observe that $\Omega_{R_{1} \rightarrow A}\left(\phi_{R_{1}}^{+}\right)=\mathcal{N}_{A}$. Hence, such a superchannel implies that

$$
C_{\mathfrak{F}}^{0}\left(\mathcal{N}_{A}\right)=\log _{2} m=\log _{2}\lceil t\rceil \leqslant \log _{2} t+1=L R_{\mathfrak{C}}\left(\mathcal{N}_{A}\right)+1 .
$$

This completes the proof of $L R_{\mathfrak{C}}\left(\mathcal{N}_{A}\right) \leqslant C_{\mathfrak{F}}^{0}\left(\mathcal{N}_{A}\right) \leqslant$ $L R_{\mathfrak{C}}\left(\mathcal{N}_{A}\right)+1$.

Therefore, using regularization and the additivity of $L R_{\mathfrak{C}}\left(\mathcal{N}_{A}\right)$, we can conclude

$$
C_{\mathfrak{F}}^{\text {exact }}\left(\mathcal{N}_{A}\right)=L R_{\mathfrak{C}}\left(\mathcal{N}_{A}\right) .
$$

\section{Exact cost under DISC}

The dephasing log-robustness is given by Eq. (117),

$L R_{\Delta}\left(\mathcal{N}_{A}\right):=D_{\max }\left(\mathcal{N}_{A} \| \Delta_{A}\left[\mathcal{N}_{A}\right]\right) \quad \forall \mathcal{N} \in \operatorname{CPTP}\left(A_{0} \rightarrow A_{1}\right)$.

By definition we have $L R_{\mathfrak{C}}\left(\mathcal{N}_{A}\right) \leqslant L R_{\Delta}\left(\mathcal{N}_{A}\right)$.

Theorem 5. The exact coherence cost of a channel $\mathcal{N}_{A} \in$ $\operatorname{CPTP}\left(A_{0} \rightarrow A_{1}\right)$ under $\mathfrak{F}=$ DISC is given by

$$
C_{\mathfrak{F}}^{\text {exact }}\left(\mathcal{N}_{A}\right)=L R_{\Delta}\left(\mathcal{N}_{A}\right) .
$$

Proof. We first prove that

$$
L R_{\Delta}\left(\mathcal{N}_{A}\right) \leqslant C_{\text {DISC }}^{0}\left(\mathcal{N}_{A}\right) \leqslant L R_{\Delta}\left(\mathcal{N}_{A}\right)+1,
$$

and then we use the additivity of $L R_{\Delta}$.

For the proof of $L R_{\Delta}\left(\mathcal{N}_{A}\right) \leqslant C_{\mathrm{DISC}}^{0}\left(\mathcal{N}_{A}\right)$, let $\Theta \in$ $\operatorname{DISC}\left(R_{1} \rightarrow A\right)$ be an optimal superchannel satisfying $\Theta_{R_{1} \rightarrow A}\left[\phi_{R_{1}}^{+}\right]=\mathcal{N}_{A}$ such that $C_{\mathrm{DISC}}^{0}\left(\mathcal{N}_{A}\right)=\log _{2}\left|R_{1}\right|$. Therefore,

$$
\begin{aligned}
L R_{\Delta}\left(\mathcal{N}_{A}\right) & =D_{\max }\left(\mathcal{N}_{A} \| \Delta_{A}\left[\mathcal{N}_{A}\right]\right) \\
& =D_{\max }\left(\Theta_{R_{1} \rightarrow A}\left[\phi_{R_{1}}^{+}\right] \| \Delta_{A} \circ \Theta_{R_{1} \rightarrow A}\left[\phi_{R_{1}}^{+}\right]\right) \\
& =D_{\max }\left(\Theta_{R_{1} \rightarrow A}\left[\phi_{R_{1}}^{+}\right] \| \Theta_{R_{1} \rightarrow A}\left[\mathcal{D}_{R_{1}}\left(\phi_{R_{1}}^{+}\right)\right]\right) \\
& \leqslant D_{\max }\left(\phi_{R_{1}}^{+} \| \mathcal{D}_{R_{1}}\left(\phi_{R_{1}}^{+}\right)\right) \\
& =\log _{2}\left|R_{1}\right| \\
& =C_{\mathrm{DISC}}^{0}\left(\mathcal{N}_{A}\right) .
\end{aligned}
$$

For the proof of $C_{\mathrm{DISC}}^{0}\left(\mathcal{N}_{A}\right) \leqslant L R_{\Delta}\left(\mathcal{N}_{A}\right)+1$, first let

$$
L R_{\Delta}\left(\mathcal{N}_{A}\right)=D_{\max }\left(\mathcal{N}_{A} \| \Delta_{A}\left[\mathcal{N}_{A}\right]\right)=\log t
$$

for some optimal $t$ that satisfies $t \Delta[\mathcal{N}] \geqslant \mathcal{N}$. Also, let $m=$ $\lceil t\rceil$ so that $m \Delta[\mathcal{N}] \geqslant \mathcal{N}$ still holds, and let $R_{1}$ be a static system with dimension $\left|R_{1}\right|=m$. We now construct the following supermap. For any state $\rho \in \mathfrak{D}\left(R_{1}\right)$,

$$
\begin{aligned}
\Omega_{R_{1} \rightarrow A}\left[\rho_{R_{1}}\right]:= & \frac{m}{m-1}\left(\operatorname{Tr}\left[\phi_{R_{1}}^{+} \rho_{R_{1}}\right]-\frac{1}{m}\right) \mathcal{N}_{A} \\
& +\frac{m}{m-1}\left(1-\operatorname{Tr}\left[\phi_{R_{1}}^{+} \rho_{R_{1}}\right]\right) \Delta_{A}\left[\mathcal{N}_{A}\right] .
\end{aligned}
$$

The supermap $\Omega_{R_{1} \rightarrow A}$ has several properties. First, it satisfies $\Delta_{A} \circ \Omega_{R_{1} \rightarrow A}=\Omega_{R_{1} \rightarrow A} \circ \mathcal{D}_{R_{1}}$. Indeed, for any density 
matrix $\rho \in \mathfrak{D}\left(R_{1}\right)$ we have

$$
\begin{aligned}
\Delta_{A} \circ \Omega_{R_{1} \rightarrow A}\left[\rho_{R_{1}}\right]= & \frac{m}{m-1}\left(\operatorname{Tr}\left[\phi_{R_{1}}^{+} \rho_{R_{1}}\right]-\frac{1}{m}\right) \Delta_{A}\left[\mathcal{N}_{A}\right] \\
& +\frac{m}{m-1}\left(1-\operatorname{Tr}\left[\phi_{R_{1}}^{+} \rho_{R_{1}}\right]\right) \Delta_{A}\left[\mathcal{N}_{A}\right] \\
= & \Delta_{A}\left[\mathcal{N}_{A}\right],
\end{aligned}
$$

and

$$
\begin{aligned}
\Omega_{R_{1} \rightarrow A} & {\left[\mathcal{D}_{R_{1}}\left(\rho_{R_{1}}\right)\right] } \\
= & \frac{m}{m-1}\left(\operatorname{Tr}\left[\phi_{R_{1}}^{+} \mathcal{D}_{R_{1}}\left(\rho_{R_{1}}\right)\right]-\frac{1}{m}\right) \mathcal{N}_{A} \\
& +\frac{m}{m-1}\left(1-\operatorname{Tr}\left[\phi_{R_{1}}^{+} \mathcal{D}_{R_{1}}\left(\rho_{R_{1}}\right)\right]\right) \Delta_{A}\left[\mathcal{N}_{A}\right] \\
= & \frac{m}{m-1}\left(\frac{1}{m}-\frac{1}{m}\right) \mathcal{N}_{A}+\frac{m}{m-1}\left(1-\frac{1}{m}\right) \Delta_{A}\left[\mathcal{N}_{A}\right] \\
= & \Delta_{A}\left[\mathcal{N}_{A}\right],
\end{aligned}
$$

so that $\Delta_{A} \circ \Omega_{R_{1} \rightarrow A}=\Omega_{R_{1} \rightarrow A} \circ \mathcal{D}_{R_{1}}$. Second, $\Omega_{R_{1} \rightarrow A}$ is a superchannel since the above map can be expressed as

$$
\begin{aligned}
\Omega_{R_{1} \rightarrow A}\left[\rho_{R_{1}}\right]:=\operatorname{Tr}[ & \left.\phi_{R_{1}}^{+} \rho_{R_{1}}\right] \mathcal{N}_{A}+\frac{1}{m-1}\left(1-\operatorname{Tr}\left[\phi_{R_{1}}^{+} \rho_{R_{1}}\right]\right) \\
& \times\left(m \Delta_{A}\left[\mathcal{N}_{A}\right]-\mathcal{N}_{A}\right)
\end{aligned}
$$

and $m \Delta_{A}\left[\mathcal{N}_{A}\right]-\mathcal{N}_{A} \geqslant 0$. Hence, $\Omega \in \operatorname{DISC}\left(R_{1} \rightarrow A\right)$. Finally, observe that $\Omega_{R_{1} \rightarrow A}\left[\phi_{R_{1}}^{+}\right]=\mathcal{N}_{A}$. Hence, the existence of such $\Omega$ implies that

$$
C_{\text {DISC }}^{0}\left(\mathcal{N}_{A}\right) \leqslant \log m=\log \lceil t\rceil \leqslant \log t+1=L R_{\Delta}\left(\mathcal{N}_{A}\right)+1 \text {. }
$$

This completes the proof.

\section{Coherence cost of a channel}

To find the approximate coherence cost (we will call it coherence cost) of any $\mathcal{N} \in \operatorname{CPTP}\left(A_{0} \rightarrow A_{1}\right)$, we first define the smoothed coherence cost as

$$
C_{\mathfrak{F}}^{\epsilon}\left(\mathcal{N}_{A}\right):=\min _{\mathcal{N}^{\prime} \in B_{\epsilon}(\mathcal{N})} C_{\mathfrak{F}}^{0}\left(\mathcal{N}_{A}^{\prime}\right),
$$

where

$$
B_{\epsilon}\left(\mathcal{N}_{A}\right)=\left\{\mathcal{N}^{\prime} \in \operatorname{CPTP}\left(A_{0} \rightarrow A_{1}\right): \frac{1}{2}\left\|\mathcal{N}_{A}^{\prime}-\mathcal{N}_{A}\right\|_{\diamond} \leqslant \epsilon\right\} .
$$

The coherence cost of the channel $\mathcal{N}_{A}$ is then given by the regularization of the smoothed coherence cost and taking the limit of $\epsilon \rightarrow 0^{+}$

$$
C_{\mathfrak{F}}\left(\mathcal{N}_{A}\right)=\lim _{\epsilon \rightarrow 0^{+}} \lim _{n \rightarrow \infty} \frac{1}{n} C_{\mathfrak{F}}^{\epsilon}\left(\mathcal{N}_{A}^{\otimes n}\right) .
$$

Below we find the coherence cost under MISC and DISC.

\section{The cost under MISC}

Theorem 6. For $\mathfrak{F}=$ MISC,

$$
C_{\mathfrak{F}}\left(\mathcal{N}_{A}\right)=L R_{\mathfrak{C}}^{\infty}\left(\mathcal{N}_{A}\right) .
$$

Proof. First, note that from Eq. (142) it follows that

$$
L R_{\mathfrak{C}^{\mathfrak{c}}}^{\epsilon}\left(\mathcal{N}_{A}\right) \leqslant C_{\mathfrak{F}}^{\epsilon}\left(\mathcal{N}_{A}\right) \leqslant L R_{\mathfrak{C}^{c}}^{\epsilon}\left(\mathcal{N}_{A}\right)+1 .
$$

Hence,

$$
\frac{1}{n} L R_{\mathfrak{C}}^{\epsilon}\left(\mathcal{N}_{A}^{\otimes n}\right) \leqslant \frac{1}{n} C_{\mathfrak{F}}^{\epsilon}\left(\mathcal{N}_{A}^{\otimes n}\right) \leqslant \frac{1}{n} L R_{\mathfrak{C}}^{\epsilon}\left(\mathcal{N}_{A}^{\otimes n}\right)+\frac{1}{n},
$$

and the limit $n \rightarrow \infty$ concludes the proof.

\section{The cost under DISC}

Theorem 7. For $\mathfrak{F}=$ DISC,

$$
C_{\mathfrak{F}}\left(\mathcal{N}_{A}\right)=L R_{\Delta}^{\infty}\left(\mathcal{N}_{A}\right) .
$$

Proof. First, note that from Eq. (156) it follows that

$$
L R_{\Delta}^{\epsilon}\left(\mathcal{N}_{A}\right) \leqslant C_{\mathfrak{F}}^{\epsilon}\left(\mathcal{N}_{A}\right) \leqslant L R_{\Delta}^{\epsilon}\left(\mathcal{N}_{A}\right)+1 .
$$

Hence,

$$
\frac{1}{n} L R_{\Delta}^{\epsilon}\left(\mathcal{N}_{A}^{\otimes n}\right) \leqslant \frac{1}{n} C_{\mathfrak{F}}^{\epsilon}\left(\mathcal{N}_{A}^{\otimes n}\right) \leqslant \frac{1}{n} L R_{\Delta}^{\epsilon}\left(\mathcal{N}_{A}^{\otimes n}\right)+\frac{1}{n},
$$

and the limit $n \rightarrow \infty$ concludes the proof.

The lack of AEP for channels motivates us to consider a more liberal method for smoothing.

\section{Liberal coherence cost of a channel}

We define the liberal one-shot $\epsilon$-approximate coherencecost as

$$
C_{\mathfrak{F}}^{\epsilon}\left(\mathcal{N}_{A}\right):=\max _{\varphi \in \mathfrak{D}\left(R A_{0}\right)} C_{\mathfrak{F}}^{\epsilon, \varphi}\left(\mathcal{N}_{A}\right),
$$

where

$$
C_{\mathfrak{F}}^{\epsilon, \varphi}\left(\mathcal{N}_{A}\right):=\min _{\mathcal{N}_{A}^{\prime} \in B_{\epsilon}^{\varphi}\left(\mathcal{N}_{A}\right)} C_{\mathfrak{F}}^{0}\left(\mathcal{N}_{A}^{\prime}\right),
$$

and

$$
\begin{aligned}
B_{\epsilon}^{\varphi}\left(\mathcal{N}_{A}\right):= & \left\{\mathcal{N}^{\prime} \in \operatorname{CP}\left(A_{0} \rightarrow A_{1}\right): \| \mathcal{N}_{A}^{\prime}\left(\varphi_{R A_{0}}\right)\right. \\
& \left.-\mathcal{N}_{A}\left(\varphi_{R A_{0}}\right) \|_{1} \leqslant \epsilon\right\} .
\end{aligned}
$$

The liberal coherence cost is defined by regularizing the liberal one-shot $\epsilon$-approximate coherence cost and then taking the limit of $\epsilon \rightarrow 0^{+}$as follows:

$$
\begin{aligned}
C_{\mathfrak{F}}^{(\infty)}\left(\mathcal{N}_{A}\right) & :=\lim _{\epsilon \rightarrow 0^{+}} \lim _{n \rightarrow \infty} \max _{\varphi \in \mathfrak{D}(R A)} \frac{1}{n} C_{\widetilde{F}}^{\epsilon, \varphi^{\otimes n}}\left(\mathcal{N}^{\otimes n}\right) \\
& =\lim _{\epsilon \rightarrow 0^{+}} \lim _{n \rightarrow \infty} \max _{\varphi \in \mathfrak{D}(R A)} \min _{\mathcal{N}^{\prime} \in B_{\epsilon}^{\varphi n}\left(\mathcal{N}^{\otimes n}\right)} \frac{1}{n} C_{\mathfrak{F}}^{0}\left(\mathcal{N}_{A^{n} \rightarrow B^{n}}^{\prime}\right) .
\end{aligned}
$$

One can interpret the above cost in the following way. For any pure state $\varphi \in \mathfrak{D}\left(R A_{0}\right)$ (with $|R|=\left|A_{0}\right|$ and $\varphi$ is full Schmidt rank) we define a $\varphi$-norm

$$
\left\|\mathcal{E}_{A}\right\|_{\varphi}:=\left\|\mathcal{E}_{A}\left(\varphi_{R A_{0}}\right)\right\|_{1} .
$$

Then the liberal cost can also be expressed as

$$
C_{\mathfrak{F}}^{(\infty)}\left(\mathcal{N}_{A}\right)=\lim _{\epsilon \rightarrow 0^{+}} \lim _{n \rightarrow \infty} \max _{\varphi \in \mathfrak{D}\left(R A_{0}\right)} \min _{\left\|\mathcal{N}^{\prime}-\mathcal{N}^{\otimes n}\right\|_{\varphi} \otimes n} \frac{1}{n} C_{\mathfrak{F}}^{0}\left(\mathcal{N}_{A^{n} \rightarrow B^{n}}^{\prime}\right) .
$$

That is, we smooth with the $\varphi_{R A_{0}}^{\otimes n}$-norm and then maximizing over all such norms. 
Theorem 8. For $\mathfrak{F}=$ MISC,

$$
C_{\mathfrak{F}}^{(\infty)}\left(\mathcal{N}_{A}\right)=D_{\mathfrak{C}}^{(\infty)}\left(\mathcal{N}_{A}\right) .
$$

Proof. From Eq. (142) it follows that that for any fixed $\varphi \in$ $\mathfrak{D}\left(R A_{0}\right)$ we have

$$
L R_{\mathfrak{C}}^{\epsilon, \varphi}\left(\mathcal{N}_{A}\right) \leqslant C_{\mathfrak{F}}^{\epsilon, \varphi}\left(\mathcal{N}_{A}\right) \leqslant L R_{\mathfrak{C}}^{\epsilon, \varphi}\left(\mathcal{N}_{A}\right)+1 .
$$

From Eq. (185) it follows that $C_{\mathfrak{F}}^{(\infty)}\left(\mathcal{N}_{A}\right)=L R_{\mathfrak{C}}^{(\infty)}\left(\mathcal{N}_{A}\right)$ so that the theorem follows from the AEP relation Eq. (133).

\section{E. One-shot distillable coherence}

We now consider the problem of distilling an arbitrary channel into pure-state coherence using MISC and DISC. Let $\Theta \in \mathfrak{F}\left(A \rightarrow B_{1}\right)$ where $\mathfrak{F}=$ MISC or DISC, such that for any input channel $\mathcal{E}_{A}$, the output is a state preparation channel $\mathcal{F}_{B} \in \operatorname{CPTP}\left(B_{0} \rightarrow B_{1}\right)$ where $B_{0}$ is a trivial system. For $\epsilon>0$ and $n=\left|B_{1}\right|$, define

$$
\begin{gathered}
\operatorname{DISTILL}_{\mathfrak{F}}^{\epsilon}\left(\mathcal{N}_{A}\right)=\log \max \left\{n:\left\langle\phi_{B_{1}}^{+}\left|\Theta\left[\mathcal{N}_{A}\right]\right| \phi_{B_{1}}^{+}\right\rangle>1-\epsilon,\right. \\
\left.\Theta \in \mathfrak{F}\left(A \rightarrow B_{1}\right)\right\},
\end{gathered}
$$

which represents the largest coherence attainable by MISC or DISC within $\epsilon$-error. For all $\mathcal{N} \in \operatorname{CPTP}\left(A_{0} \rightarrow A_{1}\right)$, we can write

$$
\begin{aligned}
\left\langle\phi_{B_{1}}^{+}|\Theta[\mathcal{N}]| \phi_{B_{1}}^{+}\right\rangle & =\left\langle\phi_{B_{1}}^{+}\left|\left(\operatorname{Tr}_{A}\left[\mathbf{J}_{A B_{1}}^{\Theta}\left(\left(J_{A}^{\mathcal{N}}\right)^{T} \otimes I_{B_{1}}\right)\right]\right)\right| \phi_{B_{1}}^{+}\right\rangle \\
& =\operatorname{Tr}\left[\mathbf{J}_{A B_{1}}^{\Theta}\left(\left(J_{A}^{\mathcal{N}}\right)^{T} \otimes \phi_{B_{1}}^{+}\right)\right] .
\end{aligned}
$$

Note that the space of all operators that are invariant under any permutation in the classical basis, is a linear combination of maximally mixed state, $u_{A_{1}}$ and maximally coherent state, $\phi_{A_{1}}^{+}$. Any operator is permutation invariant if

$$
\Pi_{x} \sigma \Pi_{x}^{\dagger}=\sigma \quad \forall \text { permutation matrices } \Pi_{x} \text {. }
$$

The permutation-twirling operation can be expressed in the following way (see, for example, Ref. [89]):

$$
\mathcal{T}(\cdot)=\frac{1}{m !} \sum_{x} \Pi_{x}(\cdot) \Pi_{x}^{\dagger} \forall \Pi_{x},
$$

where $m$ is the dimension of the input system. Observe that the output of the above permutation-twirling operation on any state is permutation invariant and so can always be represented as a linear combination of $\phi_{A_{1}}^{+}$and $u_{A_{1}}$. Hence, we can express the second equality in Eq. (187) as

$$
\begin{aligned}
\operatorname{Tr} & {\left[\mathbf{J}_{A B_{1}}^{\Theta}\left(\left(J_{A}^{\mathcal{N}}\right)^{T} \otimes \phi_{B_{1}}^{+}\right)\right] } \\
& =\operatorname{Tr}\left[\mathbf{J}_{A B_{1}}^{\Theta}\left(\left(J_{A}^{\mathcal{N}}\right)^{T} \otimes \mathcal{T}\left(\phi_{B_{1}}^{+}\right)\right)\right] \\
& =\operatorname{Tr}\left[\left(\mathrm{id}_{A} \otimes \mathcal{T}\left(\mathbf{J}_{A B_{1}}^{\Theta}\right)\right)\left(\left(J_{A}^{\mathcal{N}}\right)^{T} \otimes \phi_{B_{1}}^{+}\right)\right],
\end{aligned}
$$

where the second equality follows from the fact that $\mathcal{T}$ is selfadjoint in the Hilbert-Schmidt inner product. Hence, without loss of generality we can express the Choi matrix $\mathbf{J}_{A B_{1}}^{\Theta}$ in following way

$$
\mathbf{J}_{A B_{1}}^{\Theta}=\alpha_{A} \otimes \phi_{B_{1}}^{+}+\frac{1}{n-1} \beta_{A} \otimes\left(I_{B_{1}}-\phi_{B_{1}}^{+}\right),
$$

where $n=\left|B_{1}\right|$ and $\alpha_{A}, \beta_{A} \in \operatorname{Herm}(A)$ such that $\mathbf{J}_{A B_{1}}^{\Theta} \geqslant 0$, $\mathbf{J}_{A_{1}}^{\Theta}=I_{A_{1}}$, and $\mathbf{J}_{A}^{\Theta}=\mathbf{J}_{A_{0}}^{\Theta} \otimes u_{A_{1}}$. In terms of $\alpha_{A}$ and $\beta_{A}$, we can write these conditions as

$$
\begin{aligned}
\alpha_{A}, \beta_{a} & \geqslant 0, \\
\operatorname{Tr}\left(\alpha_{A}+\beta_{A}\right) & =\left|A_{1}\right|, \\
\alpha_{A}+\beta_{A} & =\operatorname{Tr}_{A_{1}}\left(\alpha_{A}+\beta_{A}\right) \otimes u_{A_{1}} .
\end{aligned}
$$

From the MISC condition of $\mathcal{D}_{A B}\left(\mathbf{J}_{A B}^{\Theta}\right)=\mathcal{D}_{A} \otimes \operatorname{id}_{B}\left(\mathbf{J}_{A B}^{\Theta}\right)$, we get

$$
\mathcal{D}\left(\alpha_{A}\right)(n-1)=\mathcal{D}\left(\beta_{A}\right) .
$$

Defining $\beta_{A}=\rho_{A_{0}} \otimes I_{A_{1}}-\alpha_{A}$ where $\rho_{A_{0}}=\frac{1}{\left|A_{1}\right|} \operatorname{Tr}_{A_{1}}\left(\alpha_{A}+\right.$ $\left.\beta_{A}\right)$. Since $\operatorname{Tr}\left[\rho_{A_{0}}\right]=1, \rho_{A_{0}}$ is a density matrix. So, we can rewrite these constraints as

$$
\begin{aligned}
\alpha_{A} & \geqslant 0, \\
\rho_{A_{0}} \otimes I_{A_{1}} & \geqslant \alpha_{A}, \\
\frac{1}{n} \mathcal{D}\left(\rho_{A_{0}}\right) \otimes I_{A_{1}} & =\mathcal{D}\left(\alpha_{A}\right), \\
\rho_{A_{0}} & \in \mathfrak{D}\left(A_{0}\right) .
\end{aligned}
$$

We can also consider imposing the additional DISC constraint of $\mathrm{id}_{A} \otimes \mathcal{D}_{B}\left(\mathbf{J}_{A B}^{\Theta}\right)=\mathcal{D}_{A} \otimes \operatorname{id}_{B}\left(\mathbf{J}_{A B}^{\Theta}\right)$ which gives

$$
\alpha_{A}+\beta_{A}=\mathcal{D}\left(\alpha_{A}+\beta_{A}\right) .
$$

This amounts to replacing Eq. (197) with the condition

$$
n \mathcal{D}\left(\alpha_{A}\right) \geqslant \alpha_{A} \text {. }
$$

Next notice that we can always write $\alpha_{A}=\mathcal{D}_{A}\left(\alpha_{A}\right)+\gamma_{A}$ for some $\gamma_{A}$ with zeros on the diagonal. Then, since $\operatorname{Tr}_{A_{1}}\left[\left(J_{A}^{\mathcal{N}}\right)^{T}\right]=I_{A_{0}}$, we can write

$$
\begin{aligned}
\operatorname{Tr} & {\left[\alpha_{A}\left(J_{A}^{\mathcal{N}}\right)^{T}\right] } \\
& =\operatorname{Tr}\left[\left(\mathcal{D}_{A}\left(\alpha_{A}\right)+\gamma_{A}\right)\left(J_{A}^{\mathcal{N}}\right)^{T}\right] \\
& =\operatorname{Tr}\left[\mathcal{D}_{A}\left(\alpha_{A}\right)\left(J_{A}^{\mathcal{N}}\right)^{T}\right]+\operatorname{Tr}\left[\gamma_{A}\left(J_{A}^{\mathcal{N}}\right)^{T}\right] \\
& =\operatorname{Tr}\left[\left(\frac{1}{n} \mathcal{D}\left(\rho_{A_{0}}\right) \otimes I_{A_{1}}\right)\left(J_{A}^{\mathcal{N}}\right)^{T}\right]+\operatorname{Tr}\left[\gamma_{A}\left(J_{A}^{\mathcal{N}}\right)^{T}\right] \\
& =\frac{1}{n}+\operatorname{Tr}\left[\gamma_{A}\left(J_{A}^{\mathcal{N}}\right)^{T}\right] .
\end{aligned}
$$

Hence, we have the following one-shot distillable rates.

Theorem 9. For $\mathfrak{F}=$ MISC or DISC,

$$
\operatorname{DISTILL}_{\mathfrak{F}}^{\epsilon}(\mathcal{N})=\log \max n,
$$

such that

$$
\begin{gathered}
\operatorname{Tr}\left[\gamma_{A}\left(J_{A}^{\mathcal{N}}\right)^{T}\right] \geqslant 1-\frac{1}{n}-\epsilon, \\
\mathcal{D}_{A}\left(\gamma_{A}\right)=0, \\
\rho_{A_{0}} \in \mathfrak{D}\left(A_{0}\right),
\end{gathered}
$$




$$
\begin{gathered}
{\left[\rho_{A_{0}}-\frac{1}{n} \mathcal{D}_{A_{0}}\left(\rho_{A_{0}}\right)\right] \otimes I_{A_{1}} \geqslant \gamma_{A} \geqslant-\frac{1}{n} \mathcal{D}_{A_{0}}\left(\rho_{A_{0}}\right) \otimes I_{A_{1}} \quad \text { (specifically for } \mathfrak{F}=\text { MISC) },} \\
\frac{n-1}{n} \mathcal{D}_{A_{0}}\left(\rho_{A_{0}}\right) \otimes I_{A_{1}} \geqslant \gamma_{A} \geqslant-\frac{1}{n} \mathcal{D}_{A_{0}}\left(\rho_{A_{0}}\right) \otimes I_{A_{1}} \quad \text { (specifically for } \mathfrak{F}=\text { DISC) } .
\end{gathered}
$$

Remark 1. The first condition in the above theorem, i.e., Eq. (204) follows by substituting Eq. (191) in $\operatorname{Tr}\left[\mathbf{J}_{A B_{1}}^{\Theta}\left(\left(J_{A}^{\mathcal{N}}\right)^{T} \otimes \phi_{B_{1}}^{+}\right)\right]$and using the fact that $\operatorname{Tr}\left[\phi_{A_{1}}^{+} \phi_{A_{1}}^{+}\right]=$ $\operatorname{Tr}\left[\phi_{A_{1}}^{+}\right]$, and then plugging the result in Eq. (202). The 1.h.s. of Eq. (207) is a consequence of Eq. (197) and (198) whereas the r.h.s. is a consequence of Eqs. (196) and (198). Similarly, for DISC, the 1.h.s. of Eq. (208) is a consequence of Eqs. (198) and (201) whereas the r.h.s. of Eq. (208) is a consequence of Eqs. (196) and (198).

Remark 2. Note that $D_{\text {MISC }}^{\epsilon}(\mathcal{N})=D_{\text {DISC }}^{\epsilon}(\mathcal{N})$ when $\left|A_{0}\right|=$ 1 , and their common rate matches that given in Refs. [90,91] for distilling coherence from static resources (i.e., states). However for channels, the MISC and DISC distillable coherence can possibly differ. We leave it as an open problem to find channels that have such a property.

Example 1. Let us consider the partially depolarizing channel $\mathcal{N}_{\lambda, d}^{\text {dep }}: \mathcal{B}\left(A_{1}\right) \rightarrow \mathcal{B}\left(A_{1}\right)$,

$$
\mathcal{N}_{\lambda, d}^{\mathrm{dep}}(\chi)=\lambda \chi+(1-\lambda) \operatorname{Tr}[\chi] u_{A_{1}},
$$

where $d=\left|A_{1}\right|$. The Choi matrix of this channel is given by

$$
J_{A_{1} \tilde{A}_{1}}^{\mathcal{N}^{\mathrm{dep}}}=\lambda \phi_{A_{1} \tilde{A}_{1}}^{+}+\frac{1-\lambda}{d} I_{A_{1} \tilde{A}_{1}} .
$$

We exploit the symmetry by noting that both $\phi_{A_{1} \tilde{A}_{1}}^{+}$and $I_{A_{1} \tilde{A}_{1}}$ are $U^{*} \otimes U$ invariant. We restrict our twirling to an average over the group of incoherent unitaries, i.e., each $U$ involves a permutation and/or a change in relative phase. Note that dephasing commutes with this operation so if Eq. (198) holds before the twirl, it will also hold after. The action of twirling will convert $\rho_{A_{1}} \otimes I_{\tilde{A}_{1}} \rightarrow u_{A_{1}} \otimes I_{\tilde{A}_{1}}$ while converting $\alpha_{A}$ into an operator of the form

$$
\begin{aligned}
\alpha_{A_{1} \tilde{A}_{1}} & =p \sum_{i \neq j}|i j\rangle\left\langle i j\left|+q \sum_{i}\right| i i\right\rangle\left\langle i i\left|+r \sum_{i \neq j}\right| i i\right\rangle\langle j j| \\
& =p \sum_{i \neq j}|i j\rangle\left\langle i j\left|+(q-r) \sum_{i}\right| i i\right\rangle\langle i i|+r \phi_{A_{1} \tilde{A}_{1}}^{+} .
\end{aligned}
$$

The eigenvalues of $\alpha_{A_{1} \tilde{A}_{1}}$ are easily seen to be $\{p, q-r, q-$ $r+r d\}$, and so Eqs. (196) and (197) require that $p, q-r \geqslant 0$ and $p, q-r+r d \leqslant \frac{1}{d}$. From Eq. (198), we must also have $p=q=\frac{1}{n d}$. With these constraints in place, our goal is to maximize $n$ such that

$$
\begin{aligned}
\operatorname{Tr}\left[\alpha_{A_{1} \tilde{A}_{1}}^{T} J_{A_{1} \tilde{A}_{1}}^{\mathcal{N}_{\text {dep }}}\right]= & (1-\lambda) \frac{(d-1)}{n d}+\left(\frac{1}{n d}-r\right) \\
& \times[\lambda d+(1-\lambda)]+r\left[\lambda d^{2}+(1-\lambda)\right] .
\end{aligned}
$$

This function is strictly increasing w.r.t. $r$, and the constraints necessitate that $r \leqslant \min \left\{\frac{n-1}{d-1} \frac{1}{n d}, \frac{1}{n d}\right\}$. So when $n \leqslant d$, we take $r=\frac{n-1}{d-1} \frac{1}{n d}$ and obtain

$$
\begin{aligned}
\operatorname{Tr}\left[\alpha_{A_{1} \tilde{A}_{1}}^{T} J_{A_{1} \tilde{A}_{1}}^{\mathcal{N}_{\mathrm{dep}}}\right]= & (1-\lambda) \frac{d-1}{n d}+\frac{d-n}{n d(d-1)}[\lambda d+(1-\lambda)] \\
& +\frac{n-1}{n d(d-1)}\left[\lambda d^{2}+(1-\lambda)\right] \\
= & (1-\lambda) \frac{1}{n}+\lambda .
\end{aligned}
$$

Notice that when $\lambda=1$ we obtain $\operatorname{Tr}\left[\alpha_{A_{1} \tilde{A}_{1}}^{T} J_{A_{1} \tilde{A}_{1}}^{\mathcal{N}^{\text {dep }}}\right]=1$. This says that $\log n$ bits can be perfectly distilled, which is expected: the free superchannel just consists of inputting $\phi_{A_{1} \tilde{A}_{1}}^{+}$ into the given channel and then as post-processing performs a MIO map that converts $\phi_{A_{1} \tilde{A}_{1}}^{+}$into $\phi_{B_{1}}^{+}$. However, if $n \geqslant d$, then we take $r=\frac{1}{n d}$ and Eq. (213) becomes

$$
\operatorname{Tr}\left[\alpha_{A_{1} \tilde{A}_{1}}^{T} J_{A_{1} \tilde{A}_{1}}^{\mathcal{N}_{\text {dep }}}\right]=(1-\lambda) \frac{1}{n}+\frac{d}{n} \lambda .
$$

Notice also that in this case our optimizer $\rho_{A_{0}}$ is completely dephased, which means our solution for MISC is also the solution for DISC. We summarize our findings as follows.

Lemma 7. For the partial depolarizing channel $\mathcal{N}_{\lambda, d}^{\text {dep }}$ and $0 \leqslant \epsilon<1$,

$$
\begin{aligned}
\operatorname{DISTILL}_{\mathrm{MISC}}^{\epsilon}\left(\mathcal{N}_{\lambda, d}^{\mathrm{dep}}\right) & =\operatorname{DISTILL}_{\mathrm{DISC}}^{\epsilon}\left(\mathcal{N}_{\lambda, d}^{\text {dep }}\right) \\
& = \begin{cases}\log \left\lfloor\frac{1-\lambda}{1-\lambda-\epsilon}\right\rfloor & \text { if } \epsilon<\frac{(d-1)(1-\lambda)}{d} \\
\log \left\lfloor\frac{1-\lambda+\lambda d}{1-\epsilon}\right\rfloor & \text { if } \epsilon \geqslant \frac{(d-1)(1-\lambda)}{d}\end{cases}
\end{aligned}
$$

Example 2. We next consider the partial dephasing chan$\operatorname{nel} \mathcal{N}_{\lambda, d}^{\Delta}: \mathcal{B}\left(A_{1}\right) \rightarrow \mathcal{B}\left(A_{1}\right)$,

$$
\mathcal{N}_{\lambda, d}^{\Delta}(\chi)=\lambda \chi+(1-\lambda) \mathcal{D}(\chi)
$$

The Choi matrix of this channel is given by

$$
J_{A_{1} \tilde{A}_{1}}^{\mathcal{N}^{\text {dep }}}=\lambda \phi_{A_{1} \tilde{A}_{1}}^{+}+(1-\lambda) \sum_{i=1}^{d}|i i\rangle\langle i i| .
$$

By the same argument as before, we can assume without loss of generality that $\alpha_{A}$ has the form

$$
\alpha_{A_{1} \tilde{A}_{1}}=p \sum_{i \neq j}|i j\rangle\left\langle i j\left|+(q-r) \sum_{i}\right| i i\right\rangle\langle i i|+r \phi_{A_{1} \tilde{A}_{1}}^{+} .
$$

However, this time the fidelity with $\phi_{B_{1}}^{+}$is given by

$$
\operatorname{Tr}\left[\alpha_{A_{1} \tilde{A}_{1}}^{T} J_{A_{1} \tilde{A}_{1}}^{\mathcal{N}^{\Delta}}\right]=\left(\frac{1}{n d}-r\right) d+r\left[\lambda d^{2}+(1-\lambda) d\right]
$$


Again, the constraints of the problem demand $r \leqslant$ $\min \left\{\frac{n-1}{d-1} \frac{1}{n d}, \frac{1}{n d}\right\}$. When $n \leqslant d$, it holds that

$$
\begin{aligned}
\operatorname{Tr}\left[\alpha_{A_{1} \tilde{A}_{1}}^{T} J_{A_{1} \tilde{A}_{1}}^{\mathcal{N}^{\Delta}}\right] & =\frac{d-n}{n(d-1)}+\frac{n-1}{n(d-1)}[\lambda d+(1-\lambda)] \\
& =\frac{1+(n-1) \lambda}{n} .
\end{aligned}
$$

However, when $n \geqslant d$, we take $r=\frac{1}{n d}$ to obtain

$$
\operatorname{Tr}\left[\alpha_{A_{1} \tilde{A}_{1}}^{T} J_{A_{1} \tilde{A}_{1}}^{\mathcal{N}}\right]=\frac{\lambda d+(1-\lambda)}{n} .
$$

These are the same maximum fidelities as the depolarizing channel, and we therefore have the following conclusion.

Lemma 8. For the partial dephasing channel $\mathcal{N}_{\lambda, d}^{\Delta}$ and $0 \leqslant$ $\epsilon<1$,

$$
\begin{aligned}
\operatorname{DISTILL}_{\mathrm{MISC}}^{\epsilon}\left(\mathcal{N}_{\lambda, d}^{\Delta}\right) & =\operatorname{DISTILL}_{\mathrm{DISC}}^{\epsilon}\left(\mathcal{N}_{\lambda, d}^{\Delta}\right) \\
& = \begin{cases}\log \left\lfloor\frac{1-\lambda}{1-\lambda-\epsilon}\right\rfloor & \text { if } \epsilon<\frac{(d-1)(1-\lambda)}{d} \\
\log \left\lfloor\frac{1-\lambda+\lambda d}{1-\epsilon}\right\rfloor & \text { if } \epsilon \geqslant \frac{(d-1)(1-\lambda)}{d}\end{cases}
\end{aligned}
$$

\section{OUTLOOK AND CONCLUSIONS}

In this paper, we have developed the resource theory of dynamical coherence using the classical channels as free channels. In previous works on the quantum resource theory of dynamical coherence [27,29,30,35,43], the free channels were taken to be the free operations from the QRT of static coherence, like MIO, IO, etc. However, if we consider distributed quantum computing scenarios, then one encounters channels with noisy pre- and post-processing links. In this case, a natural candidate for free channels are those with completely dephasing pre- and post- processing. What emerges is a resource theory in which the free objects are the set of classical channels. Note that in such a theory, the T-gate (crucial for quantum computation) is not free and even the quantum identity channel is not free as the preservation of coherence should be considered a resource.

Similar to the static QRT of coherence where the free operations can have a nonfree dilation, in our work on dynamical QRT of coherence, the free superchannels can have a nonfree realization. That means, the pre- and post-processing channels need not be classical. The only requirement on the set of free superchannels comes from the golden rule of QRT. This implies that the free superchannels must never generate coherent channels when the input channels are classical, even when tensored with identity, i.e., even when the free superchannel acts on a part of the input classical channel. This enlargement of the set of free superchannels is necessary for a meaningful resource theory of coherence. Take for example the set of free superchannels which can be realized only by classical pre- and post-processing channels. In this case, the output channel is always classical irrespective of the input channel, eliminating all the advantage offered by a quantum channel. Thus, such free superchannels cannot be used to study the resource theory of quantum coherence.

In Sec. III, we start by defining four sets of free superchannels. We name them as maximally incoherent superchannels (MISC), dephasing-covariant incoherent superchannels
(DISC), incoherent superchannels (ISC), and strictly incoherent superchannels (SISC). We show that the set of free superchannels in the dynamical resource theory of coherence can be characterized analogous to the free channels in the static resource theory of coherence. We also show that MISC and DISC can be characterized just on the basis of their Choi matrices and dephasing channels which is given in Eqs. (76) and (84) for MISC and DISC, respectively.

Section IV then deals with the quantification of dynamical coherence. In Sec. IV A, we find the complete set of monotones for MISC and DISC. That means, to see if we can convert from one quantum channel to another, it is sufficient to check if all the monotones of this (complete) set acting on one channel are greater than the other. A complete family of monotones for a general resource theory of processes was presented in [51]. It is, in general, a hard problem to compute these functions and in some cases like LOCC-based entanglement, it is even NP-hard. We show that for the resource theory of dynamical coherence, these functions (under MISC and DISC) can be computed using an SDP [Eq. (92)]. Next, in Sec. IV B, we also find monotones that are based on the relative entropy. In Ref. [45], Gour and Winter showed that the generalization of relative entropy from states to channels is not unique. In their work, they listed six relative entropies as measures of dynamical resources. They also introduced a new type of smoothing called "liberal" smoothing. We show in Sec. IV B that out of these relative entropies defined in Ref. [45], three relative entropies clearly form a monotone under MISC. For the case of DISC, we show that the channel divergence for a given channel and the same channel acted on by the dephasing superchannel forms a resource monotone under DISC. We then discuss various types of channel log-robustness of coherence, which are based on the maxrelative entropy of channels $D_{\max }$, and we show that it can be computed with an SDP [Eq. (107)]. For the qubit case, we calculated the log-robustness of coherence for classical channels, identity channel, replacement channel, depolarizing channels, and unitary channels. We also show that the logrobustness of coherence of channels is additive under tensor product (Lemma 4). We then define a "liberally" smoothed log-robustness of coherence which when regularized is equal to a regularized relative entropy introduced in [45] (i.e., it satisfies AEP), and behaves monotonically under completely resource nongenerating superchannels.

The next section is dedicated to the problem of interconversion of one resource into another. In Sec. V A, we define a conversion distance between two channels [Eq. (134)]. A given channel can be simulated using another if the interconversion distance is very small. For MISC and DISC, we showed that the interconversion distance can be computed using an SDP (Theorem 3). We then calculated the exact, asymptotic, and liberal cost of coherence of a channel and found that the liberal cost of coherence is equal to a variant of regularized relative entropy. Last, in this section, we also define the one-shot distillable coherence for MISC and DISC, and calculate it for partial depolarizing and partial dephasing channels.

Due to the realization of a superchannel as a pre- and postprocessing channel, there are added complexities in the generalization of a quantum resource theory of states to channels as mentioned in Ref. [51]. In our case, we see that the simple 
generalizations do not work. For example, while calculating coherence costs, we had to introduce the concept of liberal cost (based on liberal smoothing as defined in Ref. [45]) to show it to be equal to a relative entropy.

Clearly, our work is just a start of a whole unexplored field of the quantum resource theory of dynamical coherence. For instance, one can solve for interconversion, cost, etc., for ISC and SISC. One can define more sets of superchannels analogous to how various free operations are defined in the static case. We also leave as open the problem of finding an example of a channel where the MISC and DISC distillable coherence are different. In Sec. VE, we worked out the distillable coherence for the partial depolarizing channel and the partial dephasing channel and found no difference for MISC and DISC case.

Note added. Recently, we became aware of the work [92] which considers resource preserving channels as a resource in a general resource theory.

\section{ACKNOWLEDGMENTS}

The authors thank Karol Horodecki, Varun Narasimhachar, Yunlong Xiao, and Carlo Maria Scandolo for helpful discussions. G.G. and G.S. acknowledge support from the Natural Sciences and Engineering Research Council of Canada (NSERC). E.C. is supported by National Science Foundation (NSF) Award No. 1820871.

\section{APPENDIX A: PROOF OF DUAL OF THE LOG-ROBUSTNESS}

Finding the dual of the log-robustness $\left[L R_{\mathfrak{C}}\left(\mathcal{N}_{A}\right)\right]$ is equivalent to finding the dual of $2^{L R_{\mathfrak{C}}\left(\mathcal{N}_{A}\right)}$. From Eq. (107), we can write $2^{L R_{\mathfrak{C}}\left(\mathcal{N}_{A}\right)}$ as

$$
\begin{aligned}
& \min \left\{\frac{1}{\left|A_{0}\right|} \operatorname{Tr}\left[\omega_{A}\right]: \omega_{A} \geqslant J_{A}^{\mathcal{N}}, \quad \mathcal{D}_{A}\left[\omega_{A}\right]=\omega_{A},\right. \\
& \left.\omega_{A_{0}}=\operatorname{Tr}\left[\omega_{A}\right] u_{A_{0}}, \omega_{A} \geqslant 0\right\},
\end{aligned}
$$

where $u_{A_{0}}=\frac{I_{A_{0}}}{\left|A_{0}\right|}$. The primal problem of the above conic linear program can be stated as

$$
\min \left\{\frac{1}{\left|A_{0}\right|} \operatorname{Tr}\left[\omega_{A} I_{A}\right]: \Gamma\left(\omega_{A}\right)-H_{2} \in \mathfrak{K}_{2}, \omega \geqslant 0\right\},
$$

where $\Gamma\left(\omega_{A}\right)$ is a linear map and is expressed as a 3-tuple such that $\Gamma\left(\omega_{A}\right)=\left\{\omega_{A_{0}}-\operatorname{Tr}\left[\omega_{A_{0}}\right] u_{A_{0}}, \omega_{A}, \omega_{A}-\right.$ $\left.\mathcal{D}\left(\omega_{A}\right)\right\}$. The separation of elements in the tuple can be understood as a direct sum between the subspaces in a larger vector space. Likewise, $H_{2}$ is also expressed as a 3tuple such that $H_{2}=\left(0_{A_{0}}, J_{A}^{\mathcal{N}}, 0_{A}\right)$. The cone $\mathfrak{K}_{2}$ can be expressed as a 3 -tuple as $\mathfrak{K}_{2}=\left\{\left(0_{A_{0}}, \zeta_{A}, 0_{A}\right): \zeta_{A} \geqslant 0\right\}$. Hence, the dual cone $\mathfrak{K}_{2}^{*}=\left\{\left(Z_{A_{0}}, \beta_{A}, W_{A}\right): Z_{A_{0}} \in \operatorname{Herm}\left(A_{0}\right), \beta_{A} \geqslant\right.$ $\left.0, W_{A} \in \operatorname{Herm}(A)\right\}$.

Therefore, it is easy to see that the dual to the above primal problem is

$$
\begin{gathered}
\max \left\{\frac{1}{\left|A_{0}\right|} \operatorname{Tr}\left[\beta_{A} J_{A}^{\mathcal{N}}\right]: I_{A}-\Gamma^{*}\left(Z_{A_{0}}, \beta_{A}, W_{A}\right) \geqslant 0,\right. \\
\left.Z_{A_{0}} \in \operatorname{Herm}\left(A_{0}\right), W_{A} \in \operatorname{Herm}(A), \beta_{A} \geqslant 0\right\} .
\end{gathered}
$$

To find $\Gamma^{*}\left(Z_{A_{0}}, \beta_{A}, W_{A}\right)$, we need to equate

$$
\operatorname{Tr}\left[\left(Z_{A_{0}}, \beta_{A}, W_{A}\right) \Gamma\left(\omega_{A}\right)\right]=\operatorname{Tr}\left[\Gamma^{*}\left(Z_{A_{0}}, \beta_{A}, W_{A}\right) \omega_{A}\right] .
$$

From the 1.h.s. of Eq. (A4), we find

$$
\begin{aligned}
\operatorname{Tr} & {\left[\left(Z_{A_{0}}, \beta_{A}, W_{A}\right) \Gamma\left(\omega_{A}\right)\right] } \\
= & \operatorname{Tr}\left[Z_{A_{0}}\left(\omega_{A_{0}}-\operatorname{Tr}\left[\omega_{A_{0}}\right] u_{A_{0}}\right)\right] \\
& +\operatorname{Tr}\left[\beta_{A} \omega_{A}\right]+\operatorname{Tr}\left[W_{A}\left(\omega_{A}-\mathcal{D}\left(\omega_{A}\right)\right)\right] .
\end{aligned}
$$

Therefore,

$$
\begin{aligned}
\Gamma^{*}\left(Z_{A_{0}}, \beta_{A}, W_{A}\right)= & Z_{A_{0}} \otimes I_{A_{1}}-\operatorname{Tr}\left[Z_{A_{0}}\right] u_{A_{0}} \otimes I_{A_{1}}+\beta_{A} \\
& +W_{A}-\mathcal{D}\left(W_{A}\right) .
\end{aligned}
$$

So, we can rewrite the first constraint in the dual problem as

$$
\begin{aligned}
& \frac{I_{A}}{\left|A_{0}\right|}-Z_{A_{0}} \otimes I_{A_{1}}+\operatorname{Tr}\left[Z_{A_{0}}\right] u_{A_{0}} \\
& \quad \otimes I_{A_{1}}-\beta_{A}-W_{A}+\mathcal{D}\left(W_{A}\right) \geqslant 0 .
\end{aligned}
$$

Now let $\eta_{A} \geqslant 0$ obey the following conditions:

$$
\mathcal{D}_{A}\left(\eta_{A}\right)=\mathcal{D}_{A_{0}}\left(\eta_{A_{0}}\right) \otimes u_{A_{1}}, \quad \mathcal{D}_{A_{1}}\left[\eta_{A_{1}}\right]=I_{A_{1}} .
$$

Any such matrix can be expressed as $\left(u_{A_{0}}-Z_{A_{0}}+\operatorname{Tr}\left[Z_{A_{0}}\right] u_{A_{0}}\right) \otimes I_{A_{1}}-W_{A}+\mathcal{D}\left(W_{A}\right)$. Hence, we can express Eq. (A7) as

$$
\eta_{A} \geqslant \beta_{A} \geqslant 0 .
$$

Since $J_{A}^{\mathcal{N}} \geqslant 0$, therefore from the above equation we get

$$
\operatorname{Tr}\left[\eta_{A} J_{A}^{\mathcal{N}}\right] \geqslant \operatorname{Tr}\left[\beta_{A} J_{A}^{\mathcal{N}}\right] .
$$

Hence, we can recast the dual problem in the following form:

$$
\begin{aligned}
& \max \left\{\operatorname{Tr}\left[\eta_{A} J_{A}^{\mathcal{N}}\right]: \mathcal{D}_{A}\left(\eta_{A}\right)=\mathcal{D}_{A_{0}}\left(\eta_{A_{0}}\right) \otimes u_{A_{1}},\right. \\
& \left.\mathcal{D}_{A_{1}}\left[\eta_{A_{1}}\right]=I_{A_{1}}, \quad \eta_{A} \geqslant 0\right\} .
\end{aligned}
$$

Therefore,

$$
\begin{aligned}
L R_{\mathfrak{C}}\left(\mathcal{N}_{A}\right)= & \log \max \left\{\operatorname{Tr}\left[\eta_{A} J_{A}^{\mathcal{N}}\right]: \mathcal{D}_{A}\left(\eta_{A}\right)=\mathcal{D}_{A_{0}}\left(\eta_{A_{0}}\right) \otimes u_{A_{1}},\right. \\
& \left.\mathcal{D}_{A_{1}}\left[\eta_{A_{1}}\right]=I_{A_{1}}, \quad \eta_{A} \geqslant 0\right\},
\end{aligned}
$$

which is Eq. (108).

\section{APPENDIX B: PROOF OF THEOREM 3 AND THE DUAL OF THE CONVERSION DISTANCE FOR MISC AND DISC}

In Ref. [87], it was shown that the diamond norm can be expressed as the following SDP:

$$
\begin{aligned}
& \frac{1}{2}\left\|\mathcal{E}_{B}-\mathcal{F}_{B}\right\|_{\diamond} \\
& =\min _{\omega \geqslant 0 ; \omega \geqslant J_{B}^{\mathcal{E}-\mathcal{F}}}\left\|\omega_{B_{0}}\right\|_{\infty} \forall \mathcal{E}, \mathcal{F} \in \operatorname{CPTP}\left(B_{0} \rightarrow B_{1}\right) .
\end{aligned}
$$

Note that Eq. (B1) can be rewritten as [45]

$$
\begin{aligned}
\frac{1}{2}\left\|\mathcal{E}_{B}-\mathcal{F}_{B}\right\|_{\diamond}= & \min \left\{\lambda: \lambda \mathcal{Q}_{B} \geqslant \mathcal{E}_{B}-\mathcal{F}_{B} ;\right. \\
& \left.\mathcal{Q}_{B} \in \operatorname{CPTP}\left(B_{0} \rightarrow B_{1}\right)\right\} .
\end{aligned}
$$

Taking $\mathcal{E}_{B}=\Theta_{A \rightarrow B}\left[\mathcal{N}_{A}\right]$ and $\mathcal{F}_{B}=\mathcal{M}_{B}, d_{\mathfrak{F}}\left(\mathcal{N}_{A} \rightarrow \mathcal{M}_{B}\right)$ in Eq. (134) becomes

$$
\begin{aligned}
d_{\mathfrak{F}}\left(\mathcal{N}_{A} \rightarrow \mathcal{M}_{B}\right)= & \min \left\{\lambda: \lambda \mathcal{Q}_{B} \geqslant \Theta_{A \rightarrow B}\left[\mathcal{N}_{A}\right]-\mathcal{M}_{B},\right. \\
& \left.\mathcal{Q}_{B} \in \operatorname{CPTP}\left(B_{0} \rightarrow B_{1}\right), \Theta \in \mathfrak{F}(A \rightarrow B)\right\} .
\end{aligned}
$$


For the case $\mathfrak{F}=$ MISC, let us start by denoting $\omega_{B}$ as the Choi matrix of $\lambda \mathcal{Q}_{B}$ and $\alpha_{A B}$ as the Choi matrix of $\Theta$, we can express $d_{\mathfrak{F}}\left(\mathcal{N}_{A} \rightarrow \mathcal{M}_{B}\right)$ as

$$
\begin{array}{ll}
d_{\mathfrak{F}}\left(\mathcal{N}_{A} \rightarrow\right. & \left.\mathcal{M}_{B}\right)=\min \lambda \\
\text { subject to : } & \text { (1) } \lambda I_{B_{0}} \geqslant \omega_{B_{0}}, \text { (2) } \omega_{B} \geqslant 0, \\
& \text { (3) } \omega_{B} \geqslant \operatorname{Tr}_{A}\left[\alpha_{A B}\left(\left(J_{A}^{\mathcal{N}}\right)^{T} \otimes I_{B}\right)\right]-J_{B}^{\mathcal{M}}, \\
& \text { (4) } \alpha_{A B} \geqslant 0, \text { (5) } \alpha_{A B_{0}}=\alpha_{A_{0} B_{0}} \otimes u_{A_{1}}, \\
& \text { (6) } \alpha_{A_{1} B_{0}}=I_{A_{1} B_{0}}, \\
\text { (7) } \operatorname{Tr}\left[\alpha_{A B} X_{A B}^{i}\right]=0 \forall i=1, \ldots, n,
\end{array}
$$

where $n \equiv|A B|(|B|-1)$ and $\left\{X_{A B}^{i}\right\}_{i=1}^{n}$ are the bases of the subspace $\mathfrak{K}_{\mathfrak{F}}$ defined in Eq. (82). Here, constraints (1-3) are due to diamond norm, constraints (4-6) follow from the requirement of $\Theta$ to be a superchannel, and constraint (7) is due to the requirement that $\Theta \in \mathfrak{F}$.

Now consider a linear map $\mathcal{L}: \mathbb{R} \oplus \operatorname{Herm}(B) \oplus$ $\operatorname{Herm}(A B) \rightarrow \operatorname{Herm}\left(B_{0}\right) \oplus \operatorname{Herm}(B) \oplus \operatorname{Herm}\left(A B_{0}\right) \oplus$ $\operatorname{Herm}\left(A_{1} B_{0}\right) \oplus^{n} \mathbb{R}$, where $\oplus^{n} \mathbb{R}$ denotes $\underbrace{\mathbb{R} \oplus \ldots \oplus \mathbb{R}}$.

Its action on a generic element $\mu=\left(\lambda, \omega_{B}^{n}, \alpha_{A B}\right)$ of $\mathbb{R} \oplus$ $\operatorname{Herm}(B) \oplus \operatorname{Herm}(A B)$ such that $\lambda \in \mathbb{R}_{+}, \omega_{B} \geqslant 0, \alpha_{A B} \geqslant 0$ is

$$
\begin{aligned}
\mathcal{L}(\mu):= & \left(\lambda I_{B_{0}}-\omega_{B_{0}}, \omega_{B}-\operatorname{Tr}\left[\alpha_{A B}\left(\left(J_{A}^{\mathcal{N}}\right)^{T} \otimes I_{B}\right)\right],\right. \\
& \alpha_{A B_{0}}-\alpha_{A_{0} B_{0}} \otimes u_{A_{1}}, \alpha_{A_{1} B_{0}}, \operatorname{Tr}\left[\alpha_{A B} X_{A B}^{1}\right], \ldots, \\
& \left.\operatorname{Tr}\left[\alpha_{A B} X_{A B}^{n}\right]\right) .
\end{aligned}
$$

Taking a generic element $v=\left(\beta_{B_{0}}, \gamma_{B}, \tau_{A B_{0}}, \zeta_{A_{1} B_{0}}\right.$, $\left.t_{1}, \ldots, t_{n}\right) \quad$ of $\quad \operatorname{Herm}\left(B_{0}\right) \oplus \operatorname{Herm}(B) \oplus \operatorname{Herm}\left(A B_{0}\right) \oplus$ $\operatorname{Herm}\left(A_{1} B_{0}\right) \oplus^{n} \mathbb{R}$ such that $\beta_{B_{0}} \geqslant 0, \gamma_{B} \geqslant 0$, we have

$$
\begin{aligned}
\mathcal{L}^{*}(v)= & \left(\operatorname{Tr}\left[\beta_{B_{0}}\right], \gamma_{B}-\beta_{B_{0}} \otimes I_{B_{1}}, \tau_{A B_{0}} \otimes I_{B_{1}}-\left(J_{A}^{\mathcal{N}}\right)^{T}\right. \\
& \otimes \gamma_{B}-\tau_{A_{0} B_{0}} \otimes u_{A_{1}} \otimes I_{B_{1}}+\tau_{A_{1} B_{0}} \otimes I_{A_{0} B_{1}} \\
& \left.+\sum_{i} t_{i} X_{A B}^{i}\right) .
\end{aligned}
$$

Following Ref. [93], the dual is given by

$$
d_{\mathfrak{F}}\left(\mathcal{N}_{A} \rightarrow \mathcal{M}_{B}\right)=\max \left\{-\operatorname{Tr}\left[J_{B}^{\mathcal{M}} \gamma_{B}\right]+\operatorname{Tr}\left[\zeta_{A_{1} B_{0}}\right]\right\}
$$

where the maximum is subject to

$$
\begin{aligned}
& \beta_{B_{0}} \otimes I_{B_{1}} \geqslant \gamma_{B} \geqslant 0,1 \geqslant \operatorname{Tr}\left[\beta_{B_{0}}\right], \\
& \zeta_{A_{1} B_{0}} \in \operatorname{Herm}\left(A_{1} B_{0}\right), \tau_{A B_{0}} \in \operatorname{Herm}\left(A B_{0}\right), t_{1}, \ldots, t_{n} \in \mathbb{R}, \\
& J_{A}^{\mathcal{N}} \otimes \gamma_{B}+\tau_{A_{0} B_{0}} \otimes u_{A_{1}} \otimes I_{B_{1}}-\tau_{A B_{0}} \otimes I_{B_{1}}-\tau_{A_{1} B_{0}} \otimes I_{A_{0} B_{1}} \\
& \quad-\sum_{i} t_{i} X_{A B}^{i} \geqslant 0 .
\end{aligned}
$$

For the case of $\mathfrak{F}=$ DISC, note that the only distinction is in the choice of basis of the subspace $\mathfrak{K}_{\mathfrak{F}}$. So, in this case, the dual is given by

$$
d_{\mathfrak{F}}\left(\mathcal{N}_{A} \rightarrow \mathcal{M}_{B}\right)=\max \left\{-\operatorname{Tr}\left[J_{B}^{\mathcal{M}} \gamma_{B}\right]+\operatorname{Tr}\left[\zeta_{A_{1} B_{0}}\right]\right\},
$$

where the maximum is subject to

$$
\begin{aligned}
& \beta_{B_{0}} \otimes I_{B_{1}} \geqslant \gamma_{B} \geqslant 0,1 \geqslant \operatorname{Tr}\left[\beta_{B_{0}}\right], \\
& \zeta_{A_{1} B_{0}} \in \operatorname{Herm}\left(A_{1} B_{0}\right), \tau_{A B_{0}} \in \operatorname{Herm}\left(A B_{0}\right), t_{1}, \ldots, t_{n} \in \mathbb{R}, \\
& J_{A}^{\mathcal{N}} \otimes \gamma_{B}+\tau_{A_{0} B_{0}} \otimes u_{A_{1}} \otimes I_{B_{1}}-\tau_{A B_{0}} \otimes I_{B_{1}}-\tau_{A_{1} B_{0}} \otimes I_{A_{0} B_{1}} \\
& \quad-\sum_{i} t_{i} Y_{A B}^{i} \geqslant 0 .
\end{aligned}
$$

Therefore, we see that $d_{\mathfrak{F}}\left(\mathcal{N}_{A} \rightarrow \mathcal{M}_{B}\right)$ is an SDP in the dynamical resource theory of quantum coherence if the free superchannels belong to MISC or DISC.

\section{APPENDIX C: UPPER BOUND ON THE LOG-ROBUSTNESS OF COHERENCE AND THE LOG-ROBUSTNESS OF QUANTUM FOURIER TRANSFORM CHANNEL AND THE MAXIMALLY COHERENT REPLACEMENT CHANNEL}

The log-robustness of a channel $\mathcal{N}_{A}$ can be expressed as

$$
\begin{aligned}
L R_{\mathfrak{C}}\left(\mathcal{N}_{A}\right) & =\log \max \left\{\operatorname{Tr}\left[\eta_{A} J_{A}^{\mathcal{N}}\right]: \mathcal{D}_{A}\left(\eta_{A}\right)=\mathcal{D}_{A_{0}}\left(\eta_{A_{0}}\right) \otimes u_{A_{1}}, \quad \mathcal{D}_{A_{1}}\left[\eta_{A_{1}}\right]=I_{A_{1}}, \quad \eta_{A} \geqslant 0\right\} \\
& =\log \max \left\{\left|A_{0} A_{1}\right| \operatorname{Tr}\left[\frac{\eta_{A}}{\left|A_{1}\right|} \frac{J_{A}^{\mathcal{N}}}{\left|A_{0}\right|}\right]: \mathcal{D}_{A}\left(\eta_{A}\right)=\mathcal{D}_{A_{0}}\left(\eta_{A_{0}}\right) \otimes u_{A_{1}}, \mathcal{D}_{A_{1}}\left[\eta_{A_{1}}\right]=I_{A_{1}}, \eta_{A} \geqslant 0\right\} .
\end{aligned}
$$

Let $\rho_{A}:=\frac{\eta_{A}}{\left|A_{1}\right|}, \sigma_{A}:=\frac{J_{\mathcal{N}}}{\left|A_{0}\right|}$, and observing that $\rho_{A}, \sigma_{A} \in \mathfrak{D}(A)$, we can rewrite the above expression as

$$
L R_{\mathfrak{C}}\left(\mathcal{N}_{A}\right)=\log \max \left\{\left|A_{0} A_{1}\right| \operatorname{Tr}\left[\rho_{A} \sigma_{A}\right]: \mathcal{D}_{A}\left(\rho_{A}\right)=\mathcal{D}_{A_{0}}\left(\rho_{A_{0}}\right) \otimes \frac{u_{A_{1}}}{\left|A_{1}\right|}, \mathcal{D}_{A_{1}}\left[\rho_{A_{1}}\right]=u_{A_{1}}, \rho_{A} \geqslant 0\right\} .
$$

Recall that the maximum of the trace of the product of two density matrices is 1 and can be obtained if the two density matrices are same and pure, i.e., $\rho_{A}=\sigma_{A}$ and $\operatorname{Tr}\left[\sigma_{A}^{2}\right]=$ $\operatorname{Tr}\left[\sigma_{A}\right]=1$. Therefore, for any channel $\mathcal{N}_{A}$,

$$
\operatorname{LR}_{\mathfrak{C}}\left(\mathcal{N}_{A}\right) \leqslant \log \left|A_{0} A_{1}\right|
$$

with $\left|A_{0}\right|=\left|A_{1}\right|=d$, the upper bound on the log-robustness of coherence becomes

$$
L R_{\mathfrak{C}}\left(\mathcal{N}_{A}\right) \leqslant \log d^{2}
$$

To achieve this upper bound of the log-robustness of coherence, we require

$$
\frac{\eta_{A}}{\left|A_{1}\right|}=\frac{J_{A}^{\mathcal{N}}}{\left|A_{0}\right|}
$$


and $\sigma_{A}=\frac{J_{A}^{\mathcal{N}}}{\left|A_{0}\right|}$ to be pure. Thus, for $\sigma_{A}$ to be pure, $\mathcal{N}_{A}$ has to be a unitary channel, i.e.,

$$
J_{A}^{\mathcal{N}}=\sum_{x, y}|x\rangle\langle y|\otimes U| x\rangle\langle y| U^{\dagger},
$$

Since for a unitary channel $J_{A}^{\mathcal{N}}=\eta_{A}\left(\left|A_{0}\right|=\left|A_{1}\right|\right.$ for a unitary channel), $J_{A}^{\mathcal{N}}$ has to follow the constraints in Eq. (C1) which can be expressed as

$$
\begin{aligned}
\mathcal{D}_{A}\left(J_{A}^{\mathcal{N}}\right) & =\mathcal{D}_{A_{0}}\left(J_{A_{0}}^{\mathcal{N}}\right) \otimes u_{A_{1}}, \\
\mathcal{D}_{A_{1}}\left[J_{A_{1}}^{\mathcal{N}}\right] & =I_{A_{1}}, \\
J_{A}^{\mathcal{N}} & \geqslant 0 .
\end{aligned}
$$

Constraint in Eq. (C10) follows from the definition of a Choi matrix of a channel and Eq. (C9) follows trivially for the Choi matrix of any unitary channel $U$. From constraint in Eq. (C8), we can find the condition on the unitary matrix to achieve the upper bound of the log-robustness of coherence of channels in the following way. First, we can write the 1.h.s. of Eq. (C8) as

$$
\begin{aligned}
\mathcal{D}_{A}\left(J_{A}^{\mathcal{N}}\right) & =\mathcal{D}_{A}\left(\sum_{x, y}|x\rangle\langle y|\otimes U| x\rangle\langle y| U^{\dagger}\right) \\
& =\sum_{x, y, i, j} \delta_{x, i} \delta_{y, j}|i\rangle\left\langle i\left|\otimes\langle j|U| x\rangle\left\langle y\left|U^{\dagger}\right| j\right\rangle\right| j\right\rangle\langle j| \\
& =\sum_{i, j}|i\rangle\left\langle i\left|\otimes\langle j|U| i\rangle\left\langle i\left|U^{\dagger}\right| j\right\rangle\right| j\right\rangle\langle j| \\
& =\sum_{i, j}\left|u_{i j}\right|^{2}|i\rangle\langle i|\otimes| j\rangle\langle j|,
\end{aligned}
$$

where $u_{i j}=\langle j|U| i\rangle$. Simplifying the r.h.s. of constraint in Eq. (C8) we get

$$
\mathcal{D}_{A_{0}}\left(J_{A_{0}}^{\mathcal{N}}\right) \otimes u_{A_{1}}=I_{A_{0}} \otimes u_{A_{1}} .
$$

Now equating Eqs. (C14) and (C15) we get

$$
\sum_{i, j}\left|u_{i j}\right|^{2}|i\rangle\langle i|\otimes| j\rangle\langle j|=I_{A_{0}} \otimes u_{A_{1}},
$$

which implies that

$$
\left|u_{i j}\right|^{2}=\frac{1}{\left|A_{1}\right|}=\frac{1}{d} \forall i, j
$$

of a unitary channel that achieves the upper bound of the logrobustness of coherence.

\section{Log-robustness of coherence of QFT channel}

The action of a quantum Fourier transform (QFT) on a basis vector is given by

$$
F_{d}|i\rangle=\frac{1}{\sqrt{d}} \sum_{k=0}^{d-1} \omega^{i k}|k\rangle,
$$

where $d$ denotes the dimension of the system the QFT is acting on and $\omega$ is the complex root of unity. Therefore, a general element of the QFT matrix can be written as

$$
\left\langle j\left|F_{d}\right| i\right\rangle=\frac{\omega^{i j}}{\sqrt{d}} .
$$

It is trivial to check that it follows the condition required to achieve the upper bound of the log-robustness of coherence, i.e.,

$$
\begin{aligned}
\left|\left\langle j\left|F_{d}\right| i\right\rangle\right|^{2} & =\left|\frac{\omega^{i j}}{\sqrt{d}}\right|^{2} \\
& =\frac{1}{d} .
\end{aligned}
$$

Therefore, the log-robustness of quantum Fourier transform channel $\left(\mathcal{N}_{A}^{F_{d}}\right)$ is

$$
\operatorname{LR}_{\mathfrak{C}}\left(\mathcal{N}_{A}^{F_{d}}\right)=\log d^{2}=2 \log d
$$

\section{Log-robustness of maximal replacement channels}

The Choi matrix of a maximal replacement channels $\mathcal{N}_{A}$ is given by

$$
J_{A}^{\mathcal{N}}=\mathrm{id} \otimes \phi_{A_{1}}^{+},
$$

where the density matrix of the maximally coherent state in dimension $\mathrm{d}$ is given by

$$
\phi_{A_{1}}^{+}=\frac{1}{d}\left(\begin{array}{ccccc}
1 & 1 & 1 & \cdots & 1 \\
1 & 1 & 1 & \cdots & 1 \\
1 & 1 & 1 & \cdots & 1 \\
\vdots & \vdots & \vdots & & \vdots \\
1 & 1 & 1 & \cdots & 1
\end{array}\right)_{d \times d} .
$$

Using Eq. (C1), we can find the log-robustness of $J_{\mathcal{A}}^{\mathcal{N}}$ as follows:

$$
\begin{aligned}
\operatorname{LR}_{\mathfrak{C}}\left(\mathcal{N}_{A}\right) & =\log \max \operatorname{Tr}\left[\eta_{A} J_{A}^{\mathcal{N}}\right] \\
& =\log \max \operatorname{Tr}\left[\eta_{A}\left(\mathrm{id}_{A_{0}} \otimes \phi_{A_{1}}^{+}\right)\right] \\
& =\log _{2} \max \operatorname{Tr}\left[\eta_{A_{1}} \phi_{A_{1}}^{+}\right],
\end{aligned}
$$

where $\eta_{A}$ follows the following conditions:

$$
\mathcal{D}_{A}\left(\eta_{A}\right)=\mathcal{D}_{A_{0}}\left(\eta_{A_{0}}\right) \otimes u_{A_{1}}, \quad \mathcal{D}_{A_{1}}\left[\eta_{A_{1}}\right]=I_{A_{1}}, \quad \eta_{A} \geqslant 0 .
$$

Equation (C27) suggests that the Choi matrix of the maximal replacement channel is the log of the max of the sum of all the elements of $\eta_{A_{1}}$ divided by $d$. And from the constraints on $\eta_{A}$, we know that the diagonal elements of $\eta_{A_{1}}$ are all 1's. Hence, the maximum value would be obtained when all the elements of $\eta_{A_{1}}$ are equal to 1 . If any off-diagonal element (and so its diagonally opposite element) are greater than 1, then the determinant of the leading principal minor will be negative which contradicts the positive semidefinite constraint imposed on $\eta_{A}$. Hence, the log-robustness of a maximally coherent replacement channel, i.e., a channel $\mathcal{N}_{A}$ that replaces any input by the maximally coherent state of dimension $d$ is given by

$$
L R_{\mathfrak{C}}\left(\mathcal{N}_{A}\right)=\log d .
$$

In conclusion, we find that the ratio between the logrobustness of coherence of QFT channel and the maximal coherent replacement channels is always 2 implying that two maximal replacement channels are required to simulate a QFT channel. One interpretation of this finding can be given by combining the resources of entanglement and coherence. 
As noted above, a distinguishing feature between the QFT and the maximal replacement channel is that the latter is entanglement-breaking. Hence, even though it can generate maximal coherence, in the process of doing so it will destroy any entanglement the primary system may have with an external one. For example, when acting on the first subsystem in the entangled state $\sqrt{1 / 2}(|00\rangle+|11\rangle)$, the qubit replacement channel will yield $\rho_{1}=\phi_{2}^{+} \otimes I / 2$, while the QFT will yield $\left|\psi_{2}\right\rangle=\sqrt{1 / 2}(|0+\rangle+|1-\rangle)$, where $| \pm\rangle=\sqrt{1 / 2}(|0\rangle \pm|1\rangle)$.

A combined resource theory of entanglement and coherence was studied in Ref. [43]. In particular, the asymptotic convertibility of states using local incoherent operations was considered, and for a given state $\rho^{A B}$, one can define the optimal rate sum $R_{C}+R_{E}$ of coherent bits $\left(R_{C}\right)$ and entangled bits $\left(R_{E}\right)$ needed to asymptotically prepare the state $\rho^{A B}$. It turns out that state $\left|\psi_{2}\right\rangle$ has twice the resource cost as state $\rho_{1}$, when resource cost is measured in terms of the smallest rate sum $R_{C}+R_{E}$. Here, $R_{C}$ and $R_{E}$ are the asymptotic rates of coherence and entanglement used to build the state. Hence, the greater resource power of the QFT versus the replacement channel becomes operationally manifest in this way.
[1] W. H. Zurek, Decoherence, einselection, and the quantum origins of the classical, Rev. Mod. Phys. 75, 715 (2003).

[2] M. Schlosshauer, Decoherence, the measurement problem, and interpretations of quantum mechanics, Rev. Mod. Phys. 76, 1267 (2005).

[3] D. A. Lidar and K. Birgitta Whaley, Decoherence-free subspaces and subsystems, in Irreversible Quantum Dynamics, edited by Fabio Benatti and Roberto Floreanini (Springer, Berlin/Heidelberg, 2003), pp. 83-120.

[4] D. M. Bacon, Decoherence, control, and symmetry in quantum computers, Ph.D. thesis, University of California, Berkeley, 2001.

[5] R. Horodecki, P. Horodecki, M. Horodecki, and K. Horodecki, Quantum entanglement, Rev. Mod. Phys. 81, 865 (2009).

[6] M. B. Plenio and S. Virmani, An introduction to entanglement measures, Quantum Inf. Comput. 7, 1 (2007).

[7] S. D. Bartlett, T. Rudolph, and R. W. Spekkens, Reference frames, superselection rules, and quantum information, Rev. Mod. Phys. 79, 555 (2007).

[8] J. Goold, M. Huber, A. Riera, L. Rio, and P. Skrzypczyk, The role of quantum information in thermodynamics-A topical review, J. Phys. A: Math. Theor. 49, 143001 (2016).

[9] G. Gour, M. P. Muller, V. Narasimhachar, R. W. Spekkens, and N. Y. Halpern, The resource theory of informational nonequilibrium in thermodynamics, Phys. Rep. 583, 1 (2015).

[10] N. Brunner, D. Cavalcanti, S. Pironio, V. Scarani, and S. Wehner, Bell nonlocality, Rev. Mod. Phys. 86, 419 (2014).

[11] C. Weedbrook, S. Pirandola, R. García-Patrón, N. J. Cerf, T. C. Ralph, J. H. Shapiro, and S. Lloyd, Gaussian quantum information, Rev. Mod. Phys. 84, 621 (2012).

[12] Á. Rivas, S. F. Huelga, and M. B. Plenio, Quantum nonmarkovianity: characterization, quantification and detection, Rep. Prog. Phys. 77, 094001 (2014).

[13] G. Adesso, T. R. Bromley, and M. Cianciaruso, Measures and applications of quantum correlations, J. Phys. A: Math. Theor. 49, 473001 (2016).

[14] K. Modi, A. Brodutch, H. Cable, T. Paterek, and V. Vedral, The classical-quantum boundary for correlations: Discord and related measures, Rev. Mod. Phys. 84, 1655 (2012).

[15] E. Chitambar and G. Gour, Quantum resource theories, Rev. Mod. Phys. 91, 025001 (2019).

[16] W. H. Zurek, Decoherence and the transition from quantum to classical, Phys. Today 44(10), 36 (1991).

[17] W. H. Zurek, Decoherence and the Transition from Quantum to Classical-Revisited, in Quantum Decoherence: Poincaré
Seminar 2005, edited by Bertrand Duplantier, Jean-Michel Raimond, and Vincent Rivasseau (Birkhäuser, Basel, 2007), pp. $1-31$.

[18] C. H. Bennett, G. Brassard, S. Popescu, B. Schumacher, J. A Smolin, and W. K. Wootters, Purification of Noisy Entanglement and Faithful Teleportation via Noisy Channels, Phys. Rev. Lett. 76, 722 (1996).

[19] C. H. Bennett, D. P. DiVincenzo, J. A. Smolin, and W. K. Wootters, Mixed-state entanglement and quantum error correction, Phys. Rev. A 54, 3824 (1996).

[20] H.-K. Lo and S. Popescu, Concentrating entanglement by local actions: Beyond mean values, Phys. Rev. A 63, 022301 (2001).

[21] J. Aberg, Quantifying superposition, arXiv:quant-ph/0612146.

[22] T. Baumgratz, M. Cramer, and M. B. Plenio, Quantifying Coherence, Phys. Rev. Lett. 113, 140401 (2014).

[23] E. Chitambar and G. Gour, Critical Examination of Incoherent Operations and a Physically Consistent Resource Theory of Quantum Coherence, Phys. Rev. Lett. 117, 030401 (2016).

[24] E. Chitambar and G. Gour, Comparison of incoherent operations and measures of coherence, Phys. Rev. A 94, 052336 (2016).

[25] E. Chitambar and G. Gour, Erratum: Comparison of incoherent operations and measures of coherence [Phys. Rev. A 94, 052336 (2016)], Phys. Rev. A 95, 019902(E) (2017).

[26] I. Marvian and R. W. Spekkens, How to quantify coherence: Distinguishing speakable and unspeakable notions, Phys. Rev. A 94, 052324 (2016).

[27] T. Theurer, D. Egloff, L. Zhang, and M. B. Plenio, Quantifying Operations with an Application to Coherence, Phys. Rev. Lett. 122, 190405 (2019).

[28] A. Streltsov, G. Adesso, and M. B. Plenio, Colloquium: Quantum coherence as a resource, Rev. Mod. Phys. 89, 041003 (2017).

[29] K. B. Dana, M. García Díaz, M. Mejatty, and A. Winter, Resource theory of coherence: Beyond states, Phys. Rev. A 95, 062327 (2017).

[30] M. G. Díaz, K. Fang, X. Wang, M. Rosati, M. Skotiniotis, J. Calsamiglia, and A. Winter, Using and reusing coherence to realize quantum processes, Quantum 2, 100 (2018).

[31] A. Mani and V. Karimipour, Cohering and decohering power of quantum channels, Phys. Rev. A 92, 032331 (2015).

[32] María García-Díaz, D. Egloff, and M. B. Plenio, A note on coherence power of n-dimensional unitary operators, Quantum Inf. Comput. 16, 12821294 (2016). 
[33] P. Zanardi, G. Styliaris, and L. Campos Venuti, Measures of coherence-generating power for quantum unital operations, Phys. Rev. A 95, 052307 (2017).

[34] M. Takahashi and E. Chitambar, Comparing coherence and entanglement under resource non-generating unitary transformations, J. Phys. A: Math. Theor. 51, 414003 (2018).

[35] Y. Liu and X. Yuan, Operational resource theory of quantum channels, Phys. Rev. Research 2, 012035 (2020).

[36] Z.-W. Liu, X. Hu, and S. Lloyd, Resource Destroying Maps, Phys. Rev. Lett. 118, 060502 (2017).

[37] D. Rosset, F. Buscemi, and Y.-C. Liang, Resource Theory of Quantum Memories and Their Faithful Verification with Minimal Assumptions, Phys. Rev. X 8, 021033 (2018).

[38] T. Simnacher, N. Wyderka, C. Spee, X.-D. Yu, and O. Gühne, Certifying quantum memories with coherence, Phys. Rev. A 99, 062319 (2019).

[39] X. Yuan, Y. Liu, Q. Zhao, B. Regula, J. Thompson, and M. Gu, Robustness of quantum memories: An operational resourcetheoretic approach, arXiv:1907.02521.

[40] Note that it is important to consider some specified amount of time or distance, or else one might argue that the preservation of coherence for a longer duration of time (or for a longer distance) is more resourceful than for lesser amount of time (or shorter distance). Since this is not the primary problem addressed in this work, we leave it as an open question to be explored further.

[41] S. Wehner, D. Elkouss, and R. Hanson, Quantum internet: A vision for the road ahead, Science 362, eaam9288 (2018)

[42] S. Pirandola, R. Laurenza, C. Ottaviani, and L. Banchi, Fundamental limits of repeaterless quantum communications, Nat. Commun. 8, 15043 (2017).

[43] E. Chitambar and M.-H. Hsieh, Relating the Resource Theories of Entanglement and Quantum Coherence, Phys. Rev. Lett. 117, 020402 (2016).

[44] G. Gour, Comparison of quantum channels by superchannels, IEEE Trans. Inf. Theory 65, 5880 (2019).

[45] G. Gour and A. Winter, How to Quantify a Dynamical Resource? Phys. Rev. Lett. 123, 150401 (2019).

[46] G. Gour and M. M. Wilde, Entropy of a quantum channel, arXiv: 1808.06980.

[47] L. Li, K. Bu, and Z.-W. Liu, Quantifying the resource content of quantum channels: An operational approach, Phys. Rev. A 101, 022335 (2020).

[48] M. Berta, C. Hirche, E. Kaur, and M. M. Wilde, Amortized channel divergence for asymptotic quantum channel discrimination, arXiv: 1808.01498 .

[49] X. Wang and M. M. Wilde, Exact entanglement cost of quantum states and channels under ppt-preserving operations, arXiv:1809.09592.

[50] P. Faist, M. Berta, and F. Brandão, Thermodynamic Capacity of Quantum Processes, Phys. Rev. Lett. 122, 200601 (2019).

[51] G. Gour and C. M. Scandolo, The entanglement of a bipartite channel, arXiv:1907.02552.

[52] B. Coecke, T. Fritz, and R. W. Spekkens, A mathematical theory of resources, Quantum Inf. Comput. 250, 59 (2016).

[53] Z.-W. Liu and A. Winter, Resource theories of quantum channels and the universal role of resource erasure, arXiv:1904.04201.

[54] E. Kaur and M. M Wilde, Amortized entanglement of a quantum channel and approximately teleportation-simulable channels, J. Phys. A: Math. Theor. 51, 035303 (2017).
[55] G. Chiribella, G. M. DAriano, and P. Perinotti, Transforming quantum operations: Quantum supermaps, Europhys. Lett. 83, 30004 (2008)

[56] P. Perinotti, Causal structures and the classification of higher order quantum computations, in Time in Physics, edited by Renato Renner and Sandra Stupar (Springer International Publishing, Cham, 2017), pp. 103-127.

[57] G. Chiribella, G. M. D'Ariano, and P. Perinotti, Theoretical framework for quantum networks, Phys. Rev. A 80, 022339 (2009).

[58] G. Chiribella, G. M. D’Ariano, P. Perinotti, and B. Valiron, Quantum computations without definite causal structure, Phys. Rev. A 88, 022318 (2013).

[59] A. Bisio and P. Perinotti, Theoretical framework for higherorder quantum theory, Proc. R. Soc. London A 475, 20180706 (2019).

[60] J. Burniston, M. Grabowecky, C. M. Scandolo, G. Chiribella, and G. Gour, Necessary and sufficient conditions on measurements of quantum channels, Proc. R. Soc. A. 476, 20190832 (2020).

[61] T. R. Bromley, M. Cianciaruso, and G. Adesso, Frozen Quantum Coherence, Phys. Rev. Lett. 114, 210401 (2015).

[62] A. Streltsov, U. Singh, H. S. Dhar, M. N. Bera, and G. Adesso, Measuring Quantum Coherence with Entanglement, Phys. Rev. Lett. 115, 020403 (2015).

[63] A. Winter and D. Yang, Operational resource theory of coherence, Phys. Rev. Lett. 116, 120404 (2016).

[64] B. Yadin, J. Ma, D. Girolami, M. Gu, and V. Vedral, Quantum Processes Which Do Not use Coherence, Phys. Rev. X 6, 041028 (2016).

[65] G. Chiribella and Y. Yang, Optimal quantum operations at zero energy cost, Phys. Rev. A 96, 022327 (2017).

[66] J. I. de Vicente and A. Streltsov, Genuine quantum coherence, J. Phys. A: Math. Theor. 50, 045301 (2016).

[67] K. Fang, O. Fawzi, R. Renner, and D. Sutter, A Chain Rule for the Quantum Relative Entropy, Phys. Rev. Lett. 124, 100501 (2020).

[68] K. Fang, X. Wang, M. Tomamichel, and M. Berta, Quantum channel simulation and the channel's smooth max-information, in 2018 IEEE International Symposium on Information Theory (ISIT) (IEEE, Piscataway, NJ, 2018), pp. 2326-2330.

[69] S. Bauml, S. Das, X. Wang, and M. M. Wilde, Resource theory of entanglement for bipartite quantum channels, arXiv:1907.04181.

[70] L. Gurvits, Classical deterministic complexity of edmonds' problem and quantum entanglement, in Proceedings of the 35th Annual ACM Symposium on Theory of Computing, STOC'03 (ACM, New York, NY, 2003), pp. 10-19.

[71] A. Rényi, On measures of entropy and information, in Proceedings of the 4th Berkeley Symposium on Mathematical Statistics and Probability, Volume 1: Contributions to the Theory of Statistics (University of California Press, Berkeley, CA, 1961), pp. 547-561.

[72] M. M. Wilde, A. Winter, and D. Yang, Strong converse for the classical capacity of entanglement-breaking and Hadamard channels via a sandwiched Rényi relative entropy, Commun. Math. Phys. 331, 593 (2014).

[73] M. Müller-Lennert, F. Dupuis, O. Szehr, S. Fehr, and M. Tomamichel, On quantum Rényi entropies: A new 
generalization and some properties, J. Math. Phys. 54, 122203 (2013).

[74] N. Datta and F. Leditzky, A limit of the quantum rényi divergence, J. Phys. A: Math. Theor. 47, 045304 (2014).

[75] R. L. Frank and E. H. Lieb, Monotonicity of a relative Rényi entropy, J. Math. Phys. 54, 122201 (2013).

[76] F. Hiai, M. Mosonyi, D. Petz, and C. Beny, Quantum fdivergences and error correction, Rev. Math. Phys. 23, 691 (2011).

[77] F. Hiai and M. Mosonyi, Different quantum f-divergences and the reversibility of quantum operations, Rev. Math. Phys. 29, 1750023 (2017).

[78] M. M. Wilde, Optimized quantum f-divergences and data processing, J. Phys. A: Math. Theor. 51, 374002 (2018).

[79] K. M. R. Audenaert and N. Datta, $\alpha$-z-Rényi relative entropies, J. Math. Phys. 56, 022202 (2015).

[80] T. Cooney, M. Mosonyi, and M. M. Wilde, Strong converse exponents for a quantum channel discrimination problem and quantum-feedback-assisted communication, Commun. Math. Phys. 344, 797 (2016).

[81] F. Leditzky, E. Kaur, N. Datta, and M. M. Wilde, Approaches for approximate additivity of the holevo information of quantum channels, Phys. Rev. A 97, 012332 (2018).

[82] N. Datta, Max-relative entropy of entanglement, alias log robustness, Int. J. Quantum. Inform. 07, 475 (2009).
[83] N. Datta, Min- and max-relative entropies and a new entanglement monotone, IEEE Trans. Inf. Theory 55, 2816 (2009).

[84] F. Buscemi and N. Datta, The quantum capacity of channels with arbitrarily correlated noise, IEEE Trans. Inf. Theory 56, 1447 (2010).

[85] F. G. S. L. Brandao and N. Datta, One-shot rates for entanglement manipulation under non-entangling maps, IEEE Trans. Inf. Theory 57, 1754 (2011).

[86] K. Bu, U. Singh, S.-M. Fei, A. K. Pati, and J. Wu, Maximum Relative Entropy of Coherence: An Operational Coherence Measure, Phys. Rev. Lett. 119, 150405 (2017).

[87] J. Watrous, Semidefinite programs for completely bounded norms, Theory Comput. 5, 217 (2009).

[88] https://github.com/gaurav-iiser/Resource_Theory_of_ Dynamical_Coherence.

[89] G. Gour, I. Marvian, and R. W. Spekkens, Measuring the quality of a quantum reference frame: The relative entropy of frameness, Phys. Rev. A 80, 012307 (2009).

[90] B. Regula, K. Fang, X. Wang, and G. Adesso, One-Shot Coherence Distillation, Phys. Rev. Lett. 121, 010401 (2018).

[91] Q. Zhao, Y. Liu, X. Yuan, E. Chitambar, and A. Winter, Oneshot coherence distillation: Towards completing the picture, IEEE Trans. Inf. Theory 65, 6441 (2019).

[92] C.-Y. Hsieh, Resource preservability, Quantum 4, 244 (2020).

[93] A. Barvinok, A Course in Convexity, Vol. 54 (American Mathematical Society, Providence, RI, 2002) 